\title{
Ab initio studies of anisotropic magnetism in uranium and cerium monopnictides and monochalcogenides
}

\author{
Eric Mason Collins \\ West Virginia University
}

Follow this and additional works at: https://researchrepository.wvu.edu/etd

\section{Recommended Citation}

Collins, Eric Mason, "Ab initio studies of anisotropic magnetism in uranium and cerium monopnictides and monochalcogenides" (2000). Graduate Theses, Dissertations, and Problem Reports. 3190.

https://researchrepository.wvu.edu/etd/3190

This Dissertation is protected by copyright and/or related rights. It has been brought to you by the The Research Repository @ WVU with permission from the rights-holder(s). You are free to use this Dissertation in any way that is permitted by the copyright and related rights legislation that applies to your use. For other uses you must obtain permission from the rights-holder(s) directly, unless additional rights are indicated by a Creative Commons license in the record and/ or on the work itself. This Dissertation has been accepted for inclusion in WVU Graduate Theses, Dissertations, and Problem Reports collection by an authorized administrator of The Research Repository @ WVU.

For more information, please contact researchrepository@mail.wvu.edu. 
$A b$ Initio Studies of Anisotropic Magnetism in Uranium and Cerium Monopnictides and Monochalcogenides

Eric Mason Collins

Dissertation submitted to the

Eberly College of Arts and Sciences

at West Virginia University

in partial fulfillment of the requirements

for the degree of

Doctor of Philosophy

in

Physics

Bernard R. Cooper, Ph.D., Chair

Nicholas Kioussis, Ph.D.

Mohindar S. Seehra, Ph.D.

H. Arthur Weldon, Ph.D.

Martin V. Ferer, Ph.D.

Keh-Minn Chang, Ph.D.

Department of Physics

Morgantown, West Virginia

2000

Keywords: Magnetism, Uranium, Cerium

Copyright 2000 Eric M. Collins 


\section{ABSTRACT \\ Ab Initio Studies of Anisotropic Magnetism in Uranium and Cerium Monopnictides and Monochalcogenides \\ Eric Mason Collins}

We have applied two $a b$ initio based methods to investigate the origin in the electronic structure of the unusual magnetic behavior of the cerium and uranium monopnictides and monochalcogenides. First, we have carried out spin-polarized electronic structure calculations, based on the full potential linear muffin tin (FPLMTO) method, with spin polarization (orbital polarization only via spin- orbit coupling) and also with orbital polarization correction. Second, we have carried out ab initio based calculations synthesizing 1) a phenomenological theory of orbitally driven magnetism based on the Anderson and Kondo lattice model which incorporates explicitly the hybridization induced and the Coulomb exchange interactions on an equal footing, and 2) FPLMTO electronic structure calculations allowing a first principles evaluation of all the parameters entering the model Hamiltonian. For the cerium compounds, we also include the crystal field interactions on an equal footing with the hybridization and Coulomb exchange interactions with a scaling determined by experiment. The results for the uranium compound calculations show that both methods are limited to the extremes to which they are best suited. The pure band structure calculations provide the best agreement for the lighter uranium compounds, while the model hamiltonian approach provides better agreement for the heavier uranium compounds. In the case of the cerium compounds, while the pure FPLMTO calculations yield values for the magnetic moment in agreement with experiment for the lighter cerium chalcogenides, they fail to give, even qualitatively, the magnetic properties for all other systems. On the other hand, the $a b$ initio based model Hamiltonian calculations reveal for the first time the interplay of hybridization, Coulomb exchange, and crystal field interactions across the cerium series, and give results for the low-temperature moment and ordering temperature in excellent agreement with experiment, for the full range of both types of cerium compounds. 
Dedicated in loving memory to

Alfretta Kerns 


\section{ACKNOWLEDGEMENTS}

First, I would like to thank my Lord and Savior Jesus Christ, for creating the universe, giving it order, giving these compounds interesting properties, allowing me to have the ability and the opportunity to study these compounds, and for those people who made this work possible.

I would like to thank my academic advisor, Dr. Barry Cooper, for introducing me to this field of research, and for wise counsel, and for motivation to constantly move forward. Huge thanks go to my research advisor (and boss), Dr. Nick Kioussis, who's heavy hand taught me to always look to understand the physics behind the numbers, and for all of your invaluable contributions to this work. I would also like to thank Dr. Say-Peng Lim for getting me started on research in this field, and for guidance on how to approach various snags and problems encountered on the way. I'd also like to thank my dissertation committee: Dr. Seehra, Dr. Ferer, Dr. Weldon, and Dr. Chang, for all of your comments and input on this work, and for your patience in scheduling and giving exams.

Thanks to the office staff at West Virginia University: Dr. Halliburton, Sherry, Siobhan, and Sharon. For holding things up on your end. And to Dr. Herzog, Linda, Margie, and Carole at Cal State Northridge, for helping me to hold things up on my end. Be sure to thank any "gophers" for me as well.

To other folks who have given me time and effort: just about every professor at WVU. To Kevin and Paul, wherever you find yourselves, thanks for motivating me through my first years back there. To the Gibbies crowd (Greg, Sherry, Jack, and Richard) when you read this have one for me.

Personal thanks go to: Brian, Natalie, and Monte for providing housing for me in Morgantown. To James, Bruce, and Jon for putting up with a physicist as a roommate. To Alan, Steve, and Scott for a good video game or two. To John, Tom, and Karin for all the personal advice and encouragement. To my best friend, Jerry, for being there. And to my mom....cant say enough about my mom...and my family.

Thanks to all those plus many others that I simply don't have the space in my dissertation to thank. 


\section{Contents}

1 INTRODUCTION 1

1.1 INFORMATION FROM EXPERIMENTS ON CERIUM COMPOUNDS 6

1.2 INFORMATION FROM EXPERIMENTS ON URANIUM COMPOUNDS 8

1.3 MOTIVATION AND APPROACH .............. 10

2 METHODS OF SOLUTION 14

2.1 THE FPLMTO METHOD . . . . . . . . . . . . . . 16

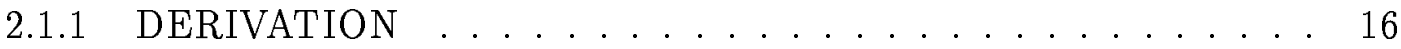

2.1 .2 ORBITAL POLARIZATION ............. 20

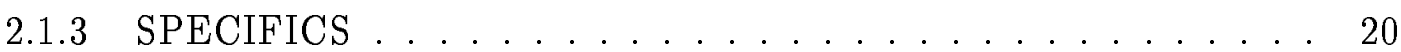

2.2 THE MODEL HAMILTONIAN METHOD . . . . . . . . . . . . . 22

2.2.1 DERIVATION OF THE MODEL HAMILTONIAN . . . . . . . 22

2.2.2 AB INITIO CALCULATION OF PARAMETERS . . . . . . . 26

2.2.3 THE TWO-ION INTERACTION . . . . . . . . . . . 35

2.2.4 THE MEAN FIELD CALCULATION . . . . . . . . . . . 39

2.3 THEORETICAL UNCERTAINTIES . . . . . . . . . . . . . . 43

2.3.1 UNCERTAINTY IN FPLMTO METHOD . . . . . . . . . . 43

2.3.2 UNCERTAINTY IN THE MODEL HAMILTONIAN METHOD . 44

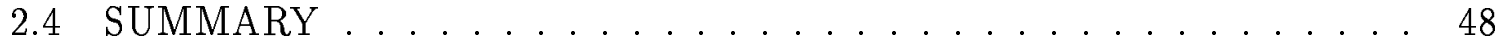

3 RESULTS ON CERIUM COMPOUNDS $\quad 51$

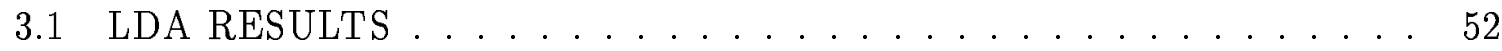

3.2 MODEL HAMILTONIAN RESULTS . . . . . . . . . . . . . . 66 


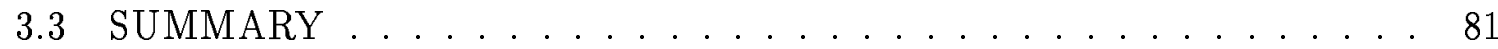

4 RESULTS ON URANIUM COMPOUNDS $\quad 86$

4.1 LDA RESULTS . . . . . . . . . . . . . . . 87

4.2 MODEL HAMILTONIAN RESULTS . . . . . . . . . . . . 92

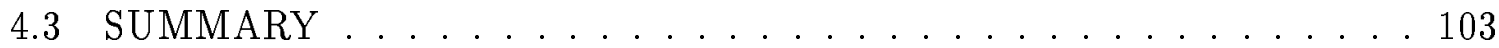

4.4 FURTHER STUDIES . . . . . . . . . . . . . . . 104

4.4.1 LDA+U CALCULATIONS (MLDA) . . . . . . . . . 104

4.4.2 CRYSTAL FIELD TERM (MHMT) . . . . . . . . . 105

4.4.3 CHANGING THE MODEL .............. 106

5 CONCLUSIONS 109 


\section{List of Figures}

1.1 Rock-salt structure found in the uranium and cerium pnictides and chalcogenides studied. The dark atoms are cation ( $\mathrm{Ce}$ or $\mathrm{U}$ ) sites and the light atoms indicate anion (pnictogen or chalcogen) sites. Also indicated are two directions the $<001>$ along the cube edge and the $<111>$ body

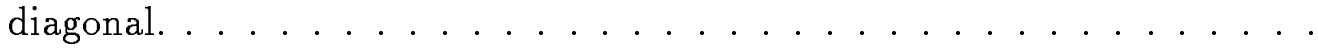

3.1 Spin magnetization density $\rho_{\downarrow}-\rho_{\uparrow}$ for $\mathrm{CeP}$ in the plane parallel to the spin polarization direction $<001>$. Solid lines indicate higher spin down density, dashed lines indicate higher spin up density. . . . . . . . . . 53

3.2 Spin magnetization density $\rho_{\downarrow}-\rho_{\uparrow}$ for CeP in the plane perpendicular to the spin polarization direction $<001>$. Solid lines indicate higher spin down density, dashed lines indicate higher spin up density. . . . . . . .

3.3 Spin magnetization density $\rho_{\downarrow}-\rho_{\uparrow}$ for $\mathrm{CeP}$ in the plane parallel to the spin polarization direction $<111>$. Solid lines indicate higher spin down density, dashed lines indicate higher spin up density. . . . . . . . . .

3.4 Spin magnetization density $\rho_{\downarrow}-\rho_{\uparrow}$ for $\mathrm{CeP}$ in the plane perpendicular to the spin polarization direction $<111>$. Solid lines indicate higher spin down density, dashed lines indicate higher spin up density. . . . . . . .

3.5 Cerium Phosphide $f$-projected density of states for spin up (shaded) and spin down (clear) electrons for the converged FPLMTO spin polarized potential without orbital polarization. The Fermi energy is marked on the graph and is given in Rydbergs. . . . . . . . . . . . . . 
3.6 Cerium Phosphide $f$-projected density of states for spin up (shaded) and spin down (clear) electrons for the converged FPLMTO spin polarized potential with orbital polarization correction. The Fermi energy is marked on the graph and is given in Rydbergs. . . . . . . . . . . .

3.7 Cerium Sulfide $f$-projected density of states for spin up (shaded) and spin down (clear) electrons for the converged FPLMTO spin polarized potential without orbital polarization. The Fermi energy is marked on the graph and is given in Rydbergs. . . . . . . . . . . . . .

3.8 Cerium Sulfide $f$-projected density of states for spin up (shaded) and spin down (clear) electrons for the converged FPLMTO spin polarized potential with orbital polarization correction. The Fermi energy is marked on the graph and is given in Rydbergs. . . . . . . . . . . .

3.9 Cerium Chalcogenide density of non-f states computed with the $f$ electrons being placed into the "core" and not allowed to hybridize with the non-f states. Densities are given in states/Ry. Fermi energies are indicated with

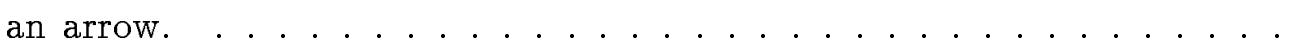

3.10 Cerium Pnictide density of non-f states computed with the $f$ electrons being placed into the "core" and not allowed to hybridize with the non-f states. Densities are given in states/Ry. Fermi energies are indicated with

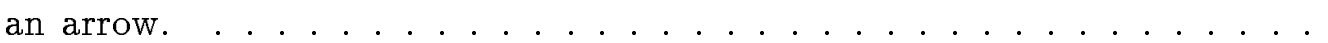

3.11 Ordered moment versus temperature calculated from the mean field hamiltonian for $\mathrm{CeSb}$ for the lowest energy $\uparrow \uparrow \downarrow \downarrow$ configuration. Shown are both graphs calculated without and with the crystal field term $(\mathrm{CF})$ taken into account. Indicated on the graph are the experimental results for the zero-temperature moment $2.06 \mu_{B}$ and the Néel temperature $17 \mathrm{~K}$.

3.12 Zero-temperature ordered moment versus the crystal field splitting (CF) term entering the model hamiltonian for cerium phosphide the ordered moment are given in $\mu_{B}$. Three regions are indicated on the graph, the exchange dominated, the transition region, and the crystal field dominated. 77 
3.13 Energy (in Kelvin units) versus temperature (in Kelvin units) for the paramagnetic (PARA), AFP $(\uparrow \downarrow 0)$, type-I $(\uparrow \downarrow)$ and type-IA $(\uparrow \uparrow \downarrow \downarrow)$ configurations for $\mathrm{CeSb}$ calculated from the mean field hamiltonian with only the exchange interactions. . . . . . . . . . . . . .

3.14 Energy (in Kelvin units) versus temperature (in Kelvin units) for the paramagnetic (PARA), AFP ( $\downarrow$ ) , type-I $(\uparrow \downarrow)$ and type-IA $(\uparrow \uparrow \downarrow \downarrow)$ configurations for $\mathrm{CeSb}$ calculated from the total mean field hamiltonian including all three pertinent interactions, (the hybridization, the Coulomb exchange and the crystal field). . . . . . . . . . . . . . 80

4.1 Uranium Sulfide $f$-projected density of states for spin up (shaded) and spin down (clear) electrons for the converged FPLMTO spin polarized potential without orbital polarization. The Fermi energy is marked on the graph and is given in Rydbergs. . . . . . . . . . . . . .

4.2 Uranium Sulfide $f$-projected density of states for spin up (shaded) and spin down (clear) electrons for the converged FPLMTO spin polarized potential with orbital polarization correction. The Fermi energy is marked on the graph and is given in Rydbergs. . . . . . . . . . . .

4.3 Uranium Sulfide density of states. The top panel shows the density of unhybridized $f$ states and the density of the unhybridized non- $f$ states is shown in the middle panel. The bottom panel shows the fully hybridized total density of states. . . . . . . . . . . . . . 93

4.4 Uranium Sulfide bonding charge density, $\rho_{\text {bond }}$. The uranium atom sites are in the center and on the corners, the sulfur sites are on the sides. . . 96

4.5 Uranium Telluride bonding charge density, $\rho_{\text {bond }}$. The uranium atom sites are in the center and on the corners, the tellurium sites are on the sides.

4.6 Uranium Phosphide bonding charge density, $\rho_{\text {bond }}$. The uranium atom sites are in the center and on the corners, the phosphorus sites are on the

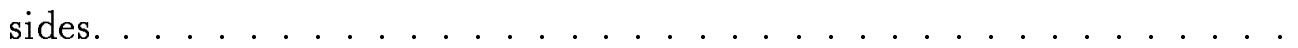




\section{List of Tables}

1.1 First principle calculated values of the ratio of Coulomb repulsion to resonance width, $U / \Gamma$, characteristic of the correlation between $f$ electrons in the uranium and cerium compounds studied. The quantities listed are

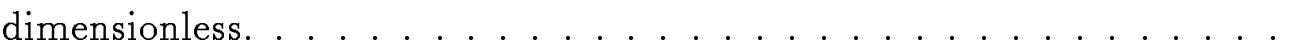

1.2 Summary of the experimental results for the various magnetic properties of the cerium compounds. Listed are the lattice constant $(a)$, the zero temperature ordered moment $\left(\mu_{0}\right)$, the ordering temperature $\left(T_{N}\right)$, the crystal field splitting $\left(\Delta_{C F}\right)$, and the direction of anisotropy. . . . . .

1.3 Experimental results for the magnetic properties of the uranium compounds studied. Listed are the lattice constant $(a)$, the zero-temperature ordered moment $\left(\mu_{0}\right)$, the ordering temperature $\left(T_{\text {ord }}\right)$, and the direction of anisotropy $\hat{M}$ with the magnetic ordering. The ordering temperature is the Curie temperature for the chalcogenides, and the Néel temperature

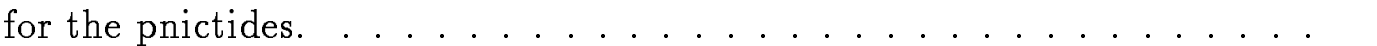

2.1 Ligand basis states for the pure FPLMTO electronic structure calculations. 21

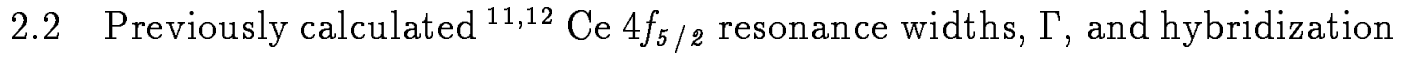
potentials, $\nu\left(\kappa_{F}\right)$, in $\mathrm{mRy}$ calculated with equations (2.32) and (2.31) respectively and used in the calculation of the hybridization from the "core" point of view. . . . . . . . . . . . . . . . . 29

2.3 Experimental values of the crystal field splitting for the cerium compounds given in Kelvin units. . . . . . . . . . . . . . . . . . . . 42 
2.4 Characteristic matrix elements of the $10 \times 10$ two-ion interaction matrix $\left(E_{m m^{\prime}}^{m^{\prime} m}(R)\right)$ of the $f^{3}$ multiplet for uranium sulfide. Values for the hybridization induced $\left(E_{V^{4}}\right)$, cross terms $\left(E_{V^{2} J}\right)$, and pure Coulomb exchange $\left(E_{J^{2}}\right)$ interactions are listed for the $m=m^{\prime}=\frac{5}{2}$ elements for each of the first, second, and third nearest neighbors in units of K. . . . . . 45

3.1 Final self-consistent values of the $f$ state Racah parameters for the compounds studied in units of mRy. . . . . . . . . . . . . . 57

3.2 Values of the calculated and experimental ${ }^{1-4}$ magnetic moments for the cerium chalcogenides and pnictides in units of $\mu_{B}$. Listed are the FPLMTO values for the spin moment $\mu_{S}$, the orbital moment $\mu_{L}$, and total moment $\mu$, for the spin polarized only calculation and for the calculation with spin polarization and orbital polarization correction. . . . . .

3.3 Values of the calculated magnetic moments for the cerium chalcogenides and pnictides in units of $\mu_{B}$. Listed are the warped muffin-tin LMTO values for the spin moment $\mu_{S}$, the orbital moment $\mu_{L}$, and total moment $\mu$ for the calculation with spin polarization and orbital polarization correction. For comparison we have also included the results for the total magnetic moment from the full potential calculation (FULL) and experimental results $(\mathrm{EXPT})^{1-4} \ldots \ldots \ldots \ldots$. . . . . . . . 64

3.4 Energy differences obtained from the FPLMTO spin-polarized with orbital polarization correction between polarization along the $\langle 001\rangle$ direction and the $\langle 111\rangle$ direction. Values obtained from both the warped muffin-tin and full potential calculations are given. Positive values indicate polarization along the $\langle 001\rangle$ direction. Also listed are the experimental easy-axis directions. Values are given in mRy. . . . . . . . . . . 64

3.5 Summary of the FPLMTO results for the Fermi energy, $E_{F}$, the Ce $4 f$ core resonance energy $E_{4 f}$ and the total density of states $N\left(E_{F}\right)$ at $E_{F}$. $E_{F}$ and $E_{4 f}$ are given in Ry, the DOS are in states/Ry. 
3.6 Sample calculation of the calculation of the energy difference, $E_{f}-E_{F}$ and the on-site coulomb repulsion, $U$, for CeS. Listed are the number of $f$ electrons placed at the central cerium site, $n_{f}$, the band calculated Fermi energy $E_{F}^{n}$, the core resonance band calculated $f$ state energy $E_{f}^{n}$, and the energy difference for each number of central site $f$ electrons, $\mathcal{E}^{n}$. . . . 67

3.7 Results from the supercell calculations for the energy difference $E_{f}-E_{F}$ and the on-site coulomb repulsion $U$ for the cerium compounds which appear in the model hamiltonian. All units are in eV. . . . . . . . . 70

3.8 Listed below are characteristic matrix elements of the 6 X6 two-ion interaction matrix $\left(E_{m_{a} m_{a}^{\prime}}^{m_{b}^{\prime}}\left(\mathbf{R}_{\mathbf{b}}-\mathbf{R}_{\mathbf{a}}\right)\right)$ of the $f$ electrons for a cross section of the cerium compounds. Values of the hybridization induced $\left(E_{V^{4}}\right)$, cross terms $\left(E_{V^{2} J}\right)$, and pure Coulomb exchange $\left(E_{J^{2}}\right)$ interactions are listed for the first, second, and third nearest cerium neighbors in units of $\mathrm{K}$. The listed elements are the $m=m^{\prime}= \pm \frac{1}{2}$. . . . . . . . . . . 71

3.9 Matrix elements from the total two ion interaction $E_{m_{a} m_{a}^{\prime}}^{m_{b}^{\prime}}\left(\mathbf{R}_{\mathbf{b}}-\mathbf{R}_{\mathbf{a}}\right)=$ $E_{V^{4}}+E_{V^{2} J}+E_{J^{2}}$ where $m= \pm \frac{1}{2}$ and $m^{\prime}= \pm \frac{1}{2}$ for the first and second nearest neighbors. . . . . . . . . . . . . . .

3.10 Calculated (from the model hamiltonian) values of the zero temperature ordered moments $\left(\mu_{0}\right)$ and ordering temperatures, Néel temperature $\left(T_{N}\right)$ both without (no cf) and with the crystal field splitting term (cf), and the corresponding experimental values for the cerium compounds. The experimental values of the crystal field splitting $\left(\Delta_{C F}\right)$ are also listed. All moments are given in units of $\mu_{B}$, the temperatures and the crystal field splitting are given in units of Kelvin. . . . . . . . . . . . .

3.11 Estimated uncertainty in the calculated zero-temperature ordered moments for the cerium compounds based on an uncertainty in the experimental values of the crystal field splitting used in this calculation in units of $\mu_{B} \ldots \ldots \ldots \ldots \ldots \ldots$ 
3.12 First nearest neighbor exchange interaction matrices for $\mathrm{CeAs}$ and $\mathrm{CeSb}$ in units of $\mathrm{K}$, shown separately for the hybridization (HYB) and pure Coulomb exchange (COUL) interactions. . . . . . . . . . . . 82

3.13 Ground state energies of the type-I and type-IA configurations of the cerium pnictides at $\mathrm{T}=0$. All units are Kelvin. . . . . . . . . . . .

4.1 Final self-consistent values of the $f$ state Racah parameters for the uranium compounds studied in units of mRy. . . . . . . . . . . .

4.2 Values of the calculated and experimental ${ }^{3,4,5}$ magnetic moments for the uranium chalcogenides and pnictides in units of $\mu_{B}$. Listed are the FPLMTO values for the spin moment $\mu_{S}$, the orbital moment $\mu_{L}$, and total moment $\mu$, for the spin polarized only calculation $(\mathrm{FP}+\mathrm{SP})$ and for the calculation with spin polarization and orbital polarization correc$\operatorname{tion}(\mathrm{FP}+\mathrm{SP}+\mathrm{OP}) \ldots \ldots \ldots \ldots \ldots$

4.3 Difference in energy of uranium systems converged with different directions of anisotropy. $E_{001}$ and $E_{111}$ are the total energies of the system spin and orbitally polarized along the $<001>$ and $<111>$ axes respectively. The units of the energy difference is given in $\mathrm{mRy}$. . . . . . . . . . . . 9

4.4 Listed are the self-consistent energies from the FPLMTO electronic structure calculations for the uranium compounds. Shown are the interstitial kinetic energy $K E_{\text {int }}$, the center of the occupied $f$ valence band $E_{\text {coofb }}$, the Fermi energy $E_{F}$ and the resonance $f$ state energy $E_{f r e s}$. All energies are given in Rydbergs. . . . . . . . . . . . . . . . 95

4.5 Results of the self-consistent supercell calculations of the parameters entering into the two-ion interaction. Listed are the energy difference $E_{f}-E_{F}$, and the on-site Coulomb repulsion energy $U$. All units given are in $\mathrm{eV} . \ldots \ldots \ldots \ldots \ldots \ldots \ldots \ldots$ 
4.6 Characteristic matrix elements of the $10 \mathrm{X} 10$ two-ion interaction matrix $\left(E_{m_{a} m_{a}^{\prime}}^{m_{b} m_{b}^{\prime}}\left(\mathbf{R}_{\mathbf{b}}-\mathbf{R}_{\mathbf{a}}\right)\right)$ of the $f^{3}$ multiplet. Values of the hybridization induced $\left(E_{V^{4}}\right)$, cross terms $\left(E_{V^{2} J}\right)$, and pure Coulomb exchange $\left(E_{J^{2}}\right)$ interactions are listed for the $m=m^{\prime}=\frac{5}{2}$ elements for each of the first, second, and third nearest uranium neighbors in units of K. . . . . . 95

4.7 Calculated (from the model hamiltonian method) and experimental values of the ordering temperatures and ordered moments of the uranium compounds. The ordering temperature listed is the Curie temperature for the chalcogenide compounds and the Néel temperature in the case of the pnictide compounds. Temperatures are listed in degrees Kelvin, ordered moments are given in $\mu_{B} . \ldots \ldots \ldots \ldots \ldots \ldots$

4.8 Total two-ion interaction matrices for the first and second nearest neighbors of US given in units of K. . . . . . . . . . . . . . . . . . . 101

4.9 Average Scaled Exchange Parameters $\mathcal{J}$ calculated for both the results from the model hamiltonian method (MODL) and experiment (EXPT). These are listed in units of $K / \mu_{B}^{2} \ldots \ldots \ldots \ldots$. . . . . . 102 


\section{Chapter 1}

\section{INTRODUCTION}

The understanding of the magnetic properties of rare-earth and actinide series compounds has become one of the most interesting problems in solid state physics. ${ }^{1}$ The ions of the rare-earth series all have similar chemical properties because the outermost electron shells are in the $5 s^{2} 5 p^{6}$ configuration. The actinide compounds all have a $6 s^{2} 6 p^{6}$ configuration in their outer shells. The interesting magnetic characteristics of the rare-earth and actinide compounds arise from these outermost electron shells, however. These unusual properties are due to the presence of unfilled $f$ electrons shells in these compounds.

The radial extent of the outermost electrons in an atomic system is dictated by orthogonality to previously filled wave functions with the same orbital quantum number. In the case of the rare-earth atoms the $6 s$ states must be orthogonal to the $1 s, 2 s, 3 s, 4 s$ and $5 s$ states. Therefore, the radial wave function of these $6 s$ states has five nodes in it and is extended from the nucleus of the atom. When placed in a crystal structure, these electron states form the bands inside of the crystal. Those electrons in lower $s$ and $p$ orbitals form the atomic core of the system and are affected essentially by the atomic potential of the nucleus. However, the unfilled $4 f$ electrons behave differently. The $4 f$ states are the first states of orbital quantum number $l=3$ to be filled. Therefore, these are already orthogonal to all previous states due to the angular portion of the wave function. Thus, the radial wave function of these states has no nodes and the $f$ electrons remain in close proximity to the nucleus. Even in a metallic environment these $4 f$ states 
maintain their integrity and their atomic properties. Because of the small radial extent of these $f$ states, they are referred to as "localized" states. A similar behavior, though to a lesser extent due to orthogonality with the $4 f$ states, is found in the actinide series $5 f$ states.

The close proximity of these $f$ states to the nucleus causes the electrons to be highly correlated. Consider the addition of a second electron into a localized $f$ state. The electrons will now repel each other. Because of the small spatial extent of the $f$ wave function, the energy of these repulsions is large. Electron correlations are referred to as strong when this on-site Coulomb repulsion $U$ between electrons is much larger than the energies associated with the hybridization of orbitals belonging to different atoms. ${ }^{2}$ This hybridization is characterized in a solid by the resonance width $\Gamma$ of the $f$ energy band under consideration. For large values of $U / \Gamma$ these states do not form energy bands in a compound like the other valence electron states do. However, these remain localized at each atom site, and should be treated differently.

This study examines the magnetic properties of two classes of compounds: the cerium monopnictides and monochalcogenides, for which the $4 f$ states of the Ce atomic site remain highly localized; and uranium monopnictides and monochalcogenides, where the $5 f$ states of the uranium atomic sites range from almost localized to almost itinerant behavior. All of these compounds have simple isostructural rock-salt structure. (See Figure 1.1) These face-centered-cubic structures, with only two atoms in the primative unit cell, allow for detailed study and computational methods which would be cumbersome in more complicated systems. However, these compounds also have highly unusual magnetic properties caused by the felectron correlations. Therefore, not only are highly detailed studies of these compounds reasonable, they are also essential to understanding highly correlated systems.

In Table 1.1, we present the first principle calculated values for $U / \Gamma$ ( $\Gamma$ values from Ref 21, 25, and 26) for the compounds studied. Notice the distinctions between the different types of atoms discussed. This study encompasses a wide range of localization, from the well localized large cerium pnictides, to the more band like small uranium chalcogenides. 
Figure 1.1: Rock-salt structure found in the uranium and cerium pnictides and chalcogenides studied. The dark atoms are cation ( $\mathrm{Ce}$ or $\mathrm{U}$ ) sites and the light atoms indicate anion (pnictogen or chalcogen) sites. Also indicated are two directions the $<001>$ along the cube edge and the $<111>$ body diagonal.

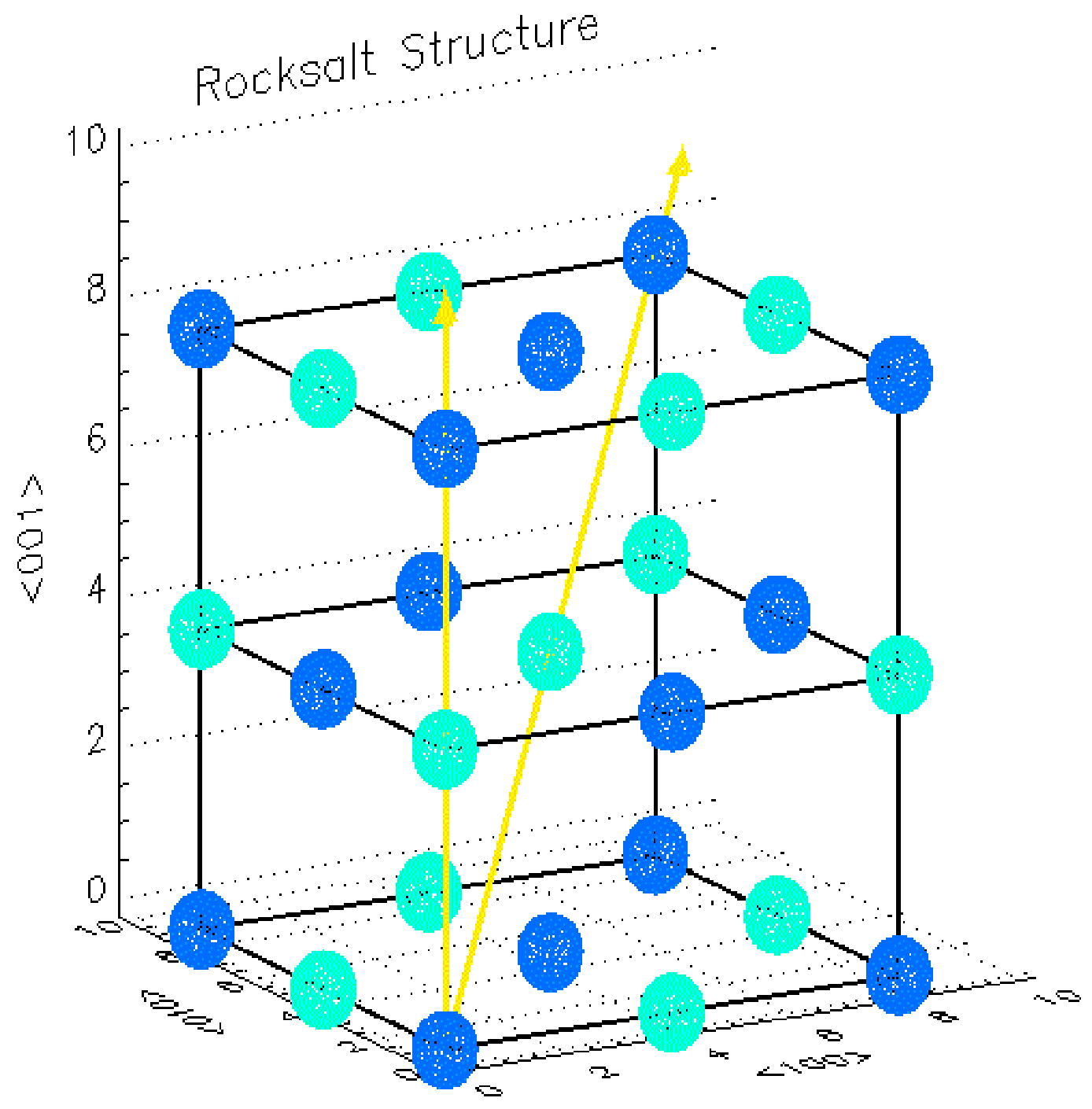


Throughout this study, we will be referring to certain distinctions in these compounds. Here we define them:

\section{CERIUM AND URANIUM COMPOUNDS}

This obvious difference in the two classes of compounds studied manifests itself in the $f$ electron states themselves. We must take two distinctions into account when dealing with the two classes of compounds. First, the $4 f$ states of the cerium compounds are highly localized, however, the uranium $5 f$ states are more extended in nature and therefore must be placed under different consideration than their cerium $f$ state counterparts. Second, the uranium compounds have three $f$ electrons in their ground state whereas the cerium compounds only have one. The calculations which involve a one electron picture, must be modified to take into account the $f^{3}$ multiplets of uranium.

\section{PNICTIDE AND CHALCOGENIDE SYSTEMS}

The pnictide systems are those compounds in which the rare earth or actinide atom containing the $f$ electron state is paired with an anion from column $\mathrm{V}-\mathrm{B}$ on a periodic table $(\mathrm{P}, \mathrm{As}, \mathrm{Sb}, \mathrm{Bi})$ and the chalcogenides are those in which the $f$ sites are paired with anions from column VI-B (S,Se,Te). The only chemical distinction between the two sets is the addition of a ligand $p$ electron in the chalcogenide systems. However, the ground state magnetic properties of the two sets of compounds are strikingly different. The ability for a first principles calculation of these magnetic distinctions between compounds which chemically only differ by one valence electron which, as we will observe, does not directly contribute to the magnetization, would be a huge success of that theory.

\section{LIGHT AND HEAVY COMPOUNDS}

Table 1.1: First principle calculated values of the ratio of Coulomb repulsion to resonance width, $U / \Gamma$, characteristic of the correlation between $f$ electrons in the uranium and cerium compounds studied. The quantities listed are dimensionless.

\begin{tabular}{|c|c|c|c|c|c|c|c|}
\hline & S & $\mathrm{Se}$ & $\mathrm{Te}$ & $P$ & As & $\mathrm{Sb}$ & $\mathrm{Bi}$ \\
\hline $\mathrm{U}$ & 7.2 & 9.9 & 13.8 & 18.3 & 20.9 & 28.4 & 28.5 \\
\hline $\mathrm{Ce}$ & 45 & 54 & 82 & 87 & 103 & 144 & 104 \\
\hline
\end{tabular}


The light (small anion size) compounds are those formed from the lighter anions such as sulfur or phosphorus. The heavy (large anion size) compounds are those formed from the heavier anions such as antimony or tellurium. The effect of the magnetic properties of this feature are not the weight, but the size of the anions. As the anion size increases, the separation between the atoms containing the $f$ states become more separated. The larger this separation becomes, the more localized an $f$ state will be. In our calculations, we attempt to predict the trend of the magnetic properties from the smaller, more itinerant systems, to the larger, more localized systems. 


\subsection{INFORMATION FROM EXPERIMENTS ON CERIUM COMPOUNDS}

The isostructural (rock-salt structure) series of the cerium monopnictides CeX (X $=\mathrm{P}$, $\mathrm{As}, \mathrm{Sb}, \mathrm{Bi})$ and monochalcogenides $(\mathrm{X}=\mathrm{S}, \mathrm{Se}, \mathrm{Te})$ have become prototype model systems for study, because of their unusual magnetic properties. This series of strongly correlated electron systems offer the opportunity to vary systematically, through chemical pressure, the lattice constant and the cerium-cerium separation on going down the pnictogen or chalcogen column, and hence tailor the degree of $4 f$ localization from the strongly localized limit in the heavier systems to to the weakly localized limit in the lighter systems. ${ }^{3-9}$ The sensitivity of the hybridization, Coulomb exchange, and crystalfield interactions to the chemical environment gives rise a variety of unusual magnetic properties.

This class of systems exhibits strong magnetic anisotropy which changes from the $<001>$ direction in the pnictides to the $<111>$ direction in the chalcogenides. The low temperature magnetic moment increases with increasing lattice constant for the pnictides from $0.80 \mu_{B}$ in $\mathrm{CeP}$ to $2.1 \mu_{B}$ in $\mathrm{CeBi}^{3-4}$ and decreases with increasing lattice constant for the chalcogenides from $0.57 \mu_{B}$ in $\mathrm{CeS}$ to $0.3 \mu_{B}$ in CeTe. ${ }^{5-7}$ The magnetic moment collapse from $\mathrm{CeSb}$ to $\mathrm{CeTe}$, with both systems having about the same lattice constant, is indicative of the sensitivity of the exchange interactions to the chemical environment. The ordering temperature increases from $8 \mathrm{~K}$ in $\mathrm{CeP}$ to $26 \mathrm{~K}$ in $\mathrm{CeBi}$ for the pnictides, whereas it decreases from $8.4 \mathrm{~K}$ in $\mathrm{CeS}$ to an unusually low $2.2 \mathrm{~K}$ in $\mathrm{CeTe}^{3-7}$

An unusual feature of the cerium series is the large suppression of the crystal field (CF) splitting of the $\mathrm{Ce}^{3+}$ free-ion $4 f_{5 / 2}$ multiplet from values expected from the behavior of the heavier isostructural rare-earth monopnictides. ${ }^{10}$ In both the cerium monopnictides and monochalcogenides, the $\mathrm{CF}$ splitting between the $\Gamma_{7}$ doublet and the $\Gamma_{8}$ quartet decreases with increasing anion size, from $150 \mathrm{~K}$ for $\mathrm{CeP}$ to $10 \mathrm{~K}$ in $\mathrm{CeBi}$ and from $130 \mathrm{~K}$ for $\mathrm{CeS}$ to $30 \mathrm{~K}$ for $\mathrm{CeTe}$, and it is about the same in both series, a rather surprising result in view of the additional valence electron on the chalcogen ion. ${ }^{11} \mathrm{Neu}-$ 
tron scattering experiments have shown ${ }^{12}$ that the $\Gamma_{7}$-doublet is the $\mathrm{CF}$ ground state in all the cerium monopnictides and monochalcogenides.

Given in Table 1.2 is a summary of the experimental results for the magnetic properties of the cerium compounds. 


\subsection{INFORMATION FROM EXPERIMENTS ON URANIUM COMPOUNDS}

Over the years the uranium monopnictides $\mathrm{UX}(\mathrm{X}=\mathrm{P}, \mathrm{As}, \mathrm{Sb}, \mathrm{Bi})$ and monochalcogenides $(\mathrm{X}=\mathrm{S}, \mathrm{Se}, \mathrm{Te})$ have been placed under careful scrutiny for their unusual magnetic properties and electronic structure. ${ }^{13}$ In this class of compounds, with the simple rock-salt structure, one can vary the uranium-uranium spacing ${ }^{14}$ and examine the change in magnetic behavior as the degree of $5 f$-electron localization changes from localized to itinerant behavior, as the chemical environment changes. This family of compounds also allows the study of the dependence of the magnetic behavior on the hybridization between the $5 f$ states of uranium and the uranium $6 d$ states or the ligand $p$ electrons when one replaces the pnictide with its corresponding chalcogenide, where the only apparent chemical change is the addition of a p-electron in the anion.

In these systems there is large directional magnetic anisotropy, which changes from anti-ferromagnetic ordering in the $\langle 001\rangle$ direction in the pnictides to ferromagnetic ordering in the $\langle 111\rangle$ direction in the chalcogenides. For the monopnictides, both the ordering temperature and ordered moment increase on going down the pnictide column from UP $\left(125 \mathrm{~K}\right.$ and $\left.1.8 \mu_{B}\right)$ to $\mathrm{UBi}\left(285 \mathrm{~K}\right.$ and $\left.3.0 \mu_{B}\right) \cdot{ }^{15} \mathrm{On}$ the other hand, for the chalcogenides, while the moment increases on going from US $\left(1.75 \mu_{B}\right)$ to UTe $\left(2.2 \mu_{B}\right)$, the Curie temperature decreases with increasing lattice constant (178K for US to $104 \mathrm{~K}$ for UTe). ${ }^{16,17}$

Table 1.2 gives the experimental values of the magnetic properties in this class of systems. 
Table 1.2: Summary of the experimental results for the various magnetic properties of the cerium compounds. Listed are the lattice constant $(a)$, the zero temperature ordered moment $\left(\mu_{0}\right)$, the ordering temperature $\left(T_{N}\right)$, the crystal field splitting $\left(\Delta_{C F}\right)$, and the direction of anisotropy.

\begin{tabular}{|c|c|c|c|c|c|}
\hline & $a(\AA)$ & $\mu_{0}\left(\mu_{B}\right)$ & $T_{N}(K)$ & $\Delta_{C F}(K)$ & $\hat{M}$ \\
\hline $\mathrm{CeS}$ & 5.78 & 0.57 & 8.4 & 140 & $<111>$ \\
$\mathrm{CeSe}$ & 5.99 & 0.57 & 5.4 & 120 & $<111>$ \\
$\mathrm{CeTe}$ & 6.35 & 0.30 & 2.2 & 32 & $<111>$ \\
\hline $\mathrm{CeP}$ & 5.95 & 0.81 & 8 & 150 & $<001>$ \\
$\mathrm{CeAs}$ & 6.08 & 0.85 & 8 & 137 & $<001>$ \\
$\mathrm{CeSb}$ & 6.42 & 2.06 & 17 & 37 & $<001>$ \\
$\mathrm{CeBi}$ & 6.50 & 2.10 & 26 & 8 & $<001>$ \\
\hline
\end{tabular}

Table 1.3: Experimental results for the magnetic properties of the uranium compounds studied. Listed are the lattice constant $(a)$, the zero-temperature ordered moment $\left(\mu_{0}\right)$, the ordering temperature $\left(T_{\text {ord }}\right)$, and the direction of anisotropy $\hat{M}$ with the magnetic ordering. The ordering temperature is the Curie temperature for the chalcogenides, and the Néel temperature for the pnictides.

\begin{tabular}{|c|c|c|c|c|}
\hline & $a(\stackrel{\circ}{ })$ & $\mu_{0}\left(\mu_{B}\right)$ & $T_{\text {ord }}(K)$ & $\hat{M}$ \\
$\mathrm{US}$ & 5.49 & 1.6 & 178 & $\mathrm{fm}<111>$ \\
$\mathrm{USe}$ & 5.73 & 2.0 & 160 & $\mathrm{fm}<111>$ \\
$\mathrm{UTe}$ & 6.15 & 2.2 & 104 & $\mathrm{fm}<111>$ \\
\hline $\mathrm{UP}$ & 5.59 & 1.8 & 125 & af $<001>$ \\
$\mathrm{UAs}$ & 5.78 & 2.2 & 126 & af $<001>$ \\
$\mathrm{USb}$ & 6.19 & 2.8 & 215 & af $<001>$ \\
$\mathrm{UBi}$ & 6.34 & 3.0 & 285 & af $<001>$ \\
\hline
\end{tabular}




\subsection{MOTIVATION AND APPROACH}

The purpose of this work is to understand the underlying mechanisms responsible for the unusual magnetism in these classes of systems and to investigate the interplay of the various exchange interactions. We have employed two $a b$ initio based approaches to calculate the magnetic properties of these compounds. First, we have carried out $a b$ initio spin polarized electronic structure calculations based on the full potential linear muffin tin (FPLMTO) method $^{18}$ using 1) only the spin polarization, with the orbital polarization included through spin-orbit coupling, and 2) both the spin and orbital polarization correction. ${ }^{19}$ Second, we apply an $a b$ initio based approach synthesizing 1) a phenomenological theory of orbitally driven magnetism based on the Anderson and Kondo lattice model which incorporates explicitly the hybridization induced and the Coulomb exchange interactions on an equal footing, and 2) FPLMTO electronic structure calculations allowing a first principles evaluation of all the parameters entering the model Hamiltonian. In the case of the cerium compounds, we also include the crystal field interactions on an equal footing with the hybridization and Coulomb exchange. To date, our attempts ${ }^{20}$ to determine the CF splitting for the cerium compounds studied on a fully $a b$ initio basis have not proven successful, and thus we use the experimental ${ }^{12}$ CF value for each compound. Unfortunately, because of technical difficulties and a lack of experimental information, we are unable to use this treatment of the crystal field for the uranium compounds.

For the cerium compounds, comparison of the two methods will test the limitations of these $a b$ initio based calculations as the degree of $4 f$ localization is varied across the series, and will reveal the failure of standard $a b$ initio electronic structure calculations based on density functional theory within the local density approximation to predict, even qualitatively, the trend of magnetic properties in these strongly correlated electron systems. In the past, we have applied the second method to investigate the effect of hybridization-induced exchange interactions ${ }^{21-22}$ and the effect of both the hybridization and Coulomb exchange interactions ${ }^{23}$ on the magnetic properties of the heavier cerium pnictides and chalcogenides (CeBi, CeSb, and $\mathrm{CeTe}$ ). However, these calculations did not 
take into account the crystal field interaction and employed a warped muffin-tin LMTO calculation for the parameters entering the model. The very good agreement found ${ }^{23}$ with experiment for the low-temperature magnetic moment and ordering temperature is due to the fact that the CF interaction in the heavier systems is smaller (about $8 \mathrm{~K}$ ) than the exchange interactions. We have generalized our ab initio based method to include the exchange (both hybridization and Coulomb exchange) and crystal field interactions on an equal footing and to employ a full potential LMTO evaluation of the exchange parameters. We find that the effect of the full potential on both the hybridization and Coulomb exchange interactions is small. Once the regime of magnetic behavior is determined by the primary effect of the hybridization and coulomb exchange interactions, the inclusion of the $\mathrm{CF}$ interaction will be shown to play an important role as one tunes the degree of $4 f$ localization across this series of strongly correlated electron systems.

In the case of the widely ranging $5 f$ localization of the uranium compounds, both methods provide a limited degree of success within the regime for which each is most appropriately suited. The pure electronic structure band calculations give good agreement with experiment for the lighter uranium compounds, whereas the model hamiltonian approach provides better agreement for the heavier uranium compounds. These results are consistent with the good agreement found in previous calculations. ${ }^{24}$ However, neither approach gives good agreement for the full spectrum of uranium compounds, demonstrating the limitations of both. 


\section{Chapter 1 References}

1. C. Kittel, Introduction to Solid State Physics 7th edition (John Wiley \& Sons, Inc. 1996).

2. P. Fulde, Electron Correlations in Molecules and Solids (Springer-Verlag 1991).

3. J. Rossat-Mignod, P. Burlet, S. Quezel, J. M. Effantin, D. Delacôte, H. Bartholin, O. Vogt, and D. Ravot, J. Magn. Magn. Mater. 31-34, 398 (1983).

4. B. R. Cooper, R. Siemann, D. Yang, P. Thayamballi, and A. Banerjea, in The Handbook of the Physics and Chemistry of the Actinides, edited by A. J. Freeman and G. H. Lander (North-Holland, Amsterdam, 1985), Vol. 2, Chap. 6, pp. 435500 .

5. A. Dönni, A. Furrer, P. Fischer, and F. Hulliger, Physica B 186-188, 541 (1993).

6. F. Hulliger, B. Natterer, and H. R. Ott, J. Magn. Magn. Mater. 8, 87 (1978).

7. H. R. Ott, J. K. Kjems, and F. Hulliger, Phys. Rev Lett. 42, 1378 (1979).

8. J. Rossat-Mignod, J. M. Effantin, P. Burlet, T. Chattopadhyay, L. P. Regnault, H. Bartholin, C. Vettier, O. Vogt, D. Ravot, and J. C. Achart, J. Magn. Magn. Mater. 52, 111 (1985).

9. N. Môri, Y. Okayama, H. Takahashi, Y. Haga, and T. Suzuki, Physica B 186-188, $444(1993)$.

10. R. J. Birgeneau, E. Bucher, J. P. Maita, L. Passell, and K. C. Turberfield, Phys. Rev. B 8, 5345 (1973).

11. H. R. Ott, J. K. Kjems, and F. Hulliger, Phys. Rev. Lett. 42, 1378 (1979); F. Hulliger and H.R. Ott, J. Phys. (Paris) Colloq. 40, C5, 128 (1979).

12. J. Rossat-Mignod, J. M. Effantin, P. Burlet, T. Chattopadhyay, L. P. Regnault, H. Bartholin, C. Vettier, O. Vogt, D. Ravot, and J.C. Achart, J. Magn. Magn. Mater. 52111 (1985). 
13. H. Rudiger, H. R. Ott, and O. Vogt, Physica B 130, 538 (1985).

14. B. Reihl, N. Martensson, and O. Vogt, J. Appl. Phys. 53, 2008 (1982).

15. S. K. Sinha, G. H. Lander, S. M. Shapiro, and O. Vogt, Phys. Rev. 23, 4556 (1981).

16. T. M. Holden, W. J. L. Buyers, E. C. Svensson, J. A. Jackman, A. F. Murray, O. Vogt, and P. de. V. DuPlessis, J. Appl. Phys. 53, 1967 (1982).

17. T. M. Holden, W. J. L. Buyers, P. de. V. DuPlessis, K. M. Hughes, and M. F. Collins, J. Magn. Magn. Mater. 54-57, 1175 (1986).

18. D. L. Price and B.R. Cooper, Phys. Rev. B 39, 4945 (1989).

19. O. Eriksson, M. S. S. Brooks, and B. Johansson, Phys. Rev. B 41, 7311 (1990).

20. N. Kioussis, private communication.

21. J. M. Wills and B. R. Cooper, Phys. Rev. B 36, 3809 (1987).

22. N. Kioussis, B. R. Cooper, and J. M. Wills, Phys. Rev. B 44, 10003 (1991).

23. Q. G. Sheng and B. R. Cooper, J. Appl. Phys. 69, 5472 (1991); Phys. Rev. B 50,965 (1994).

24. Q. G. Sheng, B. R. Cooper, and S. P. Lim, Phys. Rev. B 50, 9215 (1994).

25. N. Kioussis, D. Swearingen, B. R. Cooper, and J. M. Wills, J. Appl. Phys. 69, 5475 (1991).

26. N. Kioussis, H. J. Yu, B. R. Cooper, Q. G. Sheng, and J. M. Wills, J. Appl. Phys. 73,5424 (1993). 


\section{Chapter 2}

\section{METHODS OF SOLUTION}

We set out to understand what affects the magnetic properties of the cerium and uranium monopnictides and monochalcogenides by employing methods to calculate the magnetic properties of these compounds from first principles. We begin with the many-body Hamiltonian, describing an electron system interacting with an external potential.

$$
H=\sum_{i=1}^{N}\left(-\frac{1}{2} \nabla_{i}^{2}+V_{i}(\mathbf{r})\right)+\frac{1}{2} \sum_{i \neq j} \frac{1}{\left|\mathbf{r}_{\mathbf{i}}-\mathbf{r}_{\mathbf{j}}\right|}
$$

where $V_{i}$ is the external potential and the second sum represents the mutual Coulomb interaction $V_{i j}$. Here we adopt atomic units where $\hbar=e=m_{e}=1$. What is needed is a method by which one can solve this hamiltonian for the system in question, using reasonable approximations. From those solutions we must then be able to extract the pertinent magnetic properties. Comparison of these results with experiment gives us a test of the validity and importance of the approximations and assumptions made in order to solve this problem within a reasonable amount of time.

There are two general approaches explored in this paper. First, a spin polarized full potential linear muffin tin orbital (FPLMTO) electronic structure calculation based on density functional theory within the local density approximation (LDA) is employed. We

perform two types of these calculations: one using only spin polarization with orbital polarization arising through spin-orbit coupling, and the other including an explicit orbital polarization correction. Second, a synthesis of phenomenology based on the Anderson-Kondo hamiltonian which incorporates the hybridization exchange and band-f 
exchange on an equal footing, and utilizes the FPLMTO electronic structure calculations to obtain a first principles evaluation of the parameters entering the hamiltonian. For cerium compounds we also examine a crystal field effect treated on an equal footing with the exchange interactions. 


\subsection{THE FPLMTO METHOD}

\subsubsection{DERIVATION}

Two theorems by Hohenberg and Kohn provide the groundwork for the calculations within density functional theory ${ }^{1}$. The first one states that in an external potential $V(\mathbf{r})$, the total energy of $\mathrm{n}$ electrons can be written as a functional of the electron charge density $\rho(\mathbf{r})$ as

$$
E[\rho]=\int V(\mathbf{r}) \rho(\mathbf{r}) d^{3} r+F[\rho]
$$

where here $F[\rho]$ is an unknown functional of $\rho$ only and is independent of $V$. The second theorem states that the ground state density of the system is that which minimizes $E[\rho]$. Proofs of these theorems are given in Reference 1.

To apply this theory one makes approximations of the unknown $F[\rho]$ and divide it as follows

$$
F[\rho]=\frac{1}{2} \int d^{3} r d^{3} r^{\prime} \frac{\rho(\mathbf{r}) \rho\left(\mathbf{r}^{\prime}\right)}{\left|\mathbf{r}-\mathbf{r}^{\prime}\right|}+T_{0}[\rho]+E_{x c}[\rho] .
$$

The first term describes the Coulomb interaction. $T_{0}[\rho]$ is the kinetic energy of a system of noninteracting electrons. The last term is the exchange and correlation energy which contains the portion of the kinetic energy term not included in $T_{0}$ as well as the remaining exchange and correlation energies.

To minimize $E$ the charge density must satisfy the variational equation

$$
\int d^{3} r \delta \rho(\mathbf{r})\left\{V(\mathbf{r})+\int d^{3} r^{\prime} \frac{\rho\left(\mathbf{r}^{\prime}\right)}{\left|\mathbf{r}-\mathbf{r}^{\prime}\right|}+\frac{\delta T_{0}[\rho]}{\delta \rho(\mathbf{r})}+\frac{\delta E_{x c}[\rho]}{\delta \rho(\mathbf{r})}\right\}=0
$$

under the constraint $\int d^{3} r \delta \rho(\mathbf{r})=0$ required for total charge conservation. The above variational equation is the same as that of a noninteracting electron system with an effective potential given by

$$
V_{e f f}(\mathbf{r})=V(\mathbf{r})+\int d^{3} r^{\prime} \frac{\rho\left(\mathbf{r}^{\prime}\right)}{\left|\mathbf{r}-\mathbf{r}^{\prime}\right|}+v_{x c}(\mathbf{r}) .
$$

Here the exchange-correlation potential is defined as

$$
v_{x c}=\frac{\delta E_{x c}[\rho]}{\delta \rho(\mathbf{r})}
$$


Thus, to solve for the charge density we must solve the one-electron Schrödinger equation of the form

$$
\left(-\frac{1}{2} \nabla^{2}+V_{e f f}(\mathbf{r})\right) \psi_{\mu}(\mathbf{r})=\mathcal{E}_{\mu} \psi_{\mu}(\mathbf{r})
$$

This gives as a ground state energy

$$
E[\rho]_{\min }=\sum_{o c c} \mathcal{E}_{\mu}-\frac{1}{2} \int d^{3} r d^{3} r^{\prime} \frac{\rho(\mathbf{r}) \rho\left(\mathbf{r}^{\prime}\right)}{\left|\mathbf{r}-\mathbf{r}^{\prime}\right|}+E_{x c}[\rho]-\int d^{3} r v_{x c}(\mathbf{r}) \rho(\mathbf{r})
$$

where the first sum is over all occupied states.

The term in the above equations which is the most difficult to calculate is the exchange-correlation term. To deal with this term the local-density approximation is used. The exchange-correlation energy contribution from any given point in space is approximated by the exchange-correlation energy of a homogeneous electron gas with a density equal to that of the point in question, in other words the local density. Then the total exchange correlation is a sum of all the local contributions

$$
E_{x c}[\rho]=\int d^{3} r \rho(\mathbf{r}) \mathcal{E}_{x c}(\rho(\mathbf{r}))
$$

Here the $\mathcal{E}_{x c}(\rho(\mathbf{r}))$ is the exchange correlation energy per electron of a homogeneous gas of density $\rho$. The exchange potential becomes

$$
v_{x c}(\mathbf{r})=\frac{d\left(\rho(\mathbf{r}) \mathcal{E}_{x c}(\rho(\mathbf{r}))\right)}{d \rho(\mathbf{r})}
$$

and depends only on $\rho(\mathbf{r})$. With this approximation the $V_{\text {eff }}(\mathbf{r})$ also only depends on $\rho(\mathbf{r})$ and thus the Schrödinger equation becomes much simpler to solve. When performing spin-polarized calculations we use a form of the exchange correlation functional which depends on the spin-up and spin-down charge densities, $\rho_{\uparrow}$ and $\rho_{\downarrow}$. The parameterization of the functional by Vosko, Wilk and Nusair ${ }^{2}$ is used in our calculations.

Having a starting hamiltonian we now turn to forming a set of basis states in which to solve the hamiltonian. The linear-muffin-tin orbital method (LMTO) provides us with a useful set of basis functions because they are constructed from solutions to the atomic problem and are therefore close to the real solutions to the crystalline problem. This method divides space into non-overlapping muffin-tin spheres surrounding each 
atom site, and an interstitial region between the muffin tin spheres. For a full potential calculation (FPLMTO) we write the potential inside the spheres as a sum over lattice harmonics.

$$
V_{\alpha}(\mathbf{r})=\sum_{h} V_{\alpha, h}(\mathbf{r}) D_{\alpha, h}(\hat{\mathbf{r}})
$$

where the lattice harmonics, $D_{\alpha, h}(\hat{\mathbf{r}})$, are linear combinations of spherical harmonics that are invariant under point-group operations and $\alpha$ runs over the inequivalent spheres in a unit cell. In the interstitial region, the potential is expressed as a Fourier series,

$$
V_{i}(\mathbf{r})=\sum_{\mathbf{G}} e^{i \mathbf{G} \cdot \mathbf{r}} V(\mathbf{G})
$$

where the $\mathbf{G}$ are the reciprocal lattice vectors. The sum is truncated for $\mathrm{G}$ values larger than some cutoff magnitude, $G_{b \max }$. We set the average potential in the interstitial region to be zero.

We now construct the basis states which are Bloch sums,

$$
\Psi_{\kappa, \alpha, l, m, s}^{\mathrm{k}}(\mathbf{r})=\sum_{\mathbf{R}} e^{i \mathbf{k} \cdot \mathbf{R}} \Phi_{\kappa, \alpha, l, m, s}^{\mathrm{k}}(\mathbf{r}-\mathbf{R})
$$

The "muffin-tin orbitals" $\Phi$ are given by

$$
\begin{aligned}
\Phi_{\kappa, \alpha, l, m, s}^{\mathrm{k}}= & {\left[\mathcal{A} \phi_{\kappa, \alpha, l}\left(r_{\alpha}\right)+\mathcal{B} \dot{\phi}_{\kappa, \alpha, l}\left(r_{\alpha}\right)\right] Y_{l m}\left(\hat{\mathbf{r}_{\alpha}}\right) \chi_{s} \Theta\left(r_{\alpha} / s_{\alpha}\right) } \\
& +\left[-\kappa^{l+1} n_{l}(\kappa r)\right] Y_{l m}(\hat{\mathbf{r}}) \chi_{s} \Lambda(\mathbf{r}) \\
& +\sum_{\alpha^{\prime} l^{\prime} m^{\prime}}\left[\mathcal{C} \phi_{\kappa, \alpha^{\prime}, l^{\prime}}\left(r_{\alpha^{\prime}}\right)+\mathcal{D} \dot{\phi}_{\kappa, \alpha^{\prime}, l^{\prime}}\left(r_{\alpha^{\prime}}\right)\right] S_{\alpha^{\prime}, l^{\prime}, m^{\prime} ; \alpha, l, m}^{\mathrm{k}, \kappa} Y_{l^{\prime} m^{\prime}}\left(\hat{\mathbf{r}^{\prime}}\right) \chi_{s} \Theta\left(r_{\alpha^{\prime}} / s_{\alpha^{\prime}}(2.14)\right.
\end{aligned}
$$

In this equation $\mathbf{r}_{\alpha}=\mathbf{r}-\rho_{\alpha}$ is the position relative to the center of the $\alpha$ th muffintin. Here the step functions, $\Theta\left(r_{\alpha} / s_{\alpha}\right)$ and $\Lambda(\mathbf{r})$ are defined to be equal to 1 inside the $\alpha$ th muffin-tin and zero elsewhere; and 1 in the interstitial region and zero elsewhere respectively. Therefore, the first term is in the region of the $\alpha$ th muffin tin, the second term is the interstitial, and the last term is the contribution from all other spheres. The $s_{\alpha}$ 's are the muffin-tin radii of the $\alpha$ th muffin-tin. The functions $\phi(r)$ are solutions of the semirelativistic Dirac equation and the $\dot{\phi}(r)$ are the energy derivatives of these evaluated at chosen energy parameters $E_{\kappa, \alpha, l}$. The coefficients, $\mathcal{A}-\mathcal{D}$ are chosen so the basis states and their first derivatives are continuous at the muffin-tin boundaries. The 
$Y_{l m}$ are spherical harmonic and the $n_{l}(\kappa r)$ is a Neuman function with kinetic energy $\kappa^{2}$ (or a Hankel function if $\kappa^{2}$ is negative). The $S_{\alpha^{\prime}, l^{\prime}, m^{\prime} ; \alpha, l, m}^{\mathrm{k}, \kappa}$ are the KKR structure functions. ${ }^{3}$ The $\chi_{s}$ is the appropriate Dirac spinor. Each value of the parameter $\kappa$ is associated with an "energy window", allowing for the coverage of the various subbands. The energy parameters $E_{\kappa, \alpha, l}$ are set appropriately for each basis and each subband. The $\kappa$ and $E$ parameters are calculated after each iteration in the self-consistent procedure. The sum over $l^{\prime}$ in the third term of the basis state is in principle infinite; in practice we only keep terms up to $l=l_{\max }$, which varies depending upon the calculation. We use an all electron calculation in which the electrons are separated into bound, "core", electrons and free, "valence", electrons. For the valence electrons, the solutions are formed from the FPLMTO basis states above. The solutions of the "core" electron states are those of a full Dirac equation in the spherically averaged potential in each sphere.

The eigenstates take on the form

$$
\phi^{\mathrm{k}}(\mathbf{r})=\sum_{\kappa \alpha l m s} A_{\kappa, \alpha, l, m, s}^{\mathrm{k}} \Phi_{\kappa, \alpha, l, m, s}^{\mathrm{k}}(\mathbf{r})
$$

where the $A_{\kappa, \alpha, l, m, s}$ are the eigenvectors.

The method itself is implemented self-consistently. Beginning with a starting potential $V_{n}(\mathbf{r})$ the muffin-tin orbitals are constructed along with the Hamiltonian and overlap matrices. The program then solves for the eigenvectors, $A_{\kappa, \alpha, l, m, s}$, and thus forms the eigenfunctions $\psi^{\mathrm{k}}(\mathbf{r})$. From this the charge density is calculated via

$$
\rho(\mathbf{r})=\sum_{o c c}\left|\psi^{\mathrm{k}}(\mathbf{r})\right|^{2}+\text { "core" charges }+ \text { nuclei charges. }
$$

Then the new potential is calculated from Coulomb's law.

$$
\nabla^{2} \Phi=-4 \pi \rho(\mathbf{r})
$$

and adding the exchange-correlation potential for the new charge density.

$$
V^{\prime}=\Phi+v_{x c}(\rho)
$$

The new potential is then "mixed" with a combination of the previous potentials $V_{n+1}=f\left(V_{1}, V_{2}, \ldots, V_{n}, V^{\prime}\right)$ using the Broyden's jacobian updating method. ${ }^{5}$ This is then 
repeated using the new potential. Once the difference in the input and output potentials is small enough then the potential has "converged". We can be assured by the uniqueness theorem that the potential that solves both equations is the true potential to within a constant.

\subsubsection{ORBITAL POLARIZATION}

Using a parameterization introduced by Brooks, ${ }^{6}$ explicit orbital polarization is taken into account by means of an eigenvalue shift, $\Delta V_{m}=-E^{3} L_{z} m_{l}$, at each atom site. Here, $\mathrm{L}_{z}$ is the $\mathrm{z}$-component of the total orbital moment of the atom, $\mathrm{m}_{l}$ is the magnetic quantum number, and $\mathrm{E}^{3}$ is the Racah parameter which is evaluated at each iteration for a self-consistent calculation.

\subsubsection{SPECIFICS}

We now set about the task of calculating the magnetic properties for our systems. The systems are relatively simple, all only containing two independent muffin-tins, and an face-centered-cubic structure. We use one energy window for the direct calculation of the zero-temperature ordered moments for these compounds. The basis states represent the states occupied by the valence electrons and unfilled states near in energy to these valence states. For example, the potential for US is converged with $\mathrm{U} 7 s, 7 p, 6 d, 5 f$ and $\mathrm{S} 4 s, 3 p, 3 d$ basis states. For the cerium compounds the cerium basis states are the $6 s, 6 p, 5 d, 4 f$ states. The basis states for the uranium and cerium do not change across their respective series. The FPLMTO basis used for the ligands depend upon the type of the ligand. All other states are placed into the core and are not considered in the calculation of the magnetic properties. The $\kappa$ associated with the energy window is set to the squareroot of the interstitial kinetic energy. The energy parameters for all of the states $E_{\kappa, \alpha, l}$ are chosen to be at the muffin-tin-sphere-energy-window $l$-projected energies (except in cases where they were changed in order to enforce these states to be orthogonal to the "core" states).

The set of k-points used to converge the potentials is chosen by the special k-point method. ${ }^{7}$ This method allows for simple integration over the irreducible Brillouin zone for 
Table 2.1: Ligand basis states for the pure FPLMTO electronic structure calculations.

\begin{tabular}{|c|c|}
\hline LIGANDS & BASIS \\
\hline $\mathrm{P}$ and $\mathrm{S}$ & $4 s, 3 p, 3 d$ \\
$\mathrm{As}$ and $\mathrm{Se}$ & $5 s, 4 p, 4 d$ \\
$\mathrm{Sb}$ and $\mathrm{Te}$ & $6 s, 5 p, 5 d$ \\
$\mathrm{Bi}$ & $7 s, 6 p, 6 d$ \\
\hline
\end{tabular}

calculation of various properties which can be done during each iteration of the potential. For more detailed Brillouin Zone integration we use a tetrahedral k-point mesh. ${ }^{3}$ In this way we perform integrations over the Brillouin Zone and obtain the values for the spin, orbital and total polarization for these compounds at zero-temperature. By converging potentials for several different polarization directions and comparing the energies of the converged systems we also determine the direction of anisotropy for these compounds. 


\subsection{THE MODEL HAMILTONIAN METHOD}

\subsubsection{DERIVATION OF THE MODEL HAMILTONIAN}

In a previous publication ${ }^{8}$, Sheng and Cooper provide a very thorough first principles derivation of the model hamiltonian describing $f$-electrons interacting with non- $f$ band electrons in which the quantities can be extracted from a FPLMTO band structure calculation. Here, that derivation is reiterated.

First, electric charge in the crystal is classified into two types with regard to atomic origin: (1) the nuclei and filled-shell electrons, (2) the valence electrons which are separated further into $f$ (partially filled transition shell) electrons and the outermost $s, p$, and $\mathrm{d}$ electrons forming the non-f conduction bands in the solid. We are only interested in the type 2 electrons. The remaining charges form a rigid-background on which the $f$ and non-f valence electrons interact. We thus replace $\sum_{i} V_{i}$ in equation 2.1 with the periodic "background" potential $V_{0}\left(\mathbf{r}_{i}\right)$ of the filled-shell and nuclear charges and obtain the hamiltonian:

$$
H=\sum_{i} \frac{1}{2} \nabla_{i}^{2}+V_{0}\left(\mathbf{r}_{i}\right)+\frac{1}{2} \sum_{i \neq j} \frac{1}{r_{i j}}
$$

where $r_{i j}$ stands for $\left|\mathbf{r}_{\mathbf{i}}-\mathbf{r}_{\mathbf{j}}\right|$. To obtain the model hamiltonian, this hamiltonian is second-quantized:

$$
H=<\Phi(\mathbf{r})\left|\nabla^{2}+V_{0}(\mathbf{r})\right| \Phi(\mathbf{r})>+\frac{1}{2}<\Phi\left(\mathbf{r}_{1}\right) \Phi\left(\mathbf{r}_{2}\right)\left|\frac{1}{r_{12}}\right| \Phi\left(\mathbf{r}_{2}\right) \Phi\left(\mathbf{r}_{1}\right)>
$$

where $\mid \Phi(\mathbf{r})>$ is the field operator of electronic states. We now treat the band states and non-band $\mathrm{f}$ states as distinct, as the Anderson Hamiltonian does, by separating the field $\mid \Phi(\mathbf{r})>$ into a band field $\mid \Phi_{B}(\mathbf{r})>$ and a non-band f-field $\mid \Phi_{F}(\mathbf{r})>$ :

$$
\begin{gathered}
\Phi(\mathbf{r})=\Phi_{B}(\mathbf{r})+\Phi_{F}(\mathbf{r}) \\
\Phi_{B}(\mathbf{r})=\sum_{\mathbf{k}} \phi_{\mathbf{k}} b_{\mathbf{k}} \\
\Phi_{F}(\mathbf{r})=\sum_{\mathbf{R} m} \psi_{m}(\mathbf{r}-\mathbf{R}) c_{m}(\mathbf{R})
\end{gathered}
$$

where $b_{k}$ is the annihilation operator of the band state $\phi_{k}$, and $k$ signifies both momentum and band index; $c_{m}$ is the annihilation operator of the local f-state $\psi_{m}(r) ; R$ stands 
for the atomic site; and $m$ is the index of a local state. Since most of the physics happens in the sub-space of the partially filled spin-orbital multiplet, we restrict the local f-states in this sub-space of total angular momentum $j$, and $m$ becomes the magnetic quantum number of the f-states.

We now expand the right hand side of equation 2.20. Since the plan is to utilize LDA electronic structure calculations to calculate these quantities, we must be careful about what is already contained within the LDA and which quantities must be further evaluated. The first term on the right hand side is of one-body type and is simple in nature. There are three terms:

$$
\text { (i) }<\Phi_{B}(r)\left|\nabla^{2}+V_{0}(r)\right| \Phi_{B}(r)>=\sum_{k} \epsilon_{k}^{0} b_{k}^{+} b_{k}
$$

This is the bare band state energy and

(ii) $\left\langle\Phi_{F}(r)\left|\nabla^{2}+V_{0}(r)\right| \Phi_{F}(r)>=\sum_{R m} \epsilon_{m}^{0} c_{m}^{+}(R) c_{m}(R)\right.$

is the bare f-state energy. In writing this we have neglected the direct overlap between f-states centered on different sites, ie. the $R \neq R^{\prime}$ terms. We further approximate that all f-states with different quantum numbers have the same energy $E_{f}$ and neglect the effects of the crystal-field splitting and magnetic polarization for the f-states at this point.

$$
\text { (iii) }<\Phi_{B}(r)\left|\nabla^{2}+V_{0}(r)\right| \Phi_{F}(r)>+ \text { h.c. }=\sum_{k m R} V_{k m}^{0} e^{-i \mathbf{k} \cdot \mathbf{R}} b_{k}^{+} c_{m}(R)+\text { h.c. }
$$

This is the bare hybridization between band states and f-states. At this point we note that the above three quantities contain no corrections to the energies due to interactions from among the electrons, thus, we refer to these as being bare interactions.

We now evaluate the second term on the right hand side of equation 2.20 which is two body type. To make it easier to understand we write it in the form of an integral with the summation over spins being implicit in the integral operation:

$$
\begin{gathered}
\frac{1}{2}<\Phi\left(r_{1}\right) \Phi\left(r_{2}\right)\left|\frac{1}{r_{12}}\right| \Phi\left(r_{2}\right) \Phi\left(r_{1}\right)>= \\
\frac{1}{2} \iint\left[\Phi_{B}^{+}\left(r_{1}\right)+\Phi_{F}^{+}\left(r_{1}\right)\right]\left[\Phi_{B}\left(r_{1}\right)+\Phi_{F}\left(r_{1}\right)\right] \frac{1}{r_{12}}\left[\Phi_{B}^{+}\left(r_{2}\right)+\Phi_{F}^{+}\left(r_{2}\right)\right]\left[\Phi_{B}\left(r_{2}\right)+\Phi_{F}\left(r_{2}\right)\right] d r_{1}^{3} d r_{2}^{3}
\end{gathered}
$$

When this is expanded, we get terms (iv) through (xi):

$$
\text { (iv) } \frac{1}{2} \iint \Phi_{B}^{+}\left(r_{1}\right) \Phi_{B}\left(r_{1}\right) \frac{1}{r_{12}} \Phi_{B}^{+}\left(r_{2}\right) \Phi_{B}\left(r_{2}\right) d r_{1}^{3} d r_{2}^{3}
$$

This is the Coulomb interaction between non-f band electrons. This contains the direct 
Coulomb energy and the exchange correlation energy, both of which are included in the LDA calculation of the band state energy. Thus we combine this term with (i) and replace the bare band energy in the hamiltonian with the LDA band energy $\epsilon_{k}$.

$$
\text { (v) } \frac{1}{2} \iint \Phi_{F}^{+}\left(r_{1}\right) \Phi_{F}\left(r_{1}\right) \frac{1}{r_{12}} \Phi_{F}^{+}\left(r_{2}\right) \Phi_{F}\left(r_{2}\right) d r_{1}^{3} d r_{2}^{3}
$$

This term represents the Coulomb interaction between local f-electrons. By neglecting the inter-atomic part $\left(R \neq R^{\prime}\right)$ which is very small and the exchange Coulomb effects, this term becomes the on-site Coulomb repulsion of f-state electrons.

$$
\frac{U}{2} \sum_{R, m \neq m^{\prime}} n_{m}(R) n_{m^{\prime}}(R)
$$

$$
\begin{aligned}
& \text { (vi) } \iint \Phi_{B}^{+}\left(r_{1}\right) \Phi_{B}\left(r_{1}\right) \frac{1}{r_{12}} \Phi_{F}^{+}\left(r_{2}\right) \Phi_{F}\left(r_{2}\right) d r_{1}^{3} d r_{2}^{3} \\
& \text { (vii) } \iint \Phi_{B}^{+}\left(r_{1}\right) \Phi_{F}\left(r_{1}\right) \frac{1}{r_{12}} \Phi_{F}^{+}\left(r_{2}\right) \Phi_{B}\left(r_{2}\right) d r_{1}^{3} d r_{2}^{3}
\end{aligned}
$$

These are Coulomb interactions between band and non band f-electrons. (vi) is the direct Coulomb interaction and (vii) is the exchange Coulomb interaction. Written out these terms are,

$$
\sum_{k k^{\prime}} \sum_{m m^{\prime} R}\left[U_{m m^{\prime}}\left(\mathbf{k}, \mathbf{k}^{\prime}\right)-J_{m m^{\prime}}\left(\mathbf{k}, \mathbf{k}^{\prime}\right)\right] e^{i\left(\mathbf{k}-\mathbf{k}^{\prime}\right) \cdot \mathbf{R}} b_{k}^{+} b_{k^{\prime}} c_{m}^{+}(R) c_{m^{\prime}}(R)
$$

where $U_{m m^{\prime}}\left(\mathbf{k}, \mathbf{k}^{\prime}\right)$ is the direct Coulomb term

$$
U_{m m^{\prime}}\left(\mathbf{k}, \mathbf{k}^{\prime}\right)=\iint \phi_{k}^{*}\left(r_{1}\right) \phi_{k^{\prime}}\left(r_{1}\right) \frac{1}{r_{12}} \psi_{m}^{*}\left(r_{2}\right) \psi_{m^{\prime}}\left(r_{2}\right) d r_{1}^{3} d r_{2}^{3}
$$

and $J_{m m^{\prime}}\left(\mathbf{k}, \mathbf{k}^{\prime}\right)$ is the exchange Coulomb term

$$
J_{m m^{\prime}}\left(\mathbf{k}, \mathbf{k}^{\prime}\right)=\iint \phi_{k}^{*}\left(r_{1}\right) \psi_{m}^{*}\left(r_{2}\right) \frac{1}{r_{12}} \psi_{m^{\prime}}\left(r_{1}\right) \phi_{k^{\prime}}\left(r_{2}\right) d r_{1}^{3} d r_{2}^{3}
$$

In these integrals we have neglected the terms in which $R \neq R^{\prime}$, since $\psi_{m}(r)$ is very localized and also because the $\frac{1}{r_{12}}$ decays with distance so that the contribution to the terms is very small when $\psi_{m}\left(r_{1}\right)$ and $\psi_{m}\left(r_{2}\right)$ are centered on different lattice sites. Note that the lowest order effects of these Coulomb interactions are contained within the LDA approximation calculation of both the band energies, $\epsilon_{k}$, and the $f$-state energies, $E_{f}$. However, in the two-ion interaction, which is a higher ordered effect, the energy of an $f$ electron depends upon the state of another $f$ electron at another lattice site. This is not taken into account in the local density approximation, which only depends on the states 
at the lattice site containing the $f$ electron in question. So, we cannot neglect these terms when calculating the two-ion interaction. However, in the random phase approximation, the contribution to the two-ion interaction from the direct Coulomb term, $U_{m m^{\prime}}\left(\mathbf{k}, \mathbf{k}^{\prime}\right)$, is essentially the screened Coulomb interaction between two felectrons at different sites, which is very small even without the screening effects. Therefore, we neglect the direct Coulomb interaction from the calculation of the two-ion interaction and realize that the contributions to the LDA calculated energies are already included. The two ion interaction due to the exchange Coulomb interaction $J_{m m^{\prime}}\left(\mathbf{k}, \mathbf{k}^{\prime}\right)$ cannot be viewed in such a way and is not negligibly small. Thus we retain this term in the hamiltonian and also take into account the screening which is discussed in section 2.2.2.

$$
\begin{aligned}
& \text { (viii) } \iint \Phi_{B}^{+}\left(r_{1}\right) \Phi_{F}\left(r_{1}\right) \frac{1}{r_{12}} \Phi_{B}^{+}\left(r_{2}\right) \Phi_{B}\left(r_{2}\right) d r_{1}^{3} d r_{2}^{3}+\text { H.C. } \\
& \text { (ix) } \iint \Phi_{B}^{+}\left(r_{1}\right) \Phi_{F}\left(r_{1}\right) \frac{1}{r_{12}} \Phi_{F}^{+}\left(r_{2}\right) \Phi_{F}\left(r_{2}\right) d r_{1}^{3} d r_{2}^{3}+\text { H.C. }
\end{aligned}
$$

The net effect of these two terms are hybridization between band and local $f$ electrons. We approximate $\Phi_{B}^{+}\left(r_{2}\right) \Phi_{B}\left(r_{2}\right)$ as $<\rho_{B}\left(r_{2}\right)>$ and $\Phi_{F}^{+}\left(r_{2}\right) \Phi_{F}\left(r_{2}\right)$ as $<\rho_{F}\left(r_{2}\right)>$. Then the sum of (viii) and (ix) becomes

$$
\iint \Phi_{B}^{+}\left(r_{1}\right) \frac{\left\langle\rho_{B}\left(r_{2}\right)+\rho_{F}\left(r_{2}\right)\right\rangle}{r_{12}} \Phi_{F}\left(r_{1}\right) d r_{1}^{3} d r_{2}^{3}+\text { H.C. }
$$

Since $\frac{\left\langle\rho_{B}\left(r_{2}\right)+\rho_{F}\left(r_{2}\right)\right\rangle}{r_{12}}$ is included in the effective potential of the LDA we treat these terms by combining them with the bare hybridization and replace it with the LDA hybridization $V_{k m}$ in the model hamiltonian.

(x) $\frac{1}{2} \iint \Phi_{B}^{+}\left(r_{1}\right) \Phi_{F}\left(r_{1}\right) \frac{1}{r_{12}} \Phi_{B}^{+}\left(r_{2}\right) \Phi_{F}\left(r_{2}\right) d r_{1}^{3} d r_{2}^{3}$

$$
\text { (xi) } \frac{1}{2} \iint \Phi_{F}^{+}\left(r_{1}\right) \Phi_{B}\left(r_{1}\right) \frac{1}{r_{12}} \Phi_{F}^{+}\left(r_{2}\right) \Phi_{B}\left(r_{2}\right) d r_{1}^{3} d r_{2}^{3}
$$

The final two terms create or annihilate two local $f$ electrons. We neglect them because such processes occur at high energy.

From these terms we obtain the following model Hamiltonian:

$$
H=H_{0}+H_{1}
$$

where

$$
H_{0}=\sum_{k} \epsilon_{k} b_{k}^{+} b_{k}+\sum_{R m} E_{f} c_{m}^{+}(R) c_{m}(R)+\frac{U}{2} \sum_{R, m \neq m^{\prime}} n_{m}(R) n_{m^{\prime}}(R)
$$

and 


$$
H_{1}=\sum_{k m R}\left[V_{k m} e^{-i \mathbf{k} \cdot \mathbf{R}} b_{k}^{+} c_{m}(R)+H . C .\right]-\sum_{k k^{\prime}} \sum_{m m^{\prime} R} J_{m m^{\prime}}\left(\mathbf{k}, \mathbf{k}^{\prime}\right) e^{-i\left(\mathbf{k}-\mathbf{k}^{\prime}\right) \cdot \mathbf{R}} b_{k}^{+} c_{m}^{+}(R) b_{k^{\prime}} c_{m^{\prime}}(R)
$$

Here the $\epsilon_{k}$ are the conduction band energies, $E_{f}$ is the energy of a localized $f$-state, $U$ is the on-site Coulomb repulsion of localized $f$ electrons, $V_{k m}$ is the hybridization between localized $f$ and non- $f$ states, and $J_{m m^{\prime}}\left(\mathbf{k}, \mathbf{k}^{\prime}\right)$ is the band- $f$ exchange.

\subsubsection{AB INITIO CALCULATION OF PARAMETERS}

Having obtained the form of the model Hamiltonian we now set about the task of calculating the parameters entering into the hamiltonian from first principles relating them to the FPLMTO electronic structure calculations. Using the converged potential, we can calculate the parameters using a tetrahedral mesh of $89 \mathrm{k}$-points to perform the integrations over the Brillouin zone. ${ }^{3}$

To begin we converge potentials for the cerium and uranium systems from which we extract the parameters entering into the hamiltonian. We use a basis set consisting of the non- $f$ valence states as mentioned before. However, the treatment of the $f$ states varies depending upon the nature of the compound. For cerium compounds, these $f$ states are placed into the "core" and are treated as "atomic like". However, this treatment is inappropriate for the uranium compounds in which the $f$ states are more extended. For the uranium compounds the $5 f$ states are treated as band states and placed into the valence. In this way we take into account the self-banding of the $5 f$ states in uranium which is otherwise neglected by the model hamiltonian. Also, included in the valence are the highest filled $p$ states of uranium and cerium along with the highest filled $s$ states of the anion; these states are also referred to as "semi-core" states. This provides us with a more accurate calculation of the potential. Below are the details used in the calculation of each of the parameters entering into the model hamiltonian.

\section{A. THE BAND ENERGIES}

The band energies, $\epsilon_{k}^{(n)}$, are calculated directly by the FPLMTO self consistent electronic structure calculations. And no further calculation is required.

\section{B. F-STATE AND ON-SITE COULOMB REPULSION}


The $f$-state energy $E_{f}$ and the on-site Coulomb repulsion of local $f$ electrons, $U$ involve the effects of interconfigurational fluctuations of the $f$ states, such as $f^{n} \leftrightarrow f^{n \pm 1}$ which are important for $f$ electron phenomena. Therefore, these do not directly come from the LDA calculation.

To calculate these quantities we use three supercell calculations of the electronic structure of the system in question. ${ }^{9-11}$ The supercell consists of four cerium (or uranium) sites and four anion site forming a cube of length $\frac{a}{2}$, where $a$ is the lattice constant. This cell consists of a central cation site, placed at the origin, and three surrounding sites. The configuration of the central site is varied by adding or removing an $f$ electron from the core, while keeping the number of $f$ electrons in the core of the surrounding sites constant, ( $f^{1}$ configurations for cerium compounds and $f^{3}$ configurations for uranium compounds). We then obtain the "core" $f$-state energy $E_{f}^{n}$ and the Fermi energy $E_{F}^{n}$ for each configuration of the central atom site, (placing 0,1 , or $2 f$ electrons on cerium central atoms sites; and placing 2,3 , and $4 f$ electrons on uranium atom sites). For each configuration we then take the difference: $\mathcal{E}^{n}=E_{f}^{n}-E_{F}^{n}$. From linear transition theory we find that the energy required to place a localized $f$ electron in the band at the Fermi energy is $E_{F}-E_{f}=-\frac{1}{2}\left(\mathcal{E}^{n}+\mathcal{E}^{n-1}\right)$. The energy required to change from a $f^{n}$ to a $f^{n+1}$ configuration is $E_{F}+U-E_{f}=\frac{1}{2}\left(\mathcal{E}^{n}+\mathcal{E}^{n+1}\right)$. In these equations $n=1$ for cerium compounds and $n=3$ for uranium compounds. From these quantities, and from a self-consistent calculation of the Fermi energy $\left(E_{F}\right)$, we obtain both the local $f$-state energy, $E_{f}$ and the energy cost $(\mathrm{U})$ associated with adding one additional electron to the $f^{n}$ configuration due to the Coulomb repulsion of the $f$ electrons.

\section{HYBRIDIZATION}

The hybridization between a non $f$ band state $\Phi_{B}^{n}$ and a localized $f$ state $\Phi_{F}$ is,

$$
V_{m k}^{n}=<\Phi_{B}^{n}(r)|H| \Phi_{F}^{m}(r)>
$$

where $n$ is the band index. The calculation of $V_{m k}^{n}$ differs for cerium and uranium compounds because of the degree of the $f$ state localizations.

\section{Hybridization for Cerium Compounds}

For cerium compounds, as noted earlier, the $f$ electrons are placed into the "core" region 
and treated as "atomic-like". ${ }^{12}$ This treatment creates a well defined distinction between these localized $f$ states and all other non- $f$ valence states as the two groups are not allowed to hybridize during the convergence of the potential. In the FPLMTO calculation of this quantity, we require a good representation of the bands around the Fermi energy, $E_{F}$. Hence after the self-consistency process, the bands entering into this calculation are computed with the energy of the tails of the bases $\left(\kappa^{2}\right)$ set to the Fermi energy.

We represent the $f_{\frac{5}{2}}$ component of the $n$th band at wave vector $\mathbf{k}$ expanded in spherical waves about a $C e$ site at $\mathbf{R}=0$ in the unaugmented form

$$
\begin{aligned}
& C e<3, \frac{5}{2}, m_{j}\left|\psi^{n, \mathrm{k}}(r)>={ }_{C e}<3, \frac{5}{2}, m_{j}\right| \sum_{\kappa, \alpha, l, m, s} A_{\kappa, \alpha, l, m, s}^{n \mathrm{k}} \Phi_{\kappa, \alpha, l, m, s}^{n \mathrm{k}}(r)> \\
= & \sum_{\kappa, \alpha, l, m, s} \sum_{\alpha^{\prime}, l^{\prime}, m^{\prime}} C e<3, \frac{5}{2}, m_{j} \mid \Phi_{\alpha^{\prime}, l^{\prime}}^{J}\left(r_{\alpha^{\prime}}\right) Y_{l^{\prime} m^{\prime}}\left(\hat{\mathbf{r}^{\prime}}\right) \chi_{s} \Theta\left(\frac{r_{\alpha^{\prime}}}{s_{\alpha^{\prime}}}\right)>A_{\kappa, \alpha, l, m, s}^{n \mathrm{k}} S_{\alpha^{\prime}, l^{\prime}, m^{\prime} ; \alpha, l, m}^{\mathrm{k}, \kappa} \\
= & j_{3}\left(\kappa_{e f} r\right) \sum_{\kappa_{e f}, \alpha, l, m, s} \sum_{C e, 3, m^{\prime}}<3, \frac{5}{2}, m_{j} \mid l^{\prime}, m^{\prime}>\chi_{s} A_{\kappa, \alpha, l, m, s}^{n \mathrm{k}} S_{\alpha^{\prime}, l^{\prime}, m^{\prime} ; \alpha, l, m}^{\mathrm{k}, \kappa} .
\end{aligned}
$$

In the second step we note the fact that since there are no $f$ states in the basis, the portion of the LMTO wave functions inside of the muffin-tin at $R=0$ and the portion in the interstitial regions do not contribute to this expansion. We have written the radial wave function $\Phi_{\alpha^{\prime}, l^{\prime}}^{J}\left(r_{\alpha^{\prime}}\right)$ and note that in its unaugmented form is simply a spherical Bessel function as opposed to a linear combination of the radial wave equation $\phi$ and its energy derivative $\dot{\phi}$ which insures orthogonality to the core. To be consistent we should also calculate the non- $f$ band structure using this form. However, since the non- $f$ bands are well converged in the Cerium muffin-tin for $l \leq 2$ and without band- $f$ hybridization, the $l=3$ component has a negligible effect on these bands and we therefore ignore this. In other words, because of the nature of these states, they are deemed to already be orthogonal to the $f$ core states. Therefore, for simplicity, we use the above form to represent the hybridization with the $f$ state even though we calculate the non- $f$ bands using the augmented form of $\Phi_{\alpha^{\prime}, l^{\prime}}^{J}\left(r_{\alpha^{\prime}}\right)$.

The final form of this expansion is:

$$
<3, \frac{5}{2}, m_{j} \mid \psi^{n, \mathrm{k}}(r)>_{C e}=j_{3}(\kappa r) \mathcal{T}_{m_{j}}^{(n)}(\mathbf{k})
$$


Table 2.2: Previously calculated ${ }^{11,12} \mathrm{Ce} 4 f_{5 / 2}$ resonance widths, $\Gamma$, and hybridization potentials, $\nu\left(\kappa_{F}\right)$, in mRy calculated with equations (2.32) and (2.31) respectively and used in the calculation of the hybridization from the "core" point of view.

\begin{tabular}{|c|c|c|c|c|c|c|c|c|}
\hline & $\mathrm{CeS}$ & $\mathrm{CeSe}$ & $\mathrm{CeTe}$ & & $\mathrm{CeP}$ & $\mathrm{CeAs}$ & $\mathrm{CeSb}$ & $\mathrm{CeBi}$ \\
$\Gamma(\mathrm{mRy})$ & 9.72 & 8.33 & 5.67 & 4.99 & 4.28 & 3.06 & 4.23 \\
$\nu\left(\kappa_{F}\right)(\mathrm{mRy})$ & -4.40 & -3.92 & -3.08 & -3.25 & -2.97 & -2.41 & -2.77 \\
\hline
\end{tabular}

where, $\quad \mathcal{T}_{m_{j}}^{(n)}(\mathbf{k}) \equiv \sum_{m^{\prime}, s} \sum_{\alpha, l, m}<3, \frac{5}{2}, m_{j} \mid 3, m^{\prime}, s>A_{\kappa_{e f}, \alpha, l, m, s}^{n \mathrm{k}} S_{C e, 3, m^{\prime} ; \alpha, l, m}^{\mathrm{k}, \kappa_{e f}}$

The parameter entering into the model Hamiltonian $V_{k m}$ is a matrix element of the Hamiltonian for a single-particle potential surrounding a cerium site between the band states and the cerium $4 f_{\frac{5}{2}}$ state. Using the expansion above for the band states this matrix element takes on the form: ${ }^{12}$

$$
\begin{aligned}
V_{m k}^{n} & =\left\langle j_{3}|\mathcal{H}| \psi_{f}>\left[\mathcal{T}_{m}^{(n)}(\mathbf{k})\right]^{*}\right. \\
& =\nu\left(\kappa_{F}\right) \sqrt{\Omega}\left[\mathcal{T}_{m}^{(n)}(\mathbf{k})\right]^{*}
\end{aligned}
$$

where $\Omega$ is the unit cell volume.

Here, we have defined a hybridization potential

$$
\nu\left(\kappa_{F}\right)=-\left[\frac{\Gamma}{2 \kappa_{F} N \Omega}\right]^{1 / 2} .
$$

In this equation $N$ is a normalization factor obtained from performing the integration and for all cases $N \leq 1.02$. The resonance width $\Gamma$ is given by

$$
\Gamma=\frac{2}{\kappa_{F}} \frac{\left[\phi_{f}(s)\right]^{2}}{\left|h_{3}^{+}\left(\kappa_{F} s\right)\right|^{2}}
$$

where $\phi_{f}(s)$ is the resonant radial $f$ wave function evaluated at the cerium muffin-tin radius $\mathrm{s}$, and $h_{3}^{+}$is the $l=3$ spherical Hankel function of the first kind. The resonance widths and hybridization potentials, which were done in previous calculations ${ }^{12,13}$ are given in Table 2.2 for the cerium compounds.

\section{Hybridization for Uranium Compounds}

Since the $5 f$ states in uranium compounds are more delocalized than the $4 f$ states in 
cerium, we adopt a "band point of view" scheme to evaluate the hybridization matrix elements $V_{k m} \cdot{ }^{14}$ Implementing this procedure requires a Fourier transform of the core- $f$ bases states into band- $f$ bases. The two are related by

$$
\begin{gathered}
\psi_{k m}(r)=\frac{1}{\sqrt{N}} \sum_{R} e^{i k \cdot R} \psi_{m}(r-R) \\
\psi_{m}(r-R)=\frac{1}{\sqrt{N}} \sum_{R} e^{-i k \cdot R} \psi_{k m}(r)
\end{gathered}
$$

We separate the basis states in the FPLMTO scheme into two different types: $f$-band basis states $\psi_{k m}$ and the non- $f$-band basis $\phi_{k n}$. They are constructed in the same manner except for special choices of LMTO parameters are used for the $f$-band states. These states are distinguished by the orbital quantum number $l=3$, the energy parameter $E=E_{r}$ set to the resonance energy of the atomic $f$ state, and $\kappa^{2}$ the matching of the extended tail of the $f$ states to the non- $f$ valence band is chosen to be the center of the occupied valence band. We then orthogonalize the two sets by projecting out the $f$ components from the non- $f$ basis states.

We then calculate the elements of the Hamiltonian matrix according to the standard FPLMTO method. The fully hybridized Hamiltonian for a wave vector $k$ is

$$
H^{k}=\left[\begin{array}{cc}
H_{f}^{k} & V^{k} \\
V^{k \dagger} & H_{c}^{k}
\end{array}\right],
$$

where $H_{f}^{k}$ is the subblock of elements among $f$ bases, $H_{c}^{k}$ is the subblock of elements among the non- $f$ bases states, and $V^{k}$ is the subblock of elements between the $f$ and non- $f$ bases. These are defined as:

$$
\begin{gathered}
\left(H_{f}^{k}\right)_{m m^{\prime}}=<\psi_{k m}(r)|H| \psi_{k m^{\prime}}(r)> \\
\left(H_{c}^{k}\right)_{n n^{\prime}}=<\phi_{k n}(r)|H| \phi_{k n^{\prime}}(r)> \\
\left(V^{k}\right)_{m n}=<\psi_{k m}(r)|H| \phi_{k n}(r)>.
\end{gathered}
$$


What we need is to calculate the hybridization between the unhybridized $f$ and non- $f$ states. To do this we first suppress the hybridization in the full hamiltonian by setting $V^{k}=0$.

$$
H_{u n h}^{k}=\left[\begin{array}{cc}
H_{f}^{k} & 0 \\
0 & H_{c}^{k}
\end{array}\right]
$$

We then diagonalize this unhybridized hamiltonian to obtain the eigenvalues and eigenstates of the unhybridized $f$ and non- $f$ bands. The $f$-block $H_{f}^{k}$ has eigenvalues $\epsilon_{k}^{m}$ and eigenstates:

$$
\mid a_{k}^{m}>=\sum_{m^{\prime}} \alpha_{m m^{\prime}}^{k} \psi_{k m^{\prime}}(r)
$$

and the conduction block $H_{c}^{k}$ has eigenvalues $\epsilon_{k}^{n}$ and eigenstates:

$$
\mid b_{k}^{m}>=\sum_{n^{\prime}} \beta_{n n^{\prime}}^{k} \phi_{k n^{\prime}}(r)
$$

For the elements used in calculating the two-ion interaction we return to the core point of view. The $V_{k m}$ appearing in the model hamiltonian is the overlap between non- $f$-band eigenstates and local $f$ basis states:

$$
V_{k m}^{n}=<\psi_{m}(r)|H| b_{k}^{n}>
$$

Combining the above equations gives the result at $R=0$ :

$$
V_{k m}^{n}=\frac{1}{\sqrt{N}} \sum_{n^{\prime}} \beta_{n n^{\prime}}^{k}\left(V^{k}\right)_{m n^{\prime}}
$$

Here the $\left(V^{k}\right)_{m n^{\prime}}$ are from the fully hybridized hamiltonian.

\section{BAND-F COULOMB EXCHANGE}

The scheme we use to calculate the band-f exchange Coulomb interaction, $J_{n, n^{\prime}}\left(\mathbf{k}, \mathbf{k}^{\prime}\right)$ in terms of parameters in the FPLMTO band structure calculation is given in Reference 8. We have suppressed the band index in the following derivation. The band-f Coulomb interaction takes on the form:

$$
J_{n, n^{\prime}}\left(\mathbf{k}, \mathbf{k}^{\prime}\right)=\left\langle\phi_{k}^{*}\left(\mathbf{r}_{1}\right) \psi_{n}^{*}\left(\mathbf{r}_{2}\right)\left|\frac{1}{r_{12}}\right| \psi_{n^{\prime}}\left(\mathbf{r}_{1}\right) \phi_{k^{\prime}}\left(\mathbf{r}_{\mathbf{2}}\right)\right\rangle
$$


Because of the dependence of $J_{n, n^{\prime}}\left(\mathbf{k}, \mathbf{k}^{\prime}\right)$ on $k$ and $k^{\prime}$ there are simply too many elements to be evaluated directly, so we take advantage of the FPLMTO scheme and expand $J_{n, n^{\prime}}\left(\mathbf{k}, \mathbf{k}^{\prime}\right)$ in terms of elements which are independent of $k$ and limited in number. The band wave function $\phi_{k}(\mathbf{r})$ is a linear combination of Bloch basis functions $\Phi_{l m s}^{k}(\mathbf{r})$. Combining equation 2.13 with 2.15 we obtain

$$
\phi_{\mathbf{k}}(\mathbf{r})=\sum_{l m s} A_{l m s}^{\mathbf{k}} \sum_{R} e^{i \mathbf{k} \cdot \mathbf{R}} \Phi_{l m s}(\mathbf{r}-\mathbf{R}),
$$

where $l m s$ are the angular momentum, magnetic quantum number and spin respectively and $R$ is summed over the lattice sites. Note: we have suppressed the sums over $\kappa$ and $\alpha$ because they are given specific values. In the FPLMTO method, $\Phi_{l m s}(r)$ is evaluated at specific energy parameters $E_{l}$ and $\kappa$, which in this calculation is set to the squareroot of the Fermi energy. We also use the approximation that the f-state function $\psi_{n}$ is very small outside of the muffin-tin in which it originated. Thus the contribution to $J_{n, n^{\prime}}\left(\mathbf{k}, \mathbf{k}^{\prime}\right)$ from the integral centered on two-different sites is negligible and therefore we adopt the single-site expansion for the Bloch sums. Splitting the summation over $R$ so that the $R=0$ is the contribution from the muffin-tin orbital centered at the site in question and for all $R \neq 0$ the term becomes the tails of the orbitals when expanded about the site in question gives

$$
\phi_{k}(r)=\sum_{l m s} X_{l m s}^{1}(\mathbf{k}) \Phi_{l m s}^{1}(r)+\sum_{l m s} X_{l m s}^{2}(\mathbf{k}) \Phi_{l m s}^{2}(r),
$$

where $\Phi_{l m s}^{1}(r)$ is the muffin-tin orbital centered at the site in question and the second term represents the tails from all other sites expanded about the site in question. In this case the coefficients $X$ become:

$$
\begin{gathered}
X_{l m s}^{1}(\mathbf{k})=A_{l m s}^{\mathrm{k}} \\
X_{l m s}^{2}(\mathbf{k})=\sum_{l^{\prime} m^{\prime} s^{\prime}} A_{l^{\prime} m^{\prime} s^{\prime}}^{\mathrm{k}} S_{l^{\prime}, m^{\prime}, s^{\prime} ; l, m, s}^{\mathrm{k}},
\end{gathered}
$$

where the $A_{l^{\prime} m^{\prime} s^{\prime}}^{\mathrm{k}}$ are the eigenvectors and $S_{l^{\prime}, m^{\prime}, s^{\prime} ; l, m, s}^{\mathrm{k}}$ are the KKR structure functions. The $J_{n, n^{\prime}}\left(\mathbf{k}, \mathbf{k}^{\prime}\right)$ can be written as a finite sum

$$
J_{n, n^{\prime}}\left(\mathbf{k}, \mathbf{k}^{\prime}\right)=\sum_{t t^{\prime}} \sum_{\substack{l m s \\ l{ }^{\prime} m^{\prime} s^{\prime}}} X_{l m s}^{t *}(\mathbf{k}) B_{n}^{l m s t}{ }_{n^{\prime}}^{l^{\prime} m^{\prime} s^{\prime} t^{\prime}} X_{l^{\prime} m^{\prime} s^{\prime}}^{t^{\prime}}\left(\mathbf{k}^{\prime}\right)
$$


in terms of the coefficients $X_{l m s}^{t}$ and the B tensor, in which the elements are the overlap between the f-states $\psi_{n}(r)$ and the muffin tin orbitals (or MTO tail functions) and is independent of $k$ and $k^{\prime}$, ie.

$$
B_{n}^{l m s t}{ }_{n^{\prime}}^{l m^{\prime} s^{\prime} t^{\prime}}=<\Phi_{l m s}^{t *}\left(r_{1}\right) \psi_{n}^{*}\left(r_{2}\right)\left|\frac{1}{r_{12}}\right| \psi_{n^{\prime}}\left(r_{1}\right) \Phi_{l^{\prime} m^{\prime} s^{\prime}}^{t^{\prime}}\left(r_{2}\right)>.
$$

The FPLMTO calculations already provide a direct calculation of the coefficients and thus only the $B$ tensor needs to be calculated.

For the calculation of the $B$ tensor we separate the $\Phi_{l m s}$ and $\psi_{n}$ into a radial part, angular part, and spin part as

$$
\begin{aligned}
\Phi_{l m s} & =R_{l}(r) Y_{l m}(\theta, \phi) \chi_{s} \\
\psi_{n}(r) & =F_{3}(r)\left[C_{1} Y_{3, n-\frac{1}{2}}(\theta, \phi) \chi_{+\frac{1}{2}}+C_{2} Y_{3, n+\frac{1}{2}}(\theta, \phi) \chi_{-\frac{1}{2}}\right] .
\end{aligned}
$$

Here, the $Y_{l m}$ is the spherical harmonic, $\chi_{s}$ is a spinor, $R_{l}^{t}$ is the radial part of the muffin-tin orbital (or MTO tail), $F_{3}$ is the radial part of the $f$-wave function, and $C_{1}, C_{2}$ are Clebsch-Gordon coefficients. Making use of the addition theorem

$$
\frac{1}{r_{12}}=\sum_{p q} \frac{4 \pi}{2 l+1} \frac{r_{<}^{l}}{r_{>}^{l+1}} Y_{p q}^{*}\left(\theta_{1}, \phi_{1}\right) Y_{p q}\left(\theta_{2}, \phi_{2}\right),
$$

and the relation

$$
\begin{gathered}
\int_{\theta=0}^{\pi} \int_{\phi=0}^{2 \pi} Y_{l m}(\theta, \phi) Y_{l^{\prime} m^{\prime}}(\theta, \phi) Y_{l^{\prime \prime} m^{\prime \prime}}(\theta, \phi) \sin (\theta) d \theta d \phi \\
{\left[\frac{(2 l+1)\left(2 l^{\prime}+1\right)\left(2 l^{\prime \prime}+1\right)}{4 \pi}\right]^{\frac{1}{2}}\left(\begin{array}{ccc}
l & l^{\prime} & l^{\prime \prime} \\
0 & 0 & 0
\end{array}\right)^{\prime}\left(\begin{array}{ccc}
l & l^{\prime} & l^{\prime \prime} \\
m & m^{\prime} & m^{\prime \prime}
\end{array}\right),}
\end{gathered}
$$

we find that

$$
\begin{aligned}
& B_{n}^{l m s t}{ }_{n^{\prime}}^{l^{\prime} s^{\prime} t^{\prime}}=7 *\left[(2 l+1)\left(2 l^{\prime}+1\right)\right]^{\frac{1}{2}} \sum_{p q}(-1)^{m+q+n-\frac{1}{2}}\left(\begin{array}{ccc}
l & p & 3 \\
0 & 0 & 0
\end{array}\right)\left(\begin{array}{ccc}
3 & p & l^{\prime} \\
0 & 0 & 0
\end{array}\right) \\
& \times\left[C_{1}^{n^{\prime}} \delta_{s,+\frac{1}{2}}\left(\begin{array}{ccc}
l & p & 3 \\
-m & -q & n^{\prime}-\frac{1}{2}
\end{array}\right)+C_{2}^{n^{\prime}} \delta_{s,-\frac{1}{2}}\left(\begin{array}{ccc}
l & p & 3 \\
-m & -q & n^{\prime}+\frac{1}{2}
\end{array}\right)\right] \\
& \times\left[C_{1}^{n} \delta_{s^{\prime},+\frac{1}{2}}\left(\begin{array}{ccc}
3 & p & l^{\prime} \\
-\left(n-\frac{1}{2}\right) & q & m^{\prime}
\end{array}\right)-C_{2}^{n} \delta_{s^{\prime},-\frac{1}{2}}\left(\begin{array}{ccc}
3 & p & l^{\prime} \\
-\left(n+\frac{1}{2}\right) & q & m^{\prime}
\end{array}\right)\right]
\end{aligned}
$$




$$
\times \iint R_{l}^{t *}\left(r_{1}\right) R_{l^{\prime}}^{t^{\prime}}\left(r_{2}\right) F_{3}\left(r_{1}\right) F_{3}^{*}\left(r_{2}\right) \frac{r_{<}^{p}}{r_{>}^{p+1}} r_{1}^{2} r_{2}^{2} d r_{1} d r_{2}
$$

Here, we have written the above equations in terms of the $3 \mathrm{j}$ symbols. The ClebschGordon coefficients are $C_{1}^{n}=-\sqrt{\frac{3+\frac{1}{2}-n}{7}}$ and $C_{2}^{n}=\sqrt{\frac{3+\frac{1}{2}+n}{7}}$. We only need to perform the radial integral numerically from the FPLMTO wave functions to calculate the $B$ tensor elements.

All previous derivations have employed a bare Coulomb interaction. To take into account the screening effect of the Coulomb interaction we use

$$
\begin{aligned}
& V(r)=\frac{e^{-r / \lambda}}{r} \\
& \text { with } \\
& \frac{1}{\lambda^{2}}=\frac{6 \pi e^{2} n_{0}}{E_{\text {Fermi }}},
\end{aligned}
$$

where $n_{0}$ is the density of conduction electrons. The addition theorem for the screened interactions becomes

$$
\frac{e^{-r / \lambda}}{r}=\sum_{p q} \frac{4 \pi}{\lambda} \kappa_{p}\left(\frac{r_{>}}{\lambda}\right) i_{p}\left(\frac{r_{<}}{\lambda}\right) Y_{p q}^{*}\left(\theta_{1}, \phi_{1}\right) Y_{p q}\left(\theta_{2}, \phi_{2}\right)
$$

where $i(x)$ and $\kappa(x)$ are the first and second type modified spherical Bessel function. Thus, to include the shielding effects we simply replace the $\frac{r_{<}^{p}}{r_{<}^{p+1}}$ in the radial integral by $\frac{2 p+1}{\lambda} \kappa_{p}\left(\frac{r_{>}}{\lambda}\right) i_{p}\left(\frac{r_{<}}{\lambda}\right)$. The values for $\lambda$ range from $.86 a_{0}$ in CeSb to $1.00 a_{0}$ for CeS. This shielding decreases the size of the $B$ tensors by up to a factor of 2 , depending on the element.

Thus we have the whole calculation of $J_{m m^{\prime}}\left(\mathbf{k}, \mathbf{k}^{\prime}\right)$ by piecing together the coefficients $X$ which fall directly from the FPLMTO band structure calculation with the $B$ overlap tensor which is combination of angular coefficients and a radial integral calculated numerically from the converged FPLMTO potential.

This calculation has been done from a "core" point of view which is valid for the cerium compounds. To be consistent with the treatment of the hybridization in uranium compounds, we would need to adopt a "band" point of view when calculating the Coulomb exchange term for the uranium compounds. However, because of several complications which arise from performing integrals centered on multiple-lattice sites, and the dominance of the hybridization exchange for the uranium compounds, we use the 
"core" point of view to calculate the band-f Coulomb exchange terms for all compounds studied. The implications of this are discussed in the theoretical uncertainties section.

\subsubsection{THE TWO-ION INTERACTION}

Having calculated all of the parameters entering into the Hamiltonian we turn to calculating the two-ion interaction which is responsible for the magnetic ordering. The general form of the two-ion interaction is

$$
H(1,2)=-\sum_{m_{1} m_{1}^{\prime}} \sum_{m_{2} m_{2}^{\prime}} E_{n_{1} m_{1}^{\prime}}^{m_{2} m_{2}^{\prime}}\left(\mathbf{R}_{2}-\mathbf{R}_{1}\right) c_{m_{2}}^{\dagger}(2) c_{m_{2}^{\prime}}(2) c_{m_{1}}^{\dagger}(1) c_{m_{1}^{\prime}}(1)
$$

where $E_{m_{1} m_{1}^{\prime}}^{m_{2} m_{2}^{\prime}}$ is the perturbation energy

$$
E_{m_{1} m_{1}^{\prime}}^{m_{2} m_{2}^{\prime}}\left(\mathbf{R}_{\mathbf{2}}-\mathbf{R}_{\mathbf{1}}\right)=\sum_{i}\left\langle m_{1} m_{2}\left|H_{1} \frac{1}{E_{0}-H_{0}} H_{1} \frac{1}{E_{0}-H_{0}} H_{1} \cdots H_{1}\right| m_{1}^{\prime} m_{2}^{\prime}\right\rangle
$$

Treating the hybridization and exchange Coulomb terms in equation 2.26 to second order in perturbation theory, yields the following result for the two-ion interaction: ${ }^{8}$

$$
\begin{aligned}
E_{m_{a} m_{a}^{\prime}}^{m_{b} m_{b}^{\prime}}\left(\mathbf{R}_{\mathbf{b}}-\mathbf{R}_{\mathbf{a}}\right) & =\sum_{k k^{\prime}} J_{m_{2} m_{2}^{\prime}}\left(\mathbf{k}, \mathbf{k}^{\prime}\right) J_{m_{1} m_{1}^{\prime}}\left(\mathbf{k}^{\prime}, \mathbf{k}\right) e^{-i\left(\mathbf{k}-\mathbf{k}^{\prime}\right) \cdot\left(\mathbf{R}_{2}-\mathbf{R}_{1}\right)} F_{1}\left(\epsilon_{k}, \epsilon_{k^{\prime}}\right) \\
& +\sum_{k k^{\prime}} V_{k^{\prime} m_{2}}^{*} V_{k m_{2}^{\prime}} J_{m_{1} m_{1}^{\prime}}\left(\mathbf{k}^{\prime}, \mathbf{k}\right) e^{-i\left(\mathbf{k}-\mathbf{k}^{\prime}\right) \cdot\left(\mathbf{R}_{2}-\mathbf{R}_{1}\right)} F_{2}\left(\epsilon_{k}, \epsilon_{k^{\prime}}\right) \\
& +\sum_{k k^{\prime}} V_{k^{\prime} m_{2}}^{*} V_{k m_{2}^{\prime}} V_{k m_{1}}^{*} V_{k^{\prime} m_{1}^{\prime}} e^{-i\left(\mathbf{k}-\mathbf{k}^{\prime}\right) \cdot\left(\mathbf{R}_{2}-\mathbf{R}_{1}\right)} F_{3}\left(\epsilon_{k}, \epsilon_{k^{\prime}}\right) \\
& +(1 \leftrightarrow 2)
\end{aligned}
$$

where

$$
\begin{aligned}
F_{1}\left(\epsilon_{k}, \epsilon_{k^{\prime}}\right) & =\frac{1}{\epsilon_{k}-\epsilon_{k^{\prime}}}\left(1-f_{k^{\prime}}\right) f_{k}, \\
F_{2}\left(\epsilon_{k}, \epsilon_{k^{\prime}}\right) & =\left(\frac{-1}{\epsilon_{k^{\prime}}-E_{f}}+\frac{1}{\epsilon_{k}-E_{f}+U}\right) \frac{1}{\epsilon_{k}-\epsilon_{k^{\prime}}}\left(1-f_{k^{\prime}}\right) f_{k} \\
& -\frac{1}{\epsilon_{k^{\prime}}-E_{f}} \frac{1}{\epsilon_{k}-E_{f}}\left(1-f_{k^{\prime}}\right)\left(1-f_{k}\right)-\frac{1}{\epsilon_{k}-E_{f}+U} \frac{1}{\epsilon_{k^{\prime}}-E_{f}+U} f_{k} f_{k^{\prime}}, \\
F_{3}\left(\epsilon_{k}, \epsilon_{k^{\prime}}\right) & =\left[\left(\frac{-1}{\epsilon_{k^{\prime}}-E_{f}}+\frac{1}{\epsilon_{k}-E_{f}+U}\right)^{2} \frac{1}{\epsilon_{k}-\epsilon_{k^{\prime}}}+\frac{2}{U} \frac{1}{\epsilon_{k^{\prime}}-E_{f}} \frac{1}{\epsilon_{k}-E_{f}+U}\right]\left(1-f_{k^{\prime}}\right) f_{k} \\
& +\frac{1}{2} \frac{1}{\epsilon_{k^{\prime}}-E_{f}} \frac{1}{\epsilon_{k}-E_{f}}\left(\frac{2}{U}+\frac{1}{\epsilon_{k}-E_{f}}+\frac{1}{\epsilon_{k^{\prime}}-E_{f}}\right)\left(1-f_{k^{\prime}}\right)\left(1-f_{k}\right) \\
& +\frac{1}{2} \frac{1}{\epsilon_{k^{\prime}}-E_{f}+U} \frac{1}{\epsilon_{k}-E_{f}+U}\left(\frac{2}{U}-\frac{1}{\epsilon_{k^{\prime}}-E_{f}+U}-\frac{1}{\epsilon_{k}-E_{f}+U}\right) f_{k^{\prime}} f_{k},
\end{aligned}
$$


The term $(1 \leftrightarrow 2)$ represents three terms which are the same as the previous ones with atoms indices 1 and 2 . In these equations $f_{k}$ is the Fermi function.

These exchange interactions contain three types of terms: the pure Coulomb exchange interaction term proportional to $J_{m m^{\prime}}^{2}\left(\mathbf{k}, \mathbf{k}^{\prime}\right)$, the hybridization-mediated exchange interaction term proportional to $V_{k m}^{4}$, and the cross term proportional to

$V_{k m}^{2} J_{m m^{\prime}}\left(\mathbf{k}, \mathbf{k}^{\prime}\right)$. These exchange interactions, $E_{m_{a} m_{a}^{\prime}}^{m_{b} m_{b}^{\prime}}\left(\mathbf{R}_{\mathbf{b}}-\mathbf{R}_{\mathbf{a}}\right)$ couple two $f$-ions via single-electron scattering. The matrices calculated generally have $6^{4}$ terms. However, by exploiting the azimuthal symmetry about the interionic axis for free-electron bands, in which case the angular momentum component along $\mathbf{R}_{\mathbf{i j}}$ is conserved by the two-ion interaction, we set $m_{1}=m_{2}^{\prime}$ and $m_{2}=m_{1}^{\prime}$. This reduces the interaction tensor to a much simplier $6 \mathrm{X} 6$ two-ion interaction matrix.

\section{Calculation of the Cross Term}

In this section we discuss the calculation of the term proportional to $V_{k m}^{2} J_{m m^{\prime}}\left(\mathbf{k}, \mathbf{k}^{\prime}\right)$ in the two-ion interaction. The other two terms are calculated in similar fashions but are not as complex as this one. Thus, we provide a more detailed explanation of it.

The cross term interaction in equation 2.54 is

$$
E_{V^{2} J}=\sum_{k k^{\prime}} V_{k^{\prime} m_{2}}^{*} V_{k m_{2}^{\prime}} J_{m_{1} m_{1}^{\prime}}\left(\mathbf{k}^{\prime}, \mathbf{k}\right) e^{-i\left(\mathbf{k}-\mathbf{k}^{\prime}\right) \cdot\left(\mathbf{R}_{2}-\mathbf{R}_{1}\right)} F_{2}\left(\epsilon_{k}, \epsilon_{k^{\prime}}\right)
$$

Substituting the form of the band-f exchange term given in equation 2.43 , in which we have split up the sum over coefficients and an overlap tensor, into the above equation this cross term becomes

$$
E_{V^{2} J}=\sum_{\alpha, \alpha^{\prime}} B_{m_{1}}^{\alpha}{\stackrel{\alpha^{\prime}}{m_{1}^{\prime}}}_{k k^{\prime}} V_{k^{\prime} m_{2}}^{*} V_{k m_{2}^{\prime}} X_{\alpha}\left(\mathbf{k}^{\prime}\right) X_{\alpha^{\prime}}^{*}(\mathbf{k}) e^{-i\left(\mathbf{k}-\mathbf{k}^{\prime}\right) \cdot\left(\mathbf{R}_{2}-\mathbf{R}_{1}\right)} F_{2}\left(\epsilon_{k}, \epsilon_{k^{\prime}}\right)+(1 \leftrightarrow 2) .
$$

Here, $\alpha$ indicates the FPLMTO quantum numbers $l m_{l} s$ and the radial function type $t$ described in the previous section on the calculation of the Coulomb exchange term. Next, using the tetrahedral method, the summations over $k$ and $k^{\prime}$ are converted into integrals over energy. Choosing $\mathbf{R}_{1}=\mathbf{0}$ to be a central site, the term is then calculated 
for the first, second, and third nearest neighbor shells, corresponding to specific choices of $\mathbf{R}_{\mathbf{2}}$ and is quantized along the interatomic axis $\mathbf{R}=\mathbf{R}_{\mathbf{2}}-\mathbf{R}_{\mathbf{1}}$. To understand this step, recall that we calculate all quantities entering into the model Hamiltonian using an $89 \mathrm{k}$-point tetrahedral mesh in the irreducible Brillouin zone quantized along the [001] direction. First, we perform a rotation of all quantities from the [001] axis to the interatomic axis. This rotation must be performed for all quantities which depend upon the quantization axis, ( $X$ and $V$ as well as the overlapping tensor $B$ ). To perform the integration over the entire Brillouin zone, we rotate the k-point mesh using the 48 point group operations of the full cubic symmetry, as well as rotating the individual quantities which depend on $\mathbf{k}$ point ( $X$ and $V$ ). It is convenient to introduce the intermediate quantity,

$$
A_{\alpha m}(\epsilon, R)=\frac{1}{N(\epsilon)} \frac{\Omega}{(2 \pi)^{3}} \sum_{N} \int d^{3} k V_{k m}^{n *} X_{\alpha}^{n}(\mathbf{k}) e^{i \mathbf{k} \cdot \mathbf{R}} \delta\left(\epsilon-\epsilon^{n}(\mathbf{k})\right),
$$

where $\Omega$ is the unit cell volume, $n$ is the band index which has been suppressed in previous equations, and the function $N(\epsilon)$ is the density of states. In the calculation of the hybridization exchange interaction, the analogous quantity is ${ }^{12}$

$$
V_{m m^{\prime}}(\epsilon, R)=\frac{1}{N(\epsilon)} \frac{\Omega}{(2 \pi)^{3}} \sum_{n} \int d^{3} k V_{m k}^{N *} V_{m^{\prime} k}^{N} e^{i \mathbf{k} \cdot \mathbf{R}} \delta\left(\epsilon-\epsilon^{(N)}(\mathbf{k})\right)
$$

This equation represents a general form of the two-ion interaction. However, when exploiting the azimuthal symmetry mentioned above, we only need to take into account interactions which conserve magnetic quantum number $m$. This is equivalent to setting $m$ equal to $m^{\prime}$ in the previous equation and the defined quantity becomes real. A similar scheme is used for the pure Coulomb exchange interaction. However, it is evident from the above equation for the cross term interaction that no such simplification is available at this point in the calculation. We then continue to write the cross term contribution to the two-ion interaction as

$$
E_{V^{2} J}(\mathbf{R})=\sum_{\alpha \alpha^{\prime}} B_{m_{1} m_{1}^{\prime}}^{\alpha \alpha^{\prime}} \int d \epsilon^{\prime} \int d \epsilon N(\epsilon) A_{\alpha m_{2}}^{*}\left(\epsilon^{\prime}\right) A_{\alpha^{\prime} m_{2}^{\prime}}(\epsilon) F_{2}\left(\epsilon, \epsilon^{\prime}\right)+(1 \leftrightarrow 2)
$$

The two-ion interaction matrix $E$ is an energy and must be a real quantity for all values of $m_{1}, m_{1}^{\prime}, m_{2}, m_{2}^{\prime}$ and $R$. In previous calculations, this term was assumed to be real 
and calculated as such. However, here we examine it more explicitly. The symmetry between atoms 1 and 2 and the summation over $\alpha \alpha^{\prime}$ ensures that this quantity will be real provided that the integrals over $\epsilon$ and $\epsilon^{\prime}$ are equivalent. In principle, this is obvious. However in the form of the function $F_{2}$ in equation 2.54 it is not the case for the term which is proportional to $\left(1-f_{k^{\prime}}\right) f_{k}$. Thus, to be technically correct in this calculation we replace $F_{2}\left(\epsilon, \epsilon^{\prime}\right)$ with $F_{2}=\frac{1}{2}\left(F_{2}\left(\epsilon, \epsilon^{\prime}\right)+F_{2}\left(\epsilon^{\prime}, \epsilon\right)\right)$. This only affects the term proportional to $\left(1-f_{k^{\prime}}\right) f_{k}$ which is not already symmetric with respect to the interchange of $k$ and $k^{\prime}$. Note, that this change only affects the complex part of $E_{V^{2} J}$ making it vanish. After performing the summations and integrations we exploit the azimuthal symmetry along the interatomic axis and set $m_{1}=m_{2}^{\prime}$ and $m_{1}^{\prime}=m_{2}$ conserving angular quantum number and obtaining the $6 \times 6$ cross term contribution to the two-ion interaction matrix.

\section{Two-Ion Interaction of the $f^{3}$ Configurations in the Uranium Compounds}

The previous calculations obtained the two-ion interaction matrices coupling two $f$ electrons via single-electron scattering. However, we cannot simply neglect the interactions among the three on-site $f$ electrons of the uranium compounds. These electrons form $f^{3}$ multiplets. Therefore, we need to obtain the two-ion interactions between the $f^{3}$ multiplets which can be determined in terms of the one-electron interactions. ${ }^{14}$ Keeping only terms to lowest order in perturbation results in

$$
\mathcal{E}_{N M}^{M N}\left(\mathbf{R}_{\mathbf{b}}-\mathbf{R}_{\mathbf{a}}\right)=\sum_{m n} P_{m \rightarrow n}^{J M \rightarrow J N} P_{n \rightarrow m}^{J N \rightarrow J M} E_{n m}^{m n}\left(\mathbf{R}_{\mathbf{b}}-\mathbf{R}_{\mathbf{a}}\right) .
$$

Here the $P_{m \rightarrow n}^{J M \rightarrow J N}$ are the transition rates of an $f^{3}$ multiplet from a state $J M$ to state $J N$, under the restriction that an $f$ electron can be created and annihilated only once as its state changes from $m$ to $n$. These transition rates are obtained by constructing the multiplet wave function $\Psi_{J M}(1,2,3)$. We use the LS coupling of single electron states. The ground-state $f^{3}$ multiplet has a total spin $S=\frac{3}{2}$, a total orbital angular momentum $L=6$, and a total angular moment $J=\frac{9}{2}$. The details of this calculation can be found in reference 14 . In this fashion we obtain a 10X10 two-ion interaction matrix coupling the $f_{9 / 2}^{3}$ multiplets of neighboring uranium sites. 


\subsubsection{THE MEAN FIELD CALCULATION}

With the two-ion interactions having been determined, the ordering temperature and zero temperature ordered moment can be determined by means of a mean field calculation. ${ }^{9}$ The two-ion Hamiltonian is

$$
H=-\sum_{i, j} \sum_{\mu, \nu} \sum_{\epsilon, \sigma} \xi_{\mu \nu}^{\epsilon \sigma}\left(\theta_{i j}\right) e^{-i(\mu-\nu+\epsilon-\sigma) \phi_{i j}} \mathbf{J}_{\mu \nu}^{i} \mathbf{J}_{\epsilon \sigma}^{j}
$$

where

$$
\xi_{\mu \nu}^{\epsilon \sigma}(\theta)=\sum_{m_{1}, m_{1}^{\prime}} \sum_{m_{2}, m_{2}^{\prime}} E_{m_{1} m_{1}^{\prime}}^{m_{2} m_{2}^{\prime}}\left(\mathbf{R}_{\mathbf{i j}}\right) \times D_{m, \alpha}^{(J)}(\theta)
$$

Here, $i$ and $j$ label cerium (uranium) sites, $\theta_{i j}$ and $\phi_{i j}$ are the angular coordinates of the interionic axis $\mathbf{R}_{\mathbf{i j}}$ with respect to the axis of quantization which we have chosen along the $<001>$ direction for convenience. The $\mu, \nu, \epsilon, \sigma$ are single-ion states of the $z$ component of the angular momentum, $\left(J=\frac{5}{2}\right)$ for cerium compounds and $\left(J=\frac{9}{2}\right)$ for uranium compounds, quantized along the chosen direction. Here $D$ is the product of four rotation matrices transferring the exchange parameters from the interatomic axis, in which they were calculated, to the chosen axis of quantization. We have used $m$ and $\alpha$ to represent the dependence of $D$ on all $m$ values and all single-ion states thus providing an appropriate rotation tensor for the two-ion interactions parameters $\mathrm{E}(\mathbf{R})$. In fact, we can simply think of these $\xi$ entering the hamiltonian as rotations of the previously calculated two-ion interaction matrix; from the interatomic axis, in which the exchange parameters are calculated, to the choice for the quantization axis. A full expression for $\xi$ with the $D(\theta)$ written out explicitly can be found in reference 9 . In the hamiltonian equation the standard basis operators, $J_{\mu \nu}^{i} \equiv|\mu\rangle\langle\nu|$ transfer the Ce (U) ion site $i$ from the state $|\nu\rangle$ to the state $|\mu\rangle$. In this way, we obtain the hamiltonian from which to calculate the magnetic parameters using the mean field approximation.

Beginning at zero temperature, the magnetic system in question is converged and the ground state magnetic properties are extracted. Then the temperature is slowly increased and the ordered ground state of the system is recalculated and its energy compared to that of the paramagnetic state. This process is continued until either the energy of the ordered ground state exceeds that of the paramagnetic state or the 
magnetic moment of the ordered ground state vanishes. Both of these indicate that paramagnetic state has the lowest energy at that temperature, thus giving the ordering temperature. In this way we calculate both the zero temperature ordered moment and the ordering temperature across both the cerium and uranium compound series. We also compare the energetics to determine the ground state configurations of the systems according to this model hamiltonian approach.

\section{THE CRYSTAL FIELD INTERACTION FOR CERIUM COMPOUNDS}

We now discuss the need to include for the first time the crystal field interaction term in the model Hamiltonian of the cerium compounds, so we can investigate the interplay of the exchange and crystal field interactions on an equal footing as we chemically tune the various pertinent interactions across the series. First, note that in the model hamiltonian itself we have approximated all $f$ states of the $j=\frac{5}{2}$ manifold of the cerium compounds to have the same energy $E_{f}$. Therefore, we do not account for the crystal field splitting directly in the exchange interaction hamiltonian. Also, it is important to note that when calculating the parameters entering into the model hamiltonian in this approach, the $4 f$ states of cerium are treated as "core" states, and thus, only interact with the spherical component of the effective one-electron potential. Thus, the interaction of the atomic like $4 f$ states with the non-spherical components of the potential, which give rise to the crystal field splitting, is not explicitly included. This is the case for both the warped muffin tin calculations ${ }^{8}$ and the current full potential calculations. Therefore, these crystal field interactions are never taken into account, either directly through multiple $4 f$ state energies in the model hamiltonian $\left(E_{f}\right.$ is single valued), or indirectly in

the interactions themselves from the FPLMTO potential ( $4 f$ states placed in the core). Thus, it is necessary to include a separate crystal field splitting interaction in the model Hamiltonian on an equal footing with the exchange interactions. For cerium compounds, we include the crystal field hamiltonian. ${ }^{13}$

$$
H_{C F}=B_{4}\left(O_{4}^{0}+5 O_{4}^{4}\right)
$$


where

$$
\begin{aligned}
O_{4}^{0} & =35 J_{z}^{4}-30 J(J+1) J_{z}^{2}+25 J_{z}^{2}-6 J(J+1)+3 J^{2}(J+1)^{2} \\
O_{4}^{4} & =\frac{1}{2}\left(J_{+}^{4}+J_{-}^{4}\right)
\end{aligned}
$$

are the Stevens equivalence operators. The coefficient

$$
B_{4}=\frac{7}{16} \frac{Z e^{2}}{R^{5}} \cdot<r^{4}>\beta
$$

is a function of $\left\langle r^{4}\right\rangle$, determines the scale of the crystal field splittings. Here $\beta$ is the Stevens multiplicative factor. ${ }^{16}$ Note that we have omitted the term involving $\left\langle r^{6}\right\rangle$ from $\left(H_{C F}\right)$ because it has no effect on the $j=\frac{5}{2}$ manifold present in these systems. These operators give us the following hamiltonian.

$$
\left\langle J_{z}\left|H_{C F}\right| J_{z}^{\prime}\right\rangle=60 B_{4}\left(\begin{array}{cccccc}
1 & 0 & 0 & 0 & \sqrt{5} & 0 \\
0 & -3 & 0 & 0 & 0 & \sqrt{5} \\
0 & 0 & 2 & 0 & 0 & 0 \\
0 & 0 & 0 & 2 & 0 & 0 \\
\sqrt{5} & 0 & 0 & 0 & -3 & 0 \\
0 & \sqrt{5} & 0 & 0 & 0 & 1
\end{array}\right)
$$

Diagonalizing this hamiltonian one finds that the two-fold degenerate states $\left|\Gamma_{7}\right\rangle=$ $\sqrt{\frac{1}{6}}\left| \pm \frac{5}{2}\right\rangle-\sqrt{\frac{5}{6}}\left|\mp \frac{3}{2}\right\rangle$ have an energy $E_{\Gamma_{7}}=-240 B_{4}$ and the four-fold degenerate states $\left|\Gamma_{8}\right\rangle=\sqrt{\frac{5}{6}}\left| \pm \frac{5}{2}\right\rangle+\sqrt{\frac{1}{6}}\left|\mp \frac{3}{2}\right\rangle$ and $\left|\Gamma_{8}\right\rangle=\left| \pm \frac{1}{2}\right\rangle$ have an energy $E_{\Gamma_{8}}=+120 B_{4}$. Here the quantity, $B_{4}$ is taken to be positive when the $\Gamma_{7}$ splitting is the ground state. In these cases we find that the crystal field splitting is $\Delta_{C F}=E_{\Gamma_{8}}-E_{\Gamma_{7}}=360 B_{4}$. In the absence of an $a b$ initio value of the CF interaction in this class of strongly correlated electron systems ${ }^{17}$ the $C F$ splitting, $\Delta_{C F}=360 B_{4}$, is set to the experimental values ${ }^{18-21}$, listed in Table 2.3.

This term is then added to the mean field hamiltonian, equation 2.60 and the zerotemperature ordered moment and ordering temperature are calculated by mean field calculations as describe before.

Note that we are unable to use this type of crystal field term in the uranium compound calculations because (1) technical difficulties involved in the treatment of the 
crystal field splitting of the $j=9 / 2$ manifold, (2) partial inclusion of the crystal field splitting in the band point of view treatment for the $5 f$ states in these compounds, and (3) a lack of experimental results. This is discussed further in chapter 4.

Table 2.3: Experimental values of the crystal field splitting for the cerium compounds given in Kelvin units.

$\Delta_{C F}(\mathrm{~K})$\begin{tabular}{|c|c|c|c|c|c|c|}
$\mathrm{CeS}$ & $\mathrm{CeSe}$ & $\mathrm{CeTe}$ & $\mathrm{CeP}$ & $\mathrm{CeAs}$ & $\mathrm{CeSb}$ & $\mathrm{CeBi}$ \\
140 & 116 & 32 & 150 & 137 & 37 & 8 \\
\hline
\end{tabular}




\subsection{THEORETICAL UNCERTAINTIES}

In this section we discuss the sensitivity of the calculations to certain parameters involved and the overall uncertainty in the final results obtained. First, recall that for the pure FPLMTO spin-polarized electronic structure calculations only the zero-temperature ordered moments and easy direction are calculated. In the model hamiltonian method, we not only calculated the ordered moment, but also the ordering temperature as well as determining the ground state configuration. This section addresses the repeatability of the results and not the accuracy. Also note that these are only estimates of the uncertainty and not specific systematic studies.

One type of uncertainty is the sensitive nature of these calculations to certain parameters used during the computation. These are calculated by simply changing a parameter and noting the difference in the final results obtained. In all cases, we note that the parameters in the calculations presented in this paper were chosen to best represent the theory regardless of the results obtained. Often, there may be several reasonable choices of a parameter used in the calculations of the magnetic properties of the compounds studied. In other cases, however, we must provide a more precise definition of what is the best choice of a parameter based on the physical principles involved.

Other uncertainties come as a direct result of approximations which we have made in the derivation. These are calculated by first estimating the uncertainty in some intermediate result caused by the approximation. Then changing the intermediate result and recalculating the final answer and noting the difference.

\subsubsection{UNCERTAINTY IN FPLMTO METHOD}

The easily repeatable FPLMTO electronic structure calculations, either with or without orbital polarization, have very little uncertainty involved with the choice of the parameters used to converge these potentials.

The largest variance in calculation of the ordered moments for these compounds comes from the method used in choosing a muffin-tin radius. These purely mathematical constructs are non-physical and approximate a "boundary" between the area of the 
potential dominated by a particular lattice site and either an area dominated by another lattice site, or the "interstitial" area not dominated by any one lattice site configuration. In general, these are chosen to be "touching" in order to fill up the most possible volume. Reasonable choices of muffin-tin radius would include taking ratios of the atomic sizes of the atoms in the crystal, varying the muffin-tin radii until a lowest energy configuration is found, or even simply dividing the space evenly among the lattice sites regardless of nuclear charge. For these calculations, however, the uncertainty of the choice in muffin-tin radius can be offset if one converges the potential with higher cutoff in the parameters like $l_{m a x}, G_{b m a x}$ etc. Regardless, the uncertainly in this calculation with reguard to the ordered moment is very small and through comparisons among various choices of parameters is found to be $\delta_{\mu_{0}}= \pm 0.05 \mu_{B}$.

The converged energies are more affected by the above, however, in determining the direction of anisotropy the difference in energy of multiple configurations are compared. Since all configurations of a particular compound are calculated using the same muffintin radii, the differences are affected only slightly. Therefore, the uncertainty in the calculated energies of anisotropy using this method are $\delta_{\Delta E}= \pm 5 m R y$ for the uranium compounds and $\delta_{\Delta E}= \pm .3 m R y$ for the cerium compounds. As long as reasonable choices of muffin-tin radii and a sufficient number of k-points are used in the convergence, the results are not sensitive to the choices made.

\subsubsection{UNCERTAINTY IN THE MODEL HAMILTONIAN METHOD}

Because there are two types of calculations for the two classes of compounds, we must discuss the uncertainty important to each one.

\section{A. Uncertainty in Uranium Magnetic Properties}

Here we will address two major concerns. First, is the calculation of the band-f coulomb

exchange for the uranium compounds using the "core" point of view and second, the sensitivity of the hybridization parameters to the choice of $\kappa$ used in the FPLMTO electronic structure calculation. 
Table 2.4: Characteristic matrix elements of the $10 \times 10$ two-ion interaction matrix $\left(E_{m m^{\prime}}^{m^{\prime} m}(R)\right)$ of the $f^{3}$ multiplet for uranium sulfide. Values for the hybridization induced $\left(E_{V^{4}}\right)$, cross terms $\left(E_{V^{2} J}\right)$, and pure Coulomb exchange $\left(E_{J^{2}}\right)$ interactions are listed for the $m=m^{\prime}=\frac{5}{2}$ elements for each of the first, second, and third nearest neighbors in units of $\mathrm{K}$.

\begin{tabular}{|l|c|c|c|}
\hline & $E_{V^{4}}$ & $E_{V^{2} J}$ & $E_{J^{2}}$ \\
\hline $\mathrm{R}=\left(\begin{array}{lll}\frac{1}{2} & \frac{1}{2} & 0\end{array}\right)$ & 253 & 14.4 & 2.0 \\
\hline $\mathrm{R}=\left(\begin{array}{lll}1 & 0 & 0\end{array}\right)$ & 490 & 12.9 & 1.0 \\
\hline $\mathrm{R}=\left(\begin{array}{lll}1 & \frac{1}{2} & \frac{1}{2}\end{array}\right)$ & 29 & 4.2 & 0.1 \\
\hline
\end{tabular}

Table 2.4 shows the characteristic matrix elements of the $10 \times 10$ two-ion interaction matrix of the $f^{3}$ multiplet of uranium sulfide, a typical uranium compound. It is easily noted that the pure Coulomb exchange interaction is very small compared to the dominant hybridization exchange interaction. The uncertainty, therefore, is mainly in the effect this quantity has on the cross interaction. Calculating the band-f exchange from a "band point of view" would increase these terms for the lighter compounds, such as uranium sulfide, but actually decrease these terms for the heavier compounds as noted in earlier. Since no previous calculation of the band-f Coulomb exchange from the "band point of view" exists, and to demonstrate the validity of our method, we use a generous estimate of the uncertainty in the band-f Coulomb exchange term to be $50 \%$ of the calculated quantity from the "core" point of view. However, even this large uncertainty in the Coulomb exchange interactions, only increase or decrease the cross terms by a factor of $\sqrt{1.5}=1.22$. Since the total two-ion interaction is obtained from adding the three contributions together this is only a $1 \%$ effect overall.

We have found that the values of the exchange parameters are extremely sensitive to the parameter $\kappa$ used in the FPLMTO calculations. Small changes in this parameter which we use to determine the tails of both the $f$ and non- $f$ wave functions when calculating the hybridization, creates large differences in the results for the two-ion hybridization exchange matrix, and thus the ordering temperatures are likewise affected. Depending 
upon the method used to chose this parameter, one can obtain a wide range of results. For example, using different seemingly reasonable methods to choose $\kappa$ give ordering temperatures of uranium sulfide which range from $450 \mathrm{~K}$ to $1200 \mathrm{~K}$ ! For this reason we must first use physical principles to determine the method of choosing this parameter which best represents the hybridization of the wave functions in question. We choose $\kappa^{2}$ to be the center of the occupied $f$ valence band when computing the hybridization parameters. This is the best choice of $\kappa$ because the wave functions are best represented in the center of where the hybridization actually takes place. However, even with this specific constraint we are still faced with the fact that the exchange interactions are extremely sensitive to this number in the region surrounding the center of the occupied $f$-band. Tedious calculations have been able to reduce the uncertainty in the central- $f$ energy to about $5 \mathrm{mRy}$, but this still produces about a $20 \%$ uncertainty in the size of the exchange parameters. Since the ordering temperature from the mean field calculation scales on the order of the exchange parameters entering therein, the ordering temperatures for the uranium compounds have an overall uncertainty of about $20 \%$. The ordered moments, however, are not affected by this scaling of the exchange parameters and are only influenced by the interaction among the off-diagonal matrix elements which mix the states and therefore the uncertainty in this quantity is minimal even though the size of the hybridization exchange interactions varies a great deal.

\section{B. Uncertainty in Cerium Magnetic Properties}

Unlike the uranium compounds, the FPLMTO parameters do not produce such large uncertainties in the magnetic calculations of the cerium compounds. Because the $f$ electrons are placed into the "core" and treated as atomic like, these parameters become very well defined. Overall, the uncertainty associated with the choice of FPLMTO parameters is approximately $5 \%$ in the size of the exchange interactions.

A much greater contribution to the uncertainty is the size of the crystal field parameters entering into the mean field hamiltonian. These parameters are taken from experimental results of the crystal field splitting, which in themselves, have uncertainty. Depending upon the size of the crystal field in comparison to the calculated exchange 
induced interactions the uncertainty in the ordered moment varies from compound to compound. By varying the amount of crystal field added to the hamiltonian by the experimental uncertainty and examining the effect on the calculated ordered moments, we obtain a good estimate of the uncertainty in the ordered moments of the cerium compounds. Further discussion and a listing of this uncertainty along with of the calculated ordered moments for the cerium compounds is presented along with the results in chapter 3. The ordering temperature is less affected by this parameter: for the lighter compounds because the crystal field already dominates, and for the heavier compounds because the exchange interactions dominate. 


\subsection{SUMMARY}

To summarize, we have performed $a b$ initio based calculations of the magnetic properties using two basic approaches.

First, we utilize a spin-polarized full potential linear muffin tin orbital electronic structure calculations both with and without orbital polarization correction. From this calculation we obtain the zero temperature ordered moment and by comparing energetics the direction of anisotropy.

Second, we have formulated a synthesis of the phenomenology based on the Anderson and Kondo Hamiltonian which we have derived from first principles treating the hybridization exchange interaction and band-f Coulomb exchange interactions on an equal footing. Using FPLMTO electronic structure calculations, we obtain a first principles evaluation of the parameters entering this hamiltonian. By formulating two different treatments of the $f$ electrons, "core" and "band" points of view, we properly treat both the more "atomic like" cerium compounds as well as the more "band like" uranium compounds. For the cerium compounds, we have also performed a first time calculation where we have also incorporated the crystal field effects on an equal footing with the exchange interactions. Using the mean field approximation, we then determine the zero-temperature ordered moment, the ordering temperature, and the ground state configurations of all of the cerium and uranium monopnictides and monochalcogenides. 


\section{Chapter 2 References}

1. P. Fulde, Electron Correlations in Molecules and Solids (Springer-Verlag, 1991).

2. Vosko et. al., Can. J. Phys. 58, 1200 (1980).

3. H. L. Skriver, The LMTO Method (Springer, Berlin, 1984).

4. D. L. Price and B. R. Cooper, Phys. Rev. B 39, 4945 (1989).

5. G. P. Srivastava, J. Phys. A 17, 1317(1984).

6. O. Eriksson, M. S. S. Brooks, and B. Johansson, Phys. Rev. B 41, 7311 (1990).

7. H. J. Monkhorst and J. D. Pack, Phys. Rev. B 13, 5188 (1976).

8. Q. G. Sheng and B. R. Cooper, J. Appl. Phys. 69, 5472 (1991); Phys. Rev. B 50,965 (1994).

9. N. Kioussis, B. R. Cooper, and J. M. Wills, Phys. Rev. B 44, 10,003 (1991); J. Appl. Phys. 633683 (1988).

10. N. Kioussis, H. J. Yu, B. R. Cooper, Q. G. Sheng, and J. M. Wills, J. Appl. Phys. 73,5424 (1993).

11. M. R. Norman, D. D. Koelling, A. J. Freeman, H. J. F. Jansen, B. I. Min, T. Oguchi, and Ling Ye, Phys. Rev. Lett. 53, 1673 (1984).

12. J. M. Wills and B. R. Cooper, Phys. Rev. B 36, 3809 (1987).

13. N. Kioussis, D. Swearingen, B. R. Cooper, and J. M. Wills, J. Appl. Phys. 69 5475 (1991).

14. Q. G. Sheng, B. R. Cooper, and S. P. Lim, Phys. Rev. B 509215 (1994).

15. K. R. Lea, M. J. M. Leask, and W. P. Wolf, J. Phys. Chem. Solids 23, 1381 (1962).

16. K. W. H. Stevens, Proc. phys. Soc. Lond. A65, 209 (1952). 
17. N. Kioussis (private communication).

18. A. Dönni, A. Furrer, P. Fischer and F. Hulliger, Physica B 186-188, 541 (1993).

19. F. Hulliger, B. Natterer, H. R. Ott, J. Mag. Magn. Mat. 8, 87 (1978).

20. J. Rossat-Mignod, P. Burlet, S. Quezel, J. M. Effantin, D. Delacôte, H. Bartholin, O. Vogt, and D. Ravot, J. Magn. Magn. Mater. 31-34, 398 (1983).

21. G. Gilat and N. R. Bharatiya, Phys. Rev. B 12, 3479 (1975). 


\section{Chapter 3}

\section{RESULTS ON CERIUM COMPOUNDS}

In this chapter we discuss the results from the two $a b$ initio based calculations of the magnetic properties for the cerium compounds. We find that while the pure LDA calculation fails, even qualitatively, to obtain agreement with experiment, the calculations using the model hamiltonian method including hybridization, Coulomb exchange, and the crystal field give excellent agreement with the experimental results for the ordered moment and ordering temperature across the entire series of cerium compounds. 


\subsection{LDA RESULTS}

The spin polarization of the FPLMTO calculations aligns the spins of the electrons along a chosen direction. Shown in Figures 3.1 through 3.4 are contour plots of the spin-magnetization density, $\rho_{\downarrow}-\rho_{\uparrow}$ for CeP contrasting the effects of the directions of polarization as well as the behavior of the spin charge density in the planes parallel to the polarization and perpendicular to the polarization. Figures 3.1 and 3.2 show spinmagnetization density for polarization in the $<001>$ direction while Figures 3.3 and 3.4 display spin-magnetization density for polarization in the $<111>$ direction. Note that in the presence of spin polarization cubic symmetries pertaining to rotations about the polarization axis are retained, as seen in the figures of the perpendicular planes (Figures 3.2 and 3.4). Looking at the planes parallel to the polarization (Figures 3.1 and 3.3) we can see a distortion of the spin-magnetization density, just as we expect. Also note that even in the direction of polarization the system maintains the symmetry of reflection about the plane perpendicular to the polarization at the origin and time reversal symmetry. In other words, the choice of spin "up" and spin "down" are completely arbitrary. If all spins and moments are reversed, then the system has exactly the same energy. More importantly note that the phosphorus atom sites have little or no spin magnetization and does not contribute directly to the magnetic properties of this system. This is true for all the compounds we have studied. The differences in the magnetic properties between in these compounds must therefore arise from the effects of the ligands on the cerium sites.

Orbital polarization shifts the energy levels of the different $m_{l}$ states of the system, thus favoring certain values of $m_{l}$ over others. The scaling of the energy shifts are from the self consistently calculated Racah parameters given in Table 3.1.

The effect of orbital polarization on the spin moment of the cerium $f$ states is shown in Figures 3.5 through 3.8. These plots show the $f$-projected density of states for spin up and spin down. Plots are shown both with and without orbital polarization taken into account for a typical pnictide system (CeP) and a typical chalcogenide system (CeS). These plots show the spreading of the spin-down (up) states favoring particular values of 
Figure 3.1: Spin magnetization density $\rho_{\downarrow}-\rho_{\uparrow}$ for CeP in the plane parallel to the spin polarization direction $<001>$. Solid lines indicate higher spin down density, dashed lines indicate higher spin up density.

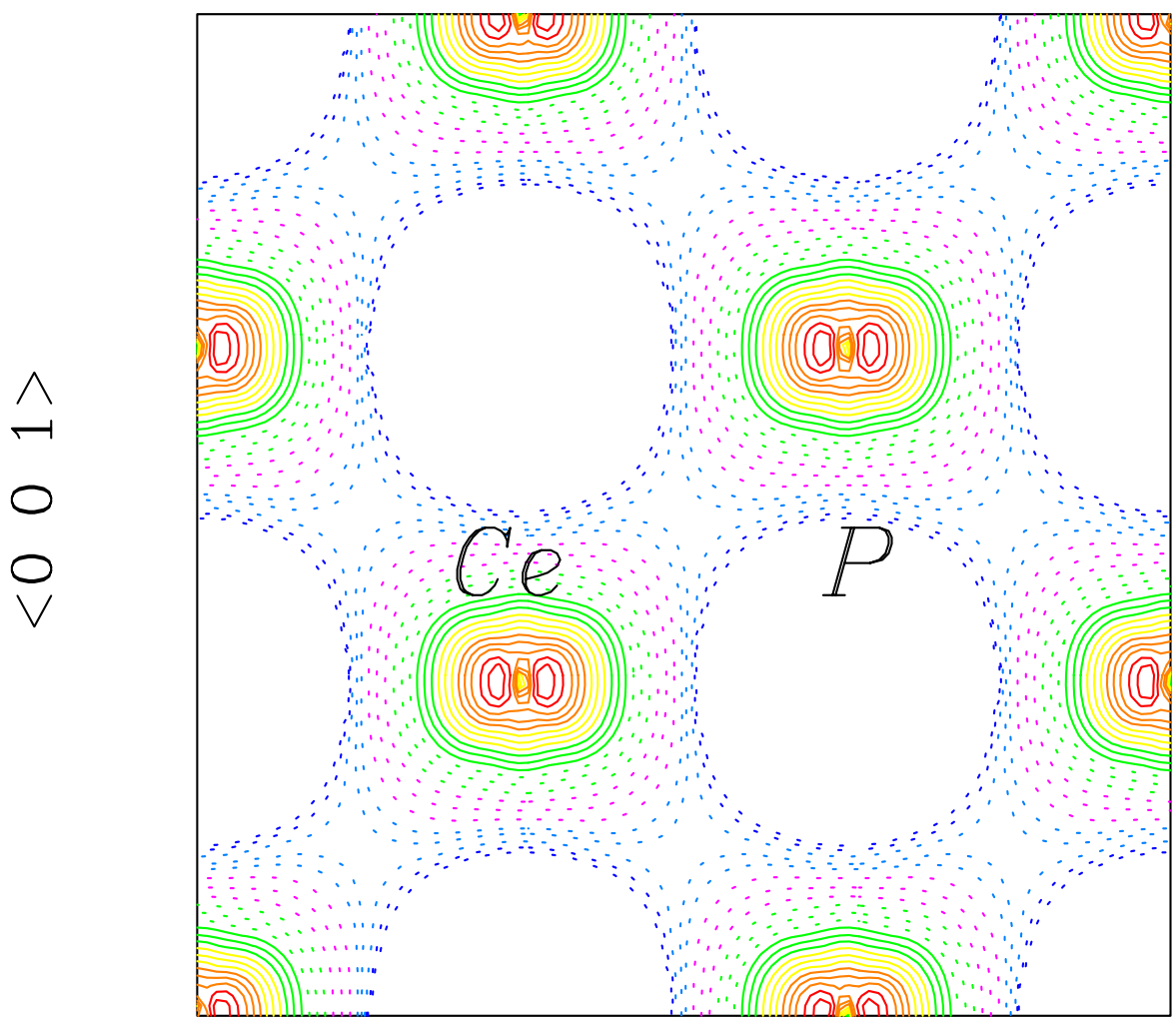

$<1 \quad 0 \quad 0>$ 
Figure 3.2: Spin magnetization density $\rho_{\downarrow}-\rho_{\uparrow}$ for $\mathrm{CeP}$ in the plane perpendicular to the spin polarization direction $<001>$. Solid lines indicate higher spin down density, dashed lines indicate higher spin up density.

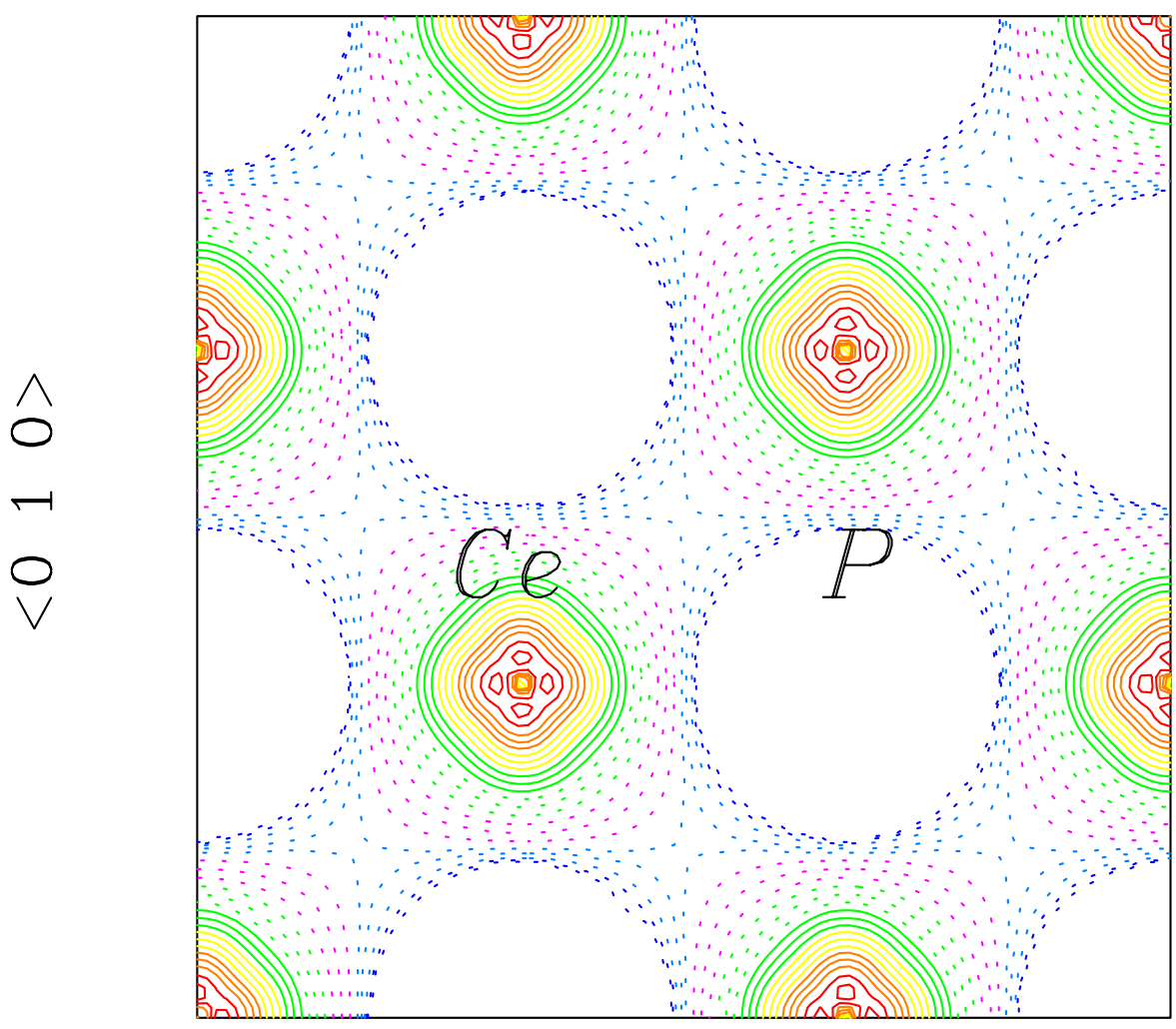

$<\begin{array}{lll}1 & 0 & 0\end{array}>$ 
Figure 3.3: Spin magnetization density $\rho_{\downarrow}-\rho_{\uparrow}$ for CeP in the plane parallel to the spin polarization direction $<111>$. Solid lines indicate higher spin down density, dashed lines indicate higher spin up density.

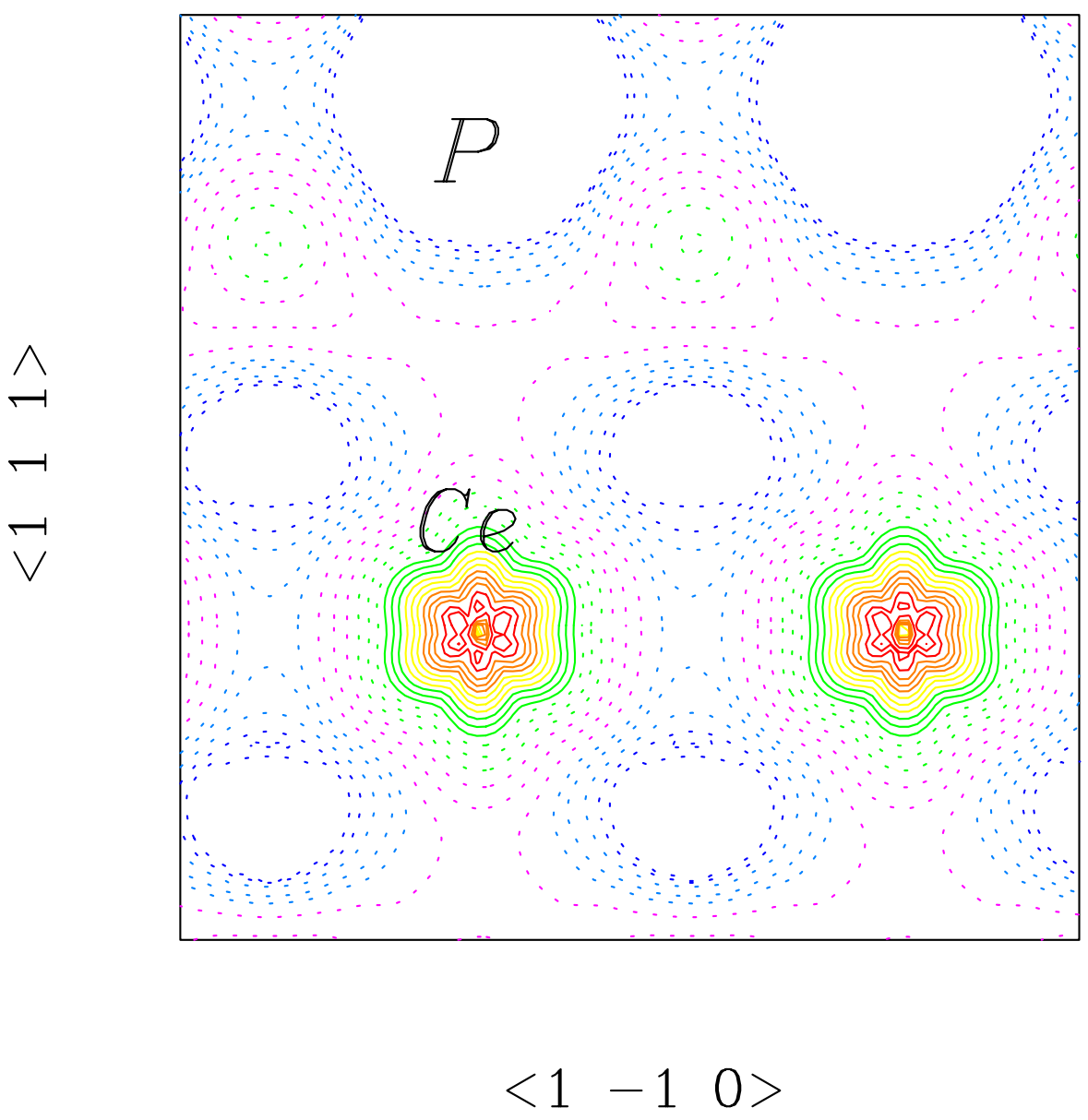


Figure 3.4: Spin magnetization density $\rho_{\downarrow}-\rho_{\uparrow}$ for $\mathrm{CeP}$ in the plane perpendicular to the spin polarization direction $<111>$. Solid lines indicate higher spin down density, dashed lines indicate higher spin up density.

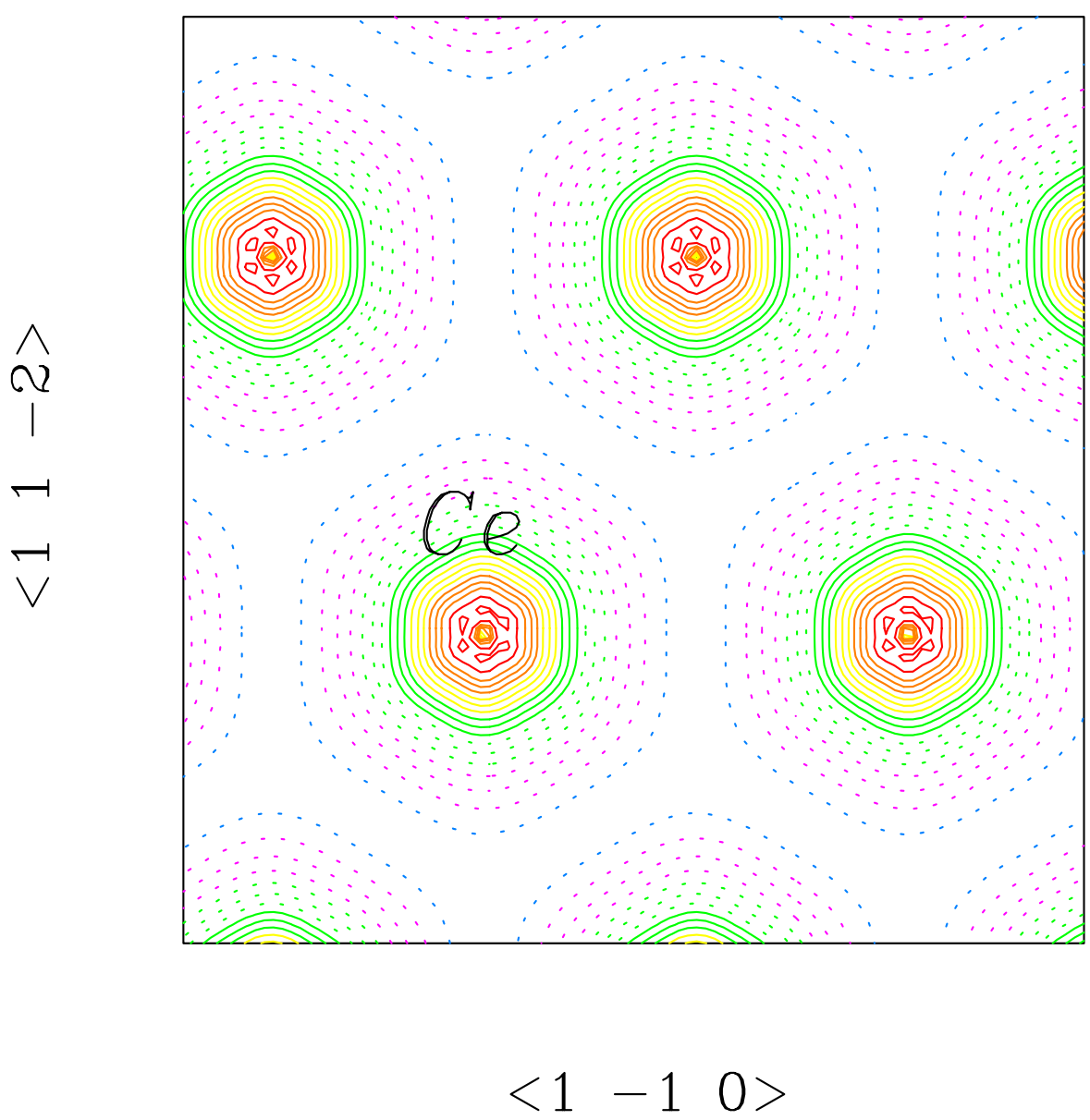


$m_{l}$ when orbital polarization is present. The overall effect on the spin moment, however, is very small since most of the relevant changes occur above the Fermi energy. These graphs do indicate a slightly larger difference in the spin moment due to the orbital polarization for the chalcogenide compounds and less effect on the pnictide compounds due to the higher Fermi level of the chalcogenide systems (CeS) resulting from the extra $p$ electron.

In Table 3.2 we present the calculated values of the zero-temperature cerium magnetic moments (spin moment, orbital moment, and total moment) using the FPLMTO electronic structure calculations. We list the values with and without orbital polarization correction taken into account. In all cases we have found the spin and orbital polarizations to be in opposite directions as expected. Note the importance of the orbital polarization correction in the calculations for this series of compounds. Without the orbital polarization the total moments obtained are near zero, indicating a non-magnetic state. The orbital polarization correction increases the orbital moment by a much larger amount, making a total non-zero magnetic moment for these systems. Notice, however, even with the orbital polarization taken into account there is an overall failure of this method to predict magnetic moments consistent with the experimental values. This method fails to obtain the saturated moments for the heavier cerium pnictides. Also, it fails to predict the moment suppression from CeSb to CeTe.

We next turn to examining the effect of the crystal field on the calculations and the results of our special study. We do this by suppressing the non-spherical parts of the potential inside of the muffin-tins and doing a warped muffin tin calculation. We then compare the results obtained (having no crystal field effects) to those from the full potential calculation which includes the crystal field. Notice, that this warped muffin tin

Table 3.1: Final self-consistent values of the $f$ state Racah parameters for the compounds studied in units of mRy.

\begin{tabular}{|c|c|c|c|c|c|c|c|}
\hline $\mathrm{CeS}$ & $\mathrm{CeSe}$ & $\mathrm{CeTe}$ & & $\mathrm{CeP}$ & $\mathrm{CeAs}$ & $\mathrm{CeSb}$ & $\mathrm{CeBi}$ \\
4.72 & 4.63 & 4.56 & & 4.47 & 4.44 & 4.43 & 4.39 \\
\hline
\end{tabular}


Figure 3.5: Cerium Phosphide f-projected density of states for spin up (shaded) and spin down (clear) electrons for the converged FPLMTO spin polarized potential without orbital polarization. The Fermi energy is marked on the graph and is given in Rydbergs.

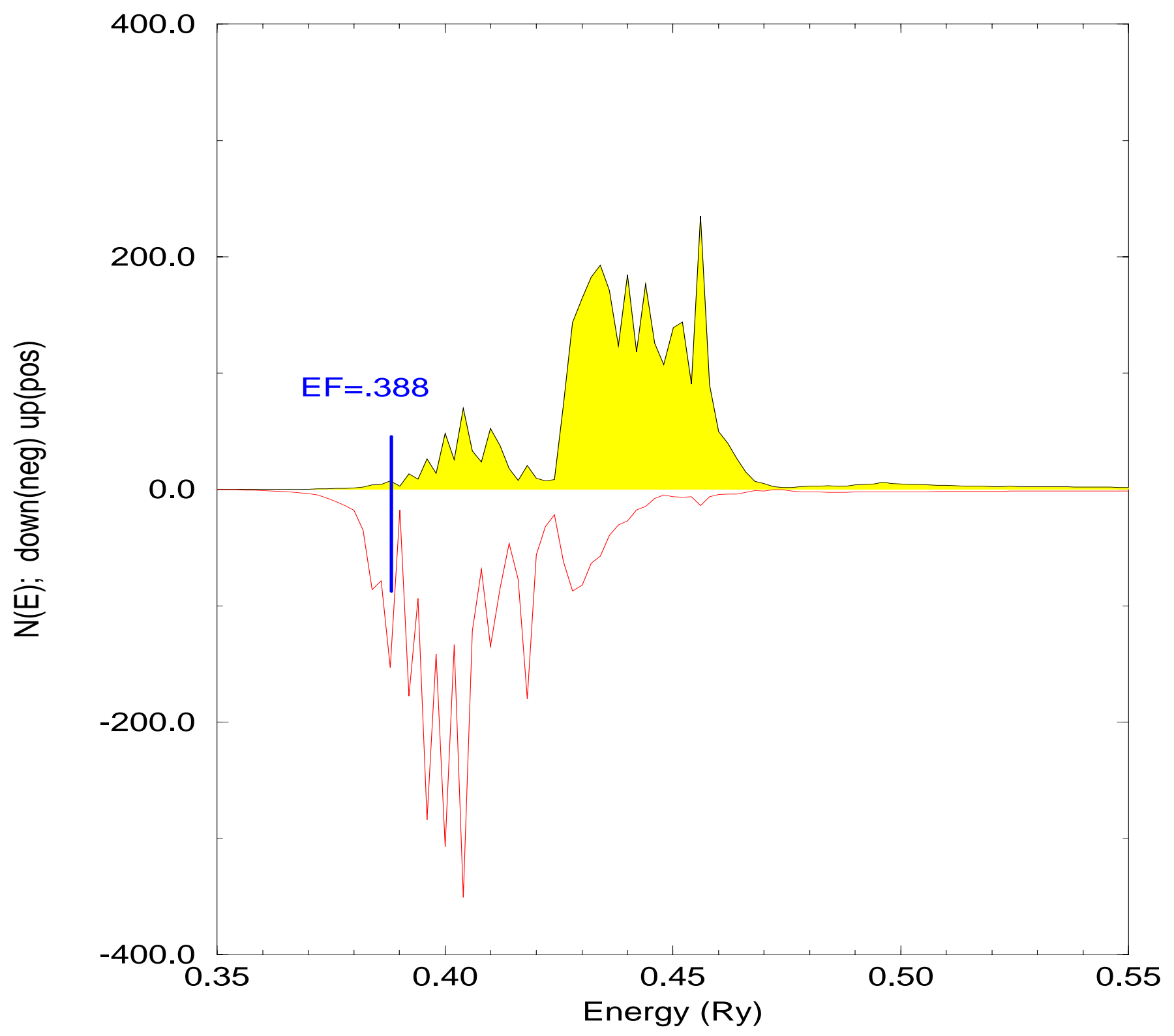


Figure 3.6: Cerium Phosphide $f$-projected density of states for spin up (shaded) and spin down (clear) electrons for the converged FPLMTO spin polarized potential with orbital polarization correction. The Fermi energy is marked on the graph and is given in Rydbergs.

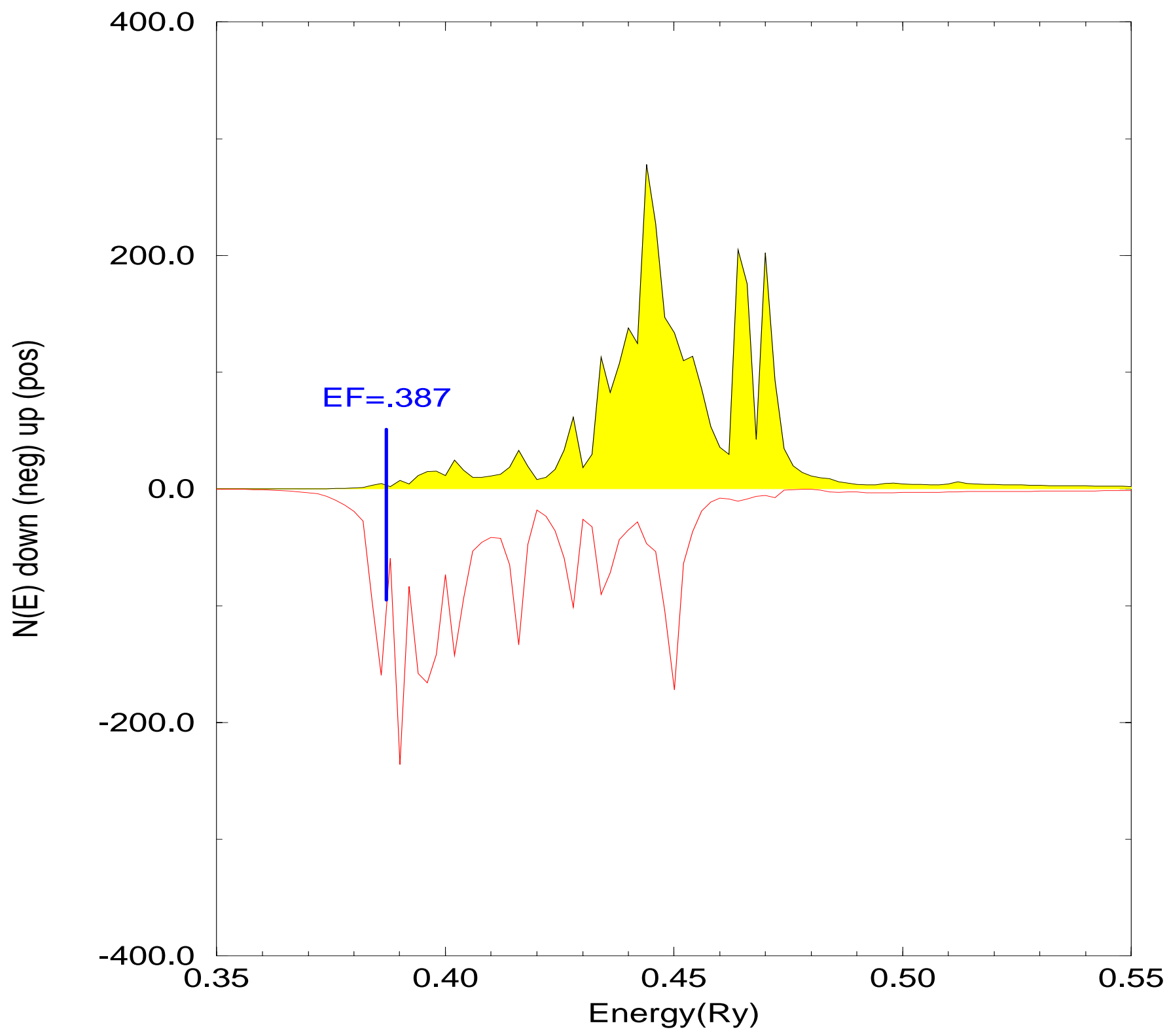


Figure 3.7: Cerium Sulfide $f$-projected density of states for spin up (shaded) and spin down (clear) electrons for the converged FPLMTO spin polarized potential without orbital polarization. The Fermi energy is marked on the graph and is given in Rydbergs.

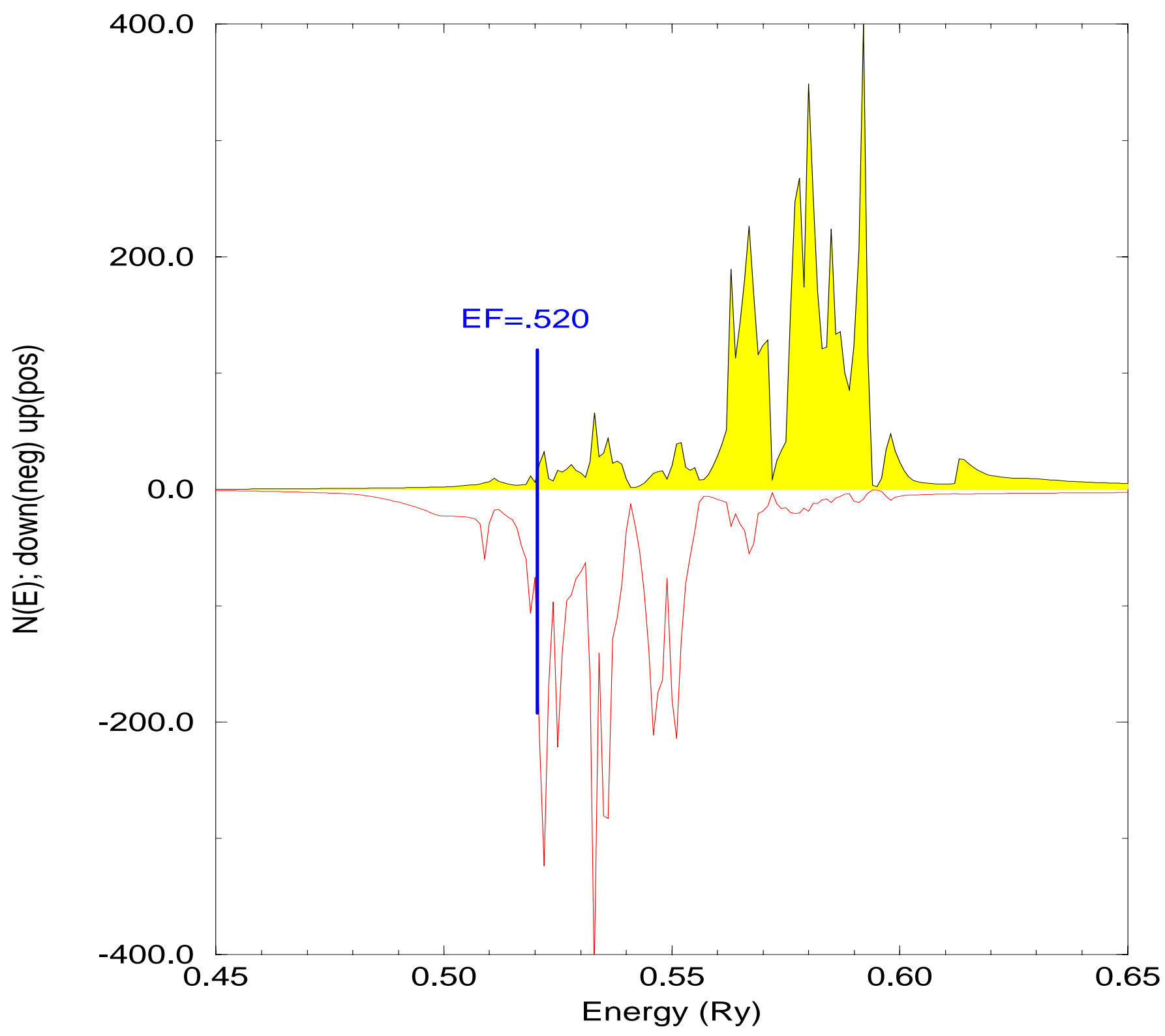


Figure 3.8: Cerium Sulfide $f$-projected density of states for spin up (shaded) and spin down (clear) electrons for the converged FPLMTO spin polarized potential with orbital polarization correction. The Fermi energy is marked on the graph and is given in Rydbergs.

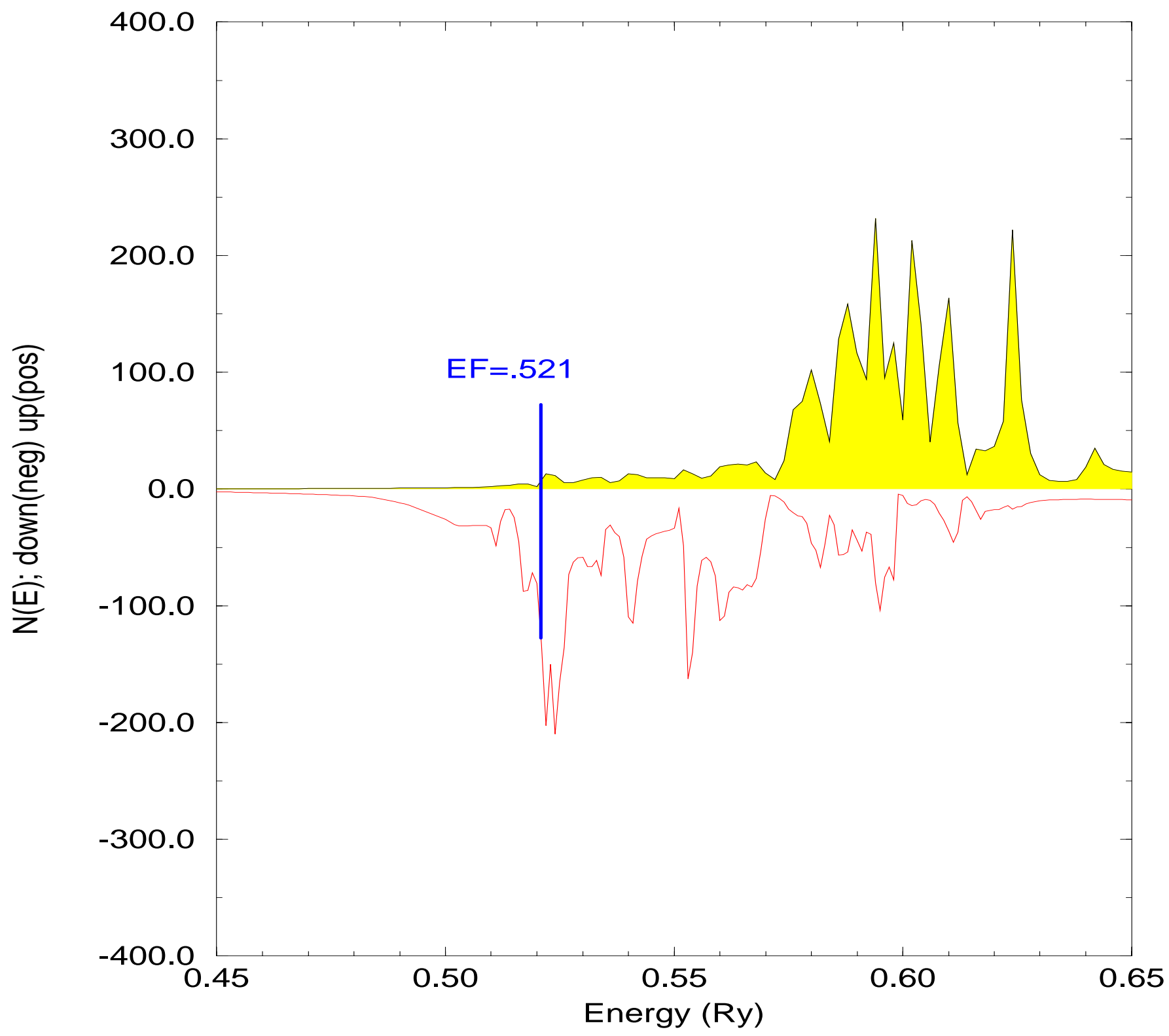


Table 3.2: Values of the calculated and experimental ${ }^{1-4}$ magnetic moments for the cerium chalcogenides and pnictides in units of $\mu_{B}$. Listed are the FPLMTO values for the spin moment $\mu_{S}$, the orbital moment $\mu_{L}$, and total moment $\mu$, for the spin polarized only calculation and for the calculation with spin polarization and orbital polarization correction.

FULL POTENTIAL

\begin{tabular}{|c|c|c|c|c|c|c|c|}
\hline & \multicolumn{2}{|c|}{$\mathrm{FP}+\mathrm{SP}$} & \multicolumn{2}{|c|}{$\mathrm{FP}+\mathrm{SP}+\mathrm{OP}$} & EXPT \\
& $\mu_{S}$ & $\mu_{L}$ & $\mu$ & $\mu_{S}$ & $\mu_{L}$ & $\mu$ & $\mu$ \\
\hline $\mathrm{CeS}$ & -1.00 & 0.91 & -0.09 & -1.24 & 1.99 & 0.75 & 0.57 \\
$\mathrm{CeSe}$ & -1.08 & 1.02 & -0.06 & -1.26 & 2.07 & 0.81 & 0.57 \\
$\mathrm{CeTe}$ & -1.15 & 1.28 & 0.07 & -1.31 & 2.29 & 0.98 & 0.30 \\
\hline $\mathrm{CeP}$ & -0.80 & 0.55 & -0.25 & -0.85 & 1.27 & 0.43 & 0.80 \\
$\mathrm{CeAs}$ & -0.84 & 0.64 & -0.20 & -0.85 & 1.42 & 0.57 & 0.80 \\
$\mathrm{CeSb}$ & -0.86 & 0.74 & -0.12 & -0.91 & 1.61 & 0.70 & 2.06 \\
$\mathrm{CeBi}$ & -0.86 & 0.74 & -0.12 & -0.95 & 1.69 & 0.74 & 2.10 \\
\hline
\end{tabular}


calculation also suppresses the crystal field on the non- $f$ basis states and is not directly analogous to the crystal field splitting of the $j=5 / 2$ manifold of the $f$ states. Table 3.3 shows the results of our special study. We have only included the results including orbital polarization noting that once again, the orbital polarization correction is required to obtain a non-paramagnetic moment.

We find that in all cases, inclusion of the harmonics within the muffin-tin reduces the calculated moments by a factor of 2 . The full potential produces results which are in better agreement with experiment for all compounds except the heavest cerium pnictides ( $\mathrm{CeSb}$ and $\mathrm{CeBi}$ ). In the cerium pnictides the moment reduction by the fullpotential is large and the total moments calculated all fall below the experimental values, this suggests that for these compounds the LDA calculation tends to overestimate the effect of the crystal field on the magnetic moments of the cerium pnictides because the hybridization dressing produces a reduced crystal field splitting in these compounds and the full-potential fails to take that reduction into account. ${ }^{15}$ However, even in the absence of a possibly "overestimated" crystal field effect, the moments for the heaviest pnictides remain well below the experimental values. Also, the moments for the chalcogenides, though reduced from the values calculated using the warped muffin-tin potential, are not reduced enough. If the inclusion of the non-spherical components of the potential overestimates the crystal field in this system of compounds, then we have to conclude that there must be some other feature of the cerium chalcogenide systems which is responsible for the reduction in the ordered moment of these compounds.

By comparing the unit cell energies of the converged potentials we find that the fullpotential calculations give a direction of anisotropy for the cerium pnictides of $\langle 001\rangle$ and $\langle 111\rangle$ for the cerium chalcogenides, in agreement with experiment. However, the warpedmuffin tin calculation only predicts the correct directions for some of these compounds. The symmetry of the $f$ orbitals is different for polarization along the $<001>$ axis compared to polarization along the $<111>$ axis. The full potential calculation allows for construction of $f$ orbitals in correspondence with these symmetries, whereas the warped muffin tin calculation neglects these differences. Our results indicate the importance of these differences and thus show the importance of using a full potential calculation 
Table 3.3: Values of the calculated magnetic moments for the cerium chalcogenides and pnictides in units of $\mu_{B}$. Listed are the warped muffin-tin LMTO values for the spin moment $\mu_{S}$, the orbital moment $\mu_{L}$, and total moment $\mu$ for the calculation with spin polarization and orbital polarization correction. For comparison we have also included the results for the total magnetic moment from the full potential calculation (FULL) and experimental results $(\mathrm{EXPT})^{1-4}$.

\begin{tabular}{|c|c|c|c|c|c|c|c|}
\hline & $\mathrm{CeS}$ & $\mathrm{CeSe}$ & $\mathrm{CeTe}$ & $\mathrm{CeP}$ & $\mathrm{CeAs}$ & $\mathrm{CeSb}$ & $\mathrm{CeBi}$ \\
$\mu_{S}$ & -1.32 & -1.32 & -1.31 & -0.83 & -0.89 & -0.99 & -1.01 \\
$\mu_{L}$ & 2.80 & 2.87 & 3.04 & 2.13 & 2.28 & 2.44 & 2.49 \\
$\mu$ & 1.48 & 1.55 & 1.73 & 1.30 & 1.39 & 1.45 & 1.48 \\
FULL & 0.75 & 0.81 & 0.98 & 0.43 & 0.57 & 0.70 & 0.74 \\
EXPT & 0.57 & 0.57 & 0.30 & 0.80 & 0.80 & 2.06 & 2.10 \\
\hline
\end{tabular}

Table 3.4: Energy differences obtained from the FPLMTO spin-polarized with orbital polarization correction between polarization along the $\langle 001\rangle$ direction and the $\langle 111\rangle$ direction. Values obtained from both the warped muffin-tin and full potential calculations are given. Positive values indicate polarization along the $\langle 001\rangle$ direction. Also listed are the experimental easy-axis directions. Values are given in $\mathrm{mRy}$.

\begin{tabular}{|c|c|c|c|} 
COMPOUND & $\begin{array}{c}E_{\langle 111\rangle}-E_{\langle 001\rangle} \\
\text { WARPED }\end{array}$ & $\begin{array}{c}E_{\langle 111\rangle}-E_{\langle 001\rangle} \\
\text { FULL POT }\end{array}$ & $\begin{array}{c}\text { EXPERIMENTAL } \\
\text { DIRECTION }\end{array}$ \\
\hline $\mathrm{CeP}$ & 0.4 & 0.8 & $\langle 001\rangle$ \\
$\mathrm{CeAs}$ & -0.2 & 1.0 & $\langle 001\rangle$ \\
$\mathrm{CeSb}$ & -0.4 & 0.7 & $\langle 001\rangle$ \\
$\mathrm{CeBi}$ & -0.4 & 1.9 & $\langle 001\rangle$ \\
\hline $\mathrm{CeS}$ & 0.2 & -5.1 & $\langle 111\rangle$ \\
$\mathrm{CeSe}$ & -0.8 & -1.0 & $\langle 111\rangle$ \\
$\mathrm{CeTe}$ & -0.7 & -1.6 & $\langle 111\rangle$ \\
\hline
\end{tabular}


when examining these compounds with LDA. The results of these calculations are given in Table 3.4 .

Overall, though, one observes that the LDA calculations are inconsistent and at best give a limited amount of information. The model of the cerium $f$ electron being placed into a valence band and allowed to hybridize freely provides an inadequate description of these systems. However, these calculations do show the importance of the orbital polarization correction and the use of a full-potential, indicating the significance of the crystal field, in calculating the magnetic properties. 


\subsection{MODEL HAMILTONIAN RESULTS}

To obtain the parameters in the model hamiltonian we use a FPLMTO electronic structure calculations with the $4 f$ states of cerium placed in the core and treated as atomic. The density of states for the cerium pnictides and chalcogenides without these $f$ states are shown in Figures 3.9 and 3.10 respectively. In the pnictide systems, the Fermi level appears in a pseudogap between the lower lying primarily $p$ band and the higher primarily $d$ band. However, the additional $p$ electron present in the chalcogenide systems raises the Fermi energy into the upper $d$ band, thus increasing the density of states at the Fermi level. For example, the density of states at the Fermi energy increases from $4.1 R y^{-1}$ in $\mathrm{CeSb}$ to $16.6 R y^{-1}$ in CeTe. This implies an increase in the hybridization and coulomb exchange interactions due to the large increase in band states available for interactions. This increase of involved band states shortens the effective wavelength of the two-ion interactions (oscillatory in nature), which dictate the magnetic coupling. The overall effect of this is to create a more antiferromagnetic environment in the chalcogenide system. Note also a distinction between the pnictides and chalcogenides is the appearance of a gap between the lower energy predominantly ligand $p$ states and the higher energy cerium $d$ states which appears in the chalcogenides, but not in the pnictides. Table 3.5 shows the calculated values for the Fermi energy, $E_{F}$ the core resonance cerium $4 f$ state energy, $E_{4 f}$ and the density of states at the Fermi energy, $N\left(E_{F}\right)$.

Recall that in the model hamiltonian the on-site coulomb repulsion, $U$, and the $f$-state energy $E_{f}$ are separated unlike the values from the pure LDA convergence. Therefore, supercell calculations are used to evaluate these parameters which enter into the model hamiltonian. A sample calculation of the supercell derived parameters is provided in Table 3.6, and the final results of these calculations are given in Table 3.7. These results are consistent with those obtained in previous calculations ${ }^{12}$.

We have examined the exchange interaction matrices for the first three nearest neighbor shells. Since the configurations examined are made of ferromagnetic planes with the moment perpendicular to those planes, the coupling between nearest neighbors in each plane is ferromagnetic and perpendicular to the bonding axis in which we have quan- 
Table 3.5: Summary of the FPLMTO results for the Fermi energy, $E_{F}$, the Ce $4 f$ core resonance energy $E_{4 f}$ and the total density of states $N\left(E_{F}\right)$ at $E_{F} . E_{F}$ and $E_{4 f}$ are given in Ry, the DOS are in states/Ry.

\begin{tabular}{|c|c|c|c|}
\hline COMPOUND & $E_{F}$ & $E_{4 f}$ & $N\left(E_{F}\right)$ \\
\hline $\mathrm{CeP}$ & .399 & .444 & 2.6 \\
$\mathrm{CeAs}$ & .390 & .410 & 2.8 \\
$\mathrm{CeSb}$ & .370 & .362 & 4.1 \\
$\mathrm{CeBi}$ & .394 & .353 & 7.5 \\
\hline $\mathrm{CeS}$ & .589 & .523 & 12.8 \\
$\mathrm{CeSe}$ & .555 & .472 & 14.0 \\
$\mathrm{CeTe}$ & .496 & .410 & 16.6 \\
\hline
\end{tabular}

Table 3.6: Sample calculation of the calculation of the energy difference, $E_{f}-E_{F}$ and the on-site coulomb repulsion, $U$, for CeS. Listed are the number of $f$ electrons placed at the central cerium site, $n_{f}$, the band calculated Fermi energy $E_{F}^{n}$, the core resonance band calculated $f$ state energy $E_{f}^{n}$, and the energy difference for each number of central site $f$ electrons, $\mathcal{E}^{n}$.

$$
\begin{aligned}
& \begin{array}{|c|r|r|r|}
\hline n_{f} & E_{F}^{n}(\mathrm{Ry}) & E_{f}^{n}(\mathrm{Ry}) & \mathcal{E}^{n}(\mathrm{Ry}) \\
0 & .584 & .092 & -.492 \\
1 & .585 & .523 & -.062 \\
2 & .556 & .938 & .382 \\
\hline
\end{array} \\
& E_{f}-E_{F}=\frac{1}{2}\left(\mathcal{E}^{0}+\mathcal{E}^{1}\right) * 13.6 \frac{\mathrm{eV}}{R y}=-3.77 \mathrm{eV} \\
& E_{f}-E_{F}+U=\frac{1}{2}\left(\mathcal{E}^{1}+\mathcal{E}^{2}\right) * 13.6 \frac{\mathrm{eV}}{R y}=2.18 \mathrm{eV} \\
& U=(2.18 \mathrm{eV})-(-3.77 \mathrm{eV})=5.95 \mathrm{eV}
\end{aligned}
$$


Figure 3.9: Cerium Chalcogenide density of non-f states computed with the $f$ electrons being placed into the "core" and not allowed to hybridize with the non-f states. Densities are given in states/Ry. Fermi energies are indicated with an arrow.

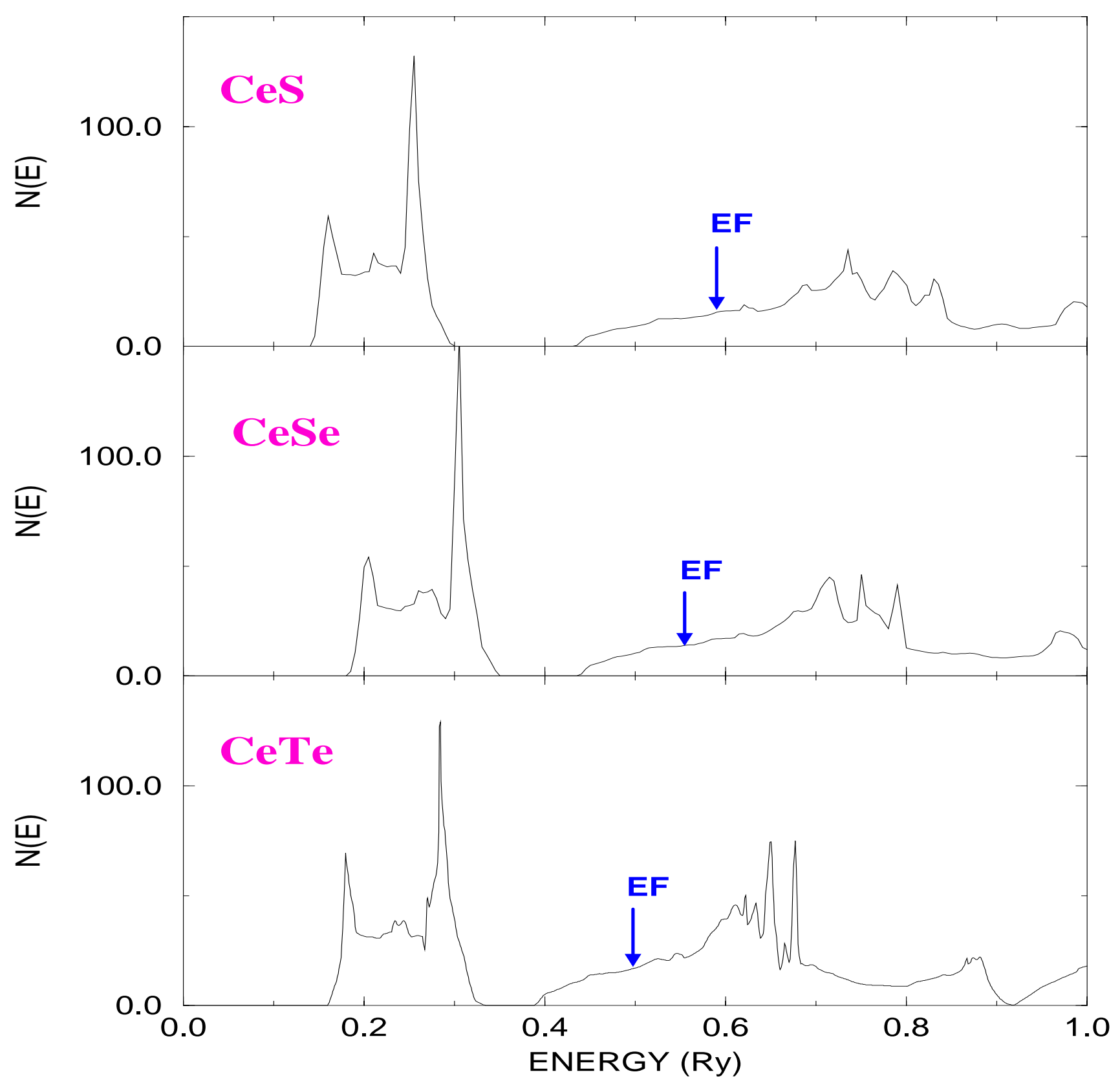


Figure 3.10: Cerium Pnictide density of non-f states computed with the f electrons being placed into the "core" and not allowed to hybridize with the non-f states. Densities are given in states/Ry. Fermi energies are indicated with an arrow.

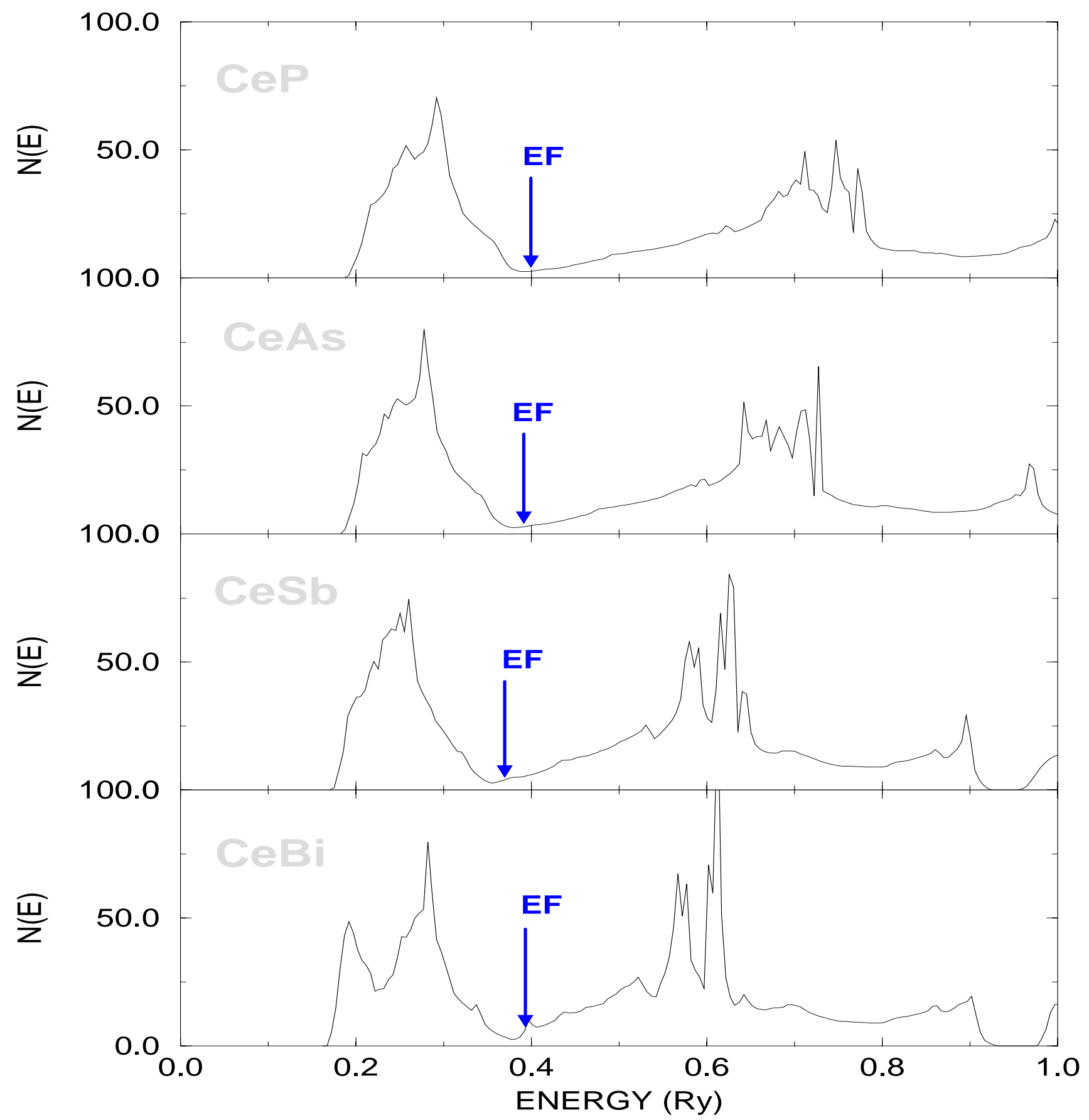


Table 3.7: Results from the supercell calculations for the energy difference $E_{f}-E_{F}$ and the on-site coulomb repulsion $U$ for the cerium compounds which appear in the model hamiltonian. All units are in $\mathrm{eV}$.

\begin{tabular}{|c|c|c|c|c|c|c|c|}
\hline & $\mathrm{CeS}$ & $\mathrm{CeSe}$ & $\mathrm{CeTe}$ & $\mathrm{CeP}$ & $\mathrm{CeAs}$ & $\mathrm{CeSb}$ & $\mathrm{CeBi}$ \\
$E_{f}-E_{F}$ & -3.8 & -3.7 & -3.0 & -2.2 & -2.1 & -2.0 & -2.0 \\
$\mathrm{U}$ & 6.0 & 6.1 & 6.3 & 5.9 & 6.0 & 6.0 & 6.0 \\
\hline
\end{tabular}

tized the two-ion interactions, thus it is the $m= \pm m^{\prime}= \pm \frac{1}{2}$ matrix elements which are characteristic of the systems. These are shown in Table 3.8 for the first three nearest neighbors of the light compounds ( $\mathrm{CeP}$ and $\mathrm{CeS}$ ) and the heavier ( $\mathrm{CeSb}$ and $\mathrm{CeTe}$ ). We list separately the contributions to the exchange interaction from pure hybridization $\left(V^{4}\right)$, the cross term $\left(V^{2} J\right)$, and the pure Coulomb exchange $\left(J^{2}\right)$. Positive values of these exchange parameters indicate ferromagnetic interactions, whereas negative values indicate antiferromagnetic interactions. Notice that for the smaller cerium compounds the pure hybridization exchange interaction is slightly larger than the Coulomb exchange mediated interactions. However, for the larger compounds the pure Coulomb exchange interaction dominates. This change indicates the sensitivity of the hybridization and Coulomb exchange on the degree of $4 f$ localization. Of equal importance is that while for the pnictide systems ( $\mathrm{CeSb}$ and $\mathrm{CeP}$ ), the first and second nearest neighbor interactions are all ferromagnetic; there is an interplay between ferromagnetic and antiferromagnetic interactions for the chalcogenide systems ( $\mathrm{CeS}$ and $\mathrm{CeTe}$ ), giving rise to the saturated moment for $\mathrm{CeSb}$ and the magnetic moment collapse from CeSb to CeTe (see Table 3.10). We have found that the second nearest neighbor interactions are slightly larger than those for the first nearest neighbor indicating a strong $\mathrm{Cef}$-Xp bonding. We have also found that the magnitudes of the exchange parameters are consistently larger for the cerium pnictides than in the corresponding chalcogenide systems, leading to larger ordering temperatures. We also list the $m= \pm m^{\prime}= \pm \frac{1}{2}$ elements of the total exchange interaction matrix in Table 3.9. Note that there is a change in sign between the total exchange interactions of the first and second nearest neighbors for the chalcogenides, 
but no such difference for the pnictides. Another difference is the larger off diagonal $m=-m^{\prime}= \pm \frac{1}{2}$ matrix elements in the second nearest neighbor interactions of the chalcogenides where the diagonal elements leading to a smaller ordered moment. The interactions of the third nearest neighbors, $R=\left(1 \frac{1}{2} \frac{1}{2}\right)$, are not listed. However, we note that these interactions are 15 to 30 times smaller than those of the first or second nearest neighbors.

Table 3.8: Listed below are characteristic matrix elements of the 6 X6 two-ion interaction matrix $\left(E_{m_{a} m_{a}^{\prime}}^{m_{b} m_{b}^{\prime}}\left(\mathbf{R}_{\mathbf{b}}-\mathbf{R}_{\mathbf{a}}\right)\right)$ of the $f$ electrons for a cross section of the cerium compounds. Values of the hybridization induced $\left(E_{V^{4}}\right)$, cross terms $\left(E_{V^{2} J}\right)$, and pure Coulomb exchange $\left(E_{J^{2}}\right)$ interactions are listed for the first, second, and third nearest cerium neighbors in units of $K$. The listed elements are the $m=m^{\prime}= \pm \frac{1}{2}$.

LIGHT COMPOUNDS

\begin{tabular}{|l||c|c|c||c|c|c|}
\hline \multicolumn{1}{|c||}{} & \multicolumn{3}{c|}{$\mathrm{CeP}$} & \multicolumn{4}{c|}{$\mathrm{CeS}$} \\
\hline & $E_{V^{4}}$ & $E_{V^{2} J}$ & $E_{J^{2}}$ & $E_{V^{4}}$ & $E_{V^{2} J}$ & $E_{J^{2}}$ \\
\hline $\mathrm{R}=\left(\begin{array}{lll}\frac{1}{2} & \frac{1}{2} & 0\end{array}\right)$ & 2.23 & 0.64 & 1.53 & 0.85 & -0.40 & 1.70 \\
\hline $\mathrm{R}=\left(\begin{array}{lll}1 & 0 & 0\end{array}\right)$ & 6.39 & 0.27 & 1.65 & -1.60 & 0.04 & -1.10 \\
\hline $\mathrm{R}=\left(\begin{array}{lll}1 & \frac{1}{2} & \frac{1}{2}\end{array}\right)$ & -0.08 & -0.02 & 0.16 & 0.38 & -0.16 & 0.13 \\
\hline
\end{tabular}

HEAVY COMPOUNDS

\begin{tabular}{|l||c|c|c||c|c|c|}
\hline \multicolumn{1}{|c||}{} & \multicolumn{3}{c|}{$\mathrm{CeSb}$} & \multicolumn{4}{c|}{$\mathrm{CeTe}$} \\
\hline & $E_{V^{4}}$ & $E_{V^{2} J}$ & $E_{J^{2}}$ & $E_{V^{4}}$ & $E_{V^{2} J}$ & $E_{J^{2}}$ \\
\hline $\mathrm{R}=\left(\begin{array}{lll}\frac{1}{2} & \frac{1}{2} & 0\end{array}\right)$ & 0.67 & 0.34 & 6.55 & 0.17 & -0.19 & 2.90 \\
\hline $\mathrm{R}=\left(\begin{array}{lll}1 & 0 & 0\end{array}\right)$ & 1.92 & 0.07 & 8.78 & -0.19 & 0.04 & -1.69 \\
\hline $\mathrm{R}=\left(\begin{array}{lll}1 & \frac{1}{2} & \frac{1}{2}\end{array}\right)$ & -0.02 & -0.03 & 0.40 & 0.04 & -0.06 & -0.01 \\
\hline
\end{tabular}

Figure 3.11 shows the calculated ordered moment for $\mathrm{CeSb}$ as a function of temper- 
Table 3.9: Matrix elements from the total two ion interaction $E_{m_{a} m_{a}^{\prime}}^{m_{b} m_{b}^{\prime}}\left(\mathbf{R}_{\mathbf{b}}-\mathbf{R}_{\mathbf{a}}\right)=E_{V^{4}}+$ $E_{V^{2} J}+E_{J^{2}}$ where $m= \pm \frac{1}{2}$ and $m^{\prime}= \pm \frac{1}{2}$ for the first and second nearest neighbors.

\begin{tabular}{|c|c|c|c|}
\hline CHALCOGENIDES & $\mathbf{R}=\left(\frac{1}{2} \frac{1}{2} 0\right)$ & $\mathbf{R}=$ & $(100)$ \\
\hline $\mathrm{COS}$ & $+1.9+1.5$ & -2.4 & -3.2 \\
\hline & $+1.5+1.9$ & -3.2 & -2.4 \\
\hline $\mathrm{CeSe}$ & $+2.3+1.9$ & -1.9 & -2.7 \\
\hline & $+1.9+2.3$ & -2.7 & -1.9 \\
\hline CeTe & $+2.9+2.4$ & -1.9 & -2.8 \\
\hline & $+2.4+2.9$ & -2.8 & -1.9 \\
\hline PNICTIDES & $\mathbf{R}=\left(\frac{1}{2} \frac{1}{2} 0\right)$ & $\mathbf{R}=$ & $(100)$ \\
\hline$C D$ & $+4.4+4.2$ & +8.3 & +7.2 \\
\hline & $+4.2+4.4$ & +7.2 & +8.3 \\
\hline $\mathrm{CeAs}$ & $+5.6+5.1$ & +9.8 & +8.1 \\
\hline & $+5.1+5.6$ & +8.1 & +9.8 \\
\hline $\mathrm{CeSh}$ & $+7.5 \quad+6.9$ & +11 & +8.9 \\
\hline & $+6.9+7.5$ & +8.9 & +11 . \\
\hline $\mathrm{CeBi}$ & $+25 . \quad+23$ & +37 . & +32 . \\
\hline & $+23 . \quad+25$ & +32 . & +37 . \\
\hline
\end{tabular}


ature both with and without the crystal field interactions. As the temperature slowly increases we notice very little effect on the ordered moment until the critical temperature is reached where there is a phase transition to the paramagnetic state.

Listed in Table 3.10 are the calculated zero-temperature ordered moments, $\mu_{0}$, and ordering temperatures, $T_{N}$, for the model hamiltonian method both with and without the crystal field interaction. It is clear that for the heavier systems ( $\mathrm{CeBi}, \mathrm{CeSb}, \mathrm{CeTe}$ ) the effect of the CF interaction on the magnetic moments is small and only slightly more pronounced on the ordering temperatures. This is due to the fact that for the more localized systems the CF interaction is smaller than the exchange interactions. For this reason, the previous calculations ${ }^{12}$ which neglected the CF interactions gave results in very good agreement with experiment. For the smaller more delocalized systems the CF interactions are much larger and hence dominate the magnetic behavior. The overall decrease of the magnetic moments in the presence of the $\mathrm{CF}$ interaction arises from the mixing of the off-diagonal angular momentum states $\mid \pm 5 / 2>$ with $\mp 3 / 2>$ states for the CF interaction with a $\Gamma_{7}$ ground state. Recall that the reduction of the ordered moment by the crystal field was also a conclusion the special study comparing the warped muffin tin calculated moments with those of the full potential from the pure LDA spin polarized method, thus both methods are consistent in this respect and in agreement with what one expects from the mixing of the states. Note that without the crystal field, the exchange alone predicts moments which are too high for all but the heaviest of these compounds, and in the case of the pnictides, they are completely saturated. Also, note that the moment from a pure crystal field with a $\Gamma_{7}$ ground state $\left(.71 \mu_{B}\right)$ is comparable to those of the lighter compounds but cannot account for the moments of the heavier compounds. Only by combining both the exchange interactions (including hybridization and Coulomb exchange) and the crystal field splitting do we obtain agreement with experiment for full range of cerium compounds. Overall, we find an excellent agreement with experiment for both the zero-temperature ordered moment and the ordered temperature (a more stringent test for the theory) using the $a b$ initio based model hamiltonian approach with all three pertinent interaction (hybridization, Coulomb exchange, and crystal field interactions) treated on an equal footing. 
Figure 3.11: Ordered moment versus temperature calculated from the mean field hamiltonian for CeSb for the lowest energy $\uparrow \uparrow \downarrow \downarrow$ configuration. Shown are both graphs calculated without and with the crystal field term (CF) taken into account. Indicated on the graph are the experimental results for the zero-temperature moment $2.06 \mu_{B}$ and the Néel temperature $17 \mathrm{~K}$.

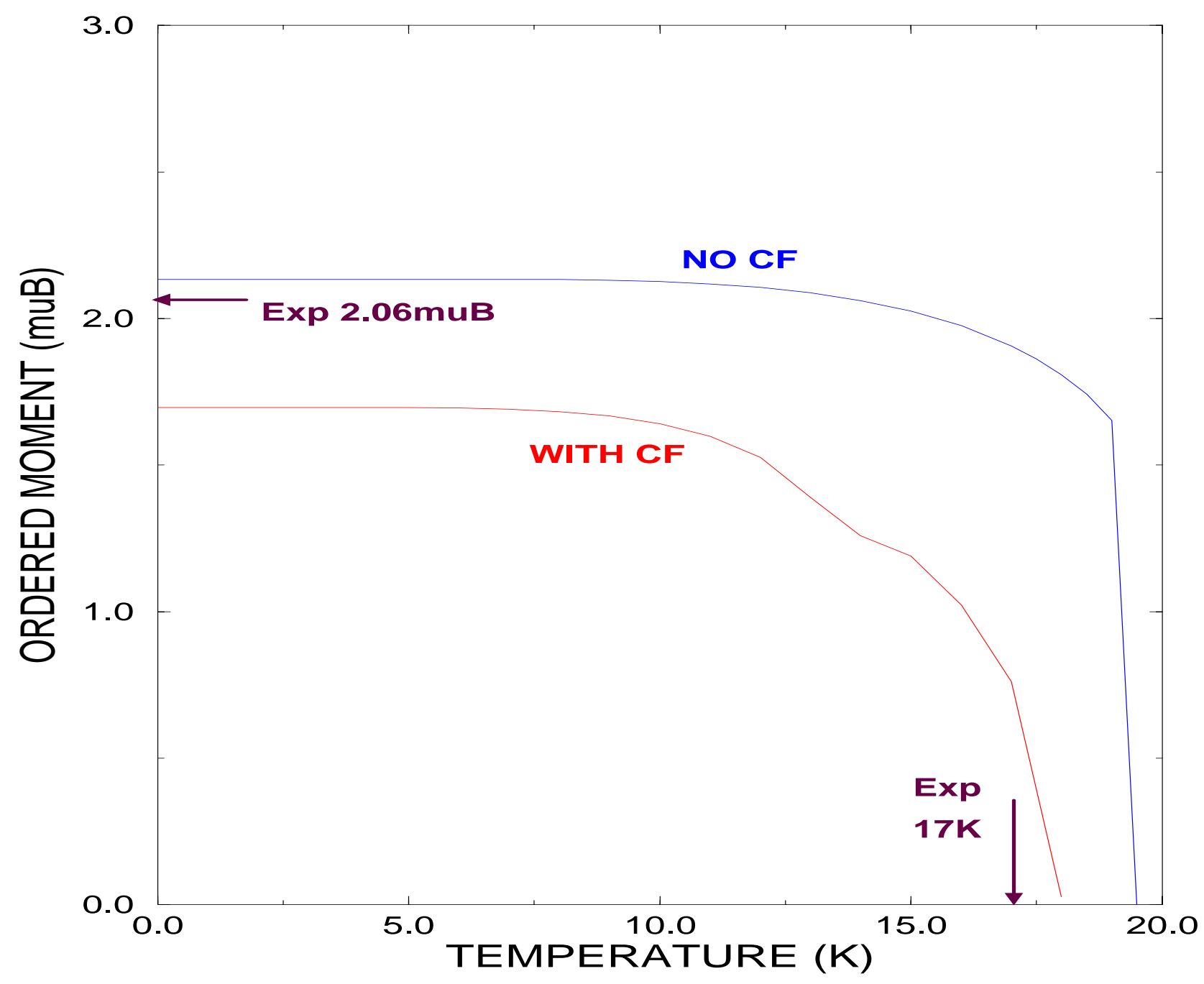


Table 3.10: Calculated (from the model hamiltonian) values of the zero temperature ordered moments $\left(\mu_{0}\right)$ and ordering temperatures, Néel temperature $\left(T_{N}\right)$ both without (no cf) and with the crystal field splitting term (cf), and the corresponding experimental values for the cerium compounds. The experimental values of the crystal field splitting $\left(\Delta_{C F}\right)$ are also listed. All moments are given in units of $\mu_{B}$, the temperatures and the crystal field splitting are given in units of Kelvin.

\begin{tabular}{|c|c|c|c|c|c|c|c|c|}
\hline & $\Delta_{C F}$ & \multicolumn{3}{|c|}{$\mu_{0}$} & \multicolumn{3}{|c|}{$T_{N}$} \\
\hline & & no cf & cf & exp & & no cf & cf & exp \\
\hline $\mathrm{CeS}$ & 140 & 1.80 & 0.73 & 0.57 & 1.0 & 11.0 & 8.4 \\
$\mathrm{CeSe}$ & 116 & 1.10 & 0.79 & 0.57 & 2.5 & 14.0 & 5.7 \\
$\mathrm{CeTe}$ & 32 & 0.60 & 0.46 & 0.30 & 8.0 & 5.0 & 2.2 \\
\hline $\mathrm{CeP}$ & 150 & 2.10 & 0.73 & 0.81 & 14 & 11 & 8 \\
$\mathrm{CeAs}$ & 137 & 2.10 & 0.74 & 0.85 & 16 & 13 & 8 \\
$\mathrm{CeSb}$ & 37 & 2.10 & 1.80 & 2.06 & 20 & 18 & 17 \\
$\mathrm{CeBi}$ & 8 & 2.10 & 2.10 & 2.10 & 40 & 40 & 26 \\
\hline
\end{tabular}


We now turn to the compounds for which this theory has been most successful, the cerium pnictide systems, and examine the finer details of this calculation.

To further understand the effect of the crystal field on the magnetic moments of these cerium pnictide compounds we vary the size of the crystal field term and see how the moment changes for each of the compounds. As a sample of this study we include Figure 3.12 which shows the calculated zero-temperature ordered moment versus the size of the crystal field interaction term for a ferromagnetic configuration of cerium phosphide. Though specific behaviors may vary for different compounds and configurations there seem to be three general regions characterized by the size of the crystal field term compared to those of the exchange interactions. First, there is a region of low $\mathrm{CF}$ interaction where the hybridization and Coulomb exchanges dominate the behavior of the system. For the cerium pnictides, we find saturated moments $\left(2.1 \mu_{B}\right)$ when a small crystal field term is added. Next, there is a sharp transition region where the moment decreases from saturation when the CF term included is on the order of the two-ion exchange interactions. This region gives vary large changes in ordered moment for small changes in the crystal field term. The third distinct region is for large values of the crystal field, we see a dominance of the $\mathrm{CF}$ in the system producing ordered moments which approach the pure crystal field moment of . $71 \mu_{B}$. To estimate the uncertainty of the magnetic moments calculated from this method we change the size of the crystal field term entering into the model hamiltonian by the amount of its experimental uncertainty, where applicable, and examine the effects on the zero-temperature ordered moments for all cerium compounds. We note that in some cases, experimental uncertainties are not presented, therefore we use the differences in the crystal field splittings published by different groups to estimate this uncertainty. Listed in Table 3.11 are the estimated uncertainties for the moments of these compounds.

As an even further test of this theory, we now examine the energetics between various antiferromagnetic configurations of these compounds. We examine the temperature dependence on the energies of various ordered configurations to determine the ground state properties of these systems. Figure 3.13 and 3.14 show the calculated temperature dependence of the ground state energy of the converged mean-field calculation 
Figure 3.12: Zero-temperature ordered moment versus the crystal field splitting (CF) term entering the model hamiltonian for cerium phosphide the ordered moment are given in $\mu_{B}$. Three regions are indicated on the graph, the exchange dominated, the transition region, and the crystal field dominated.

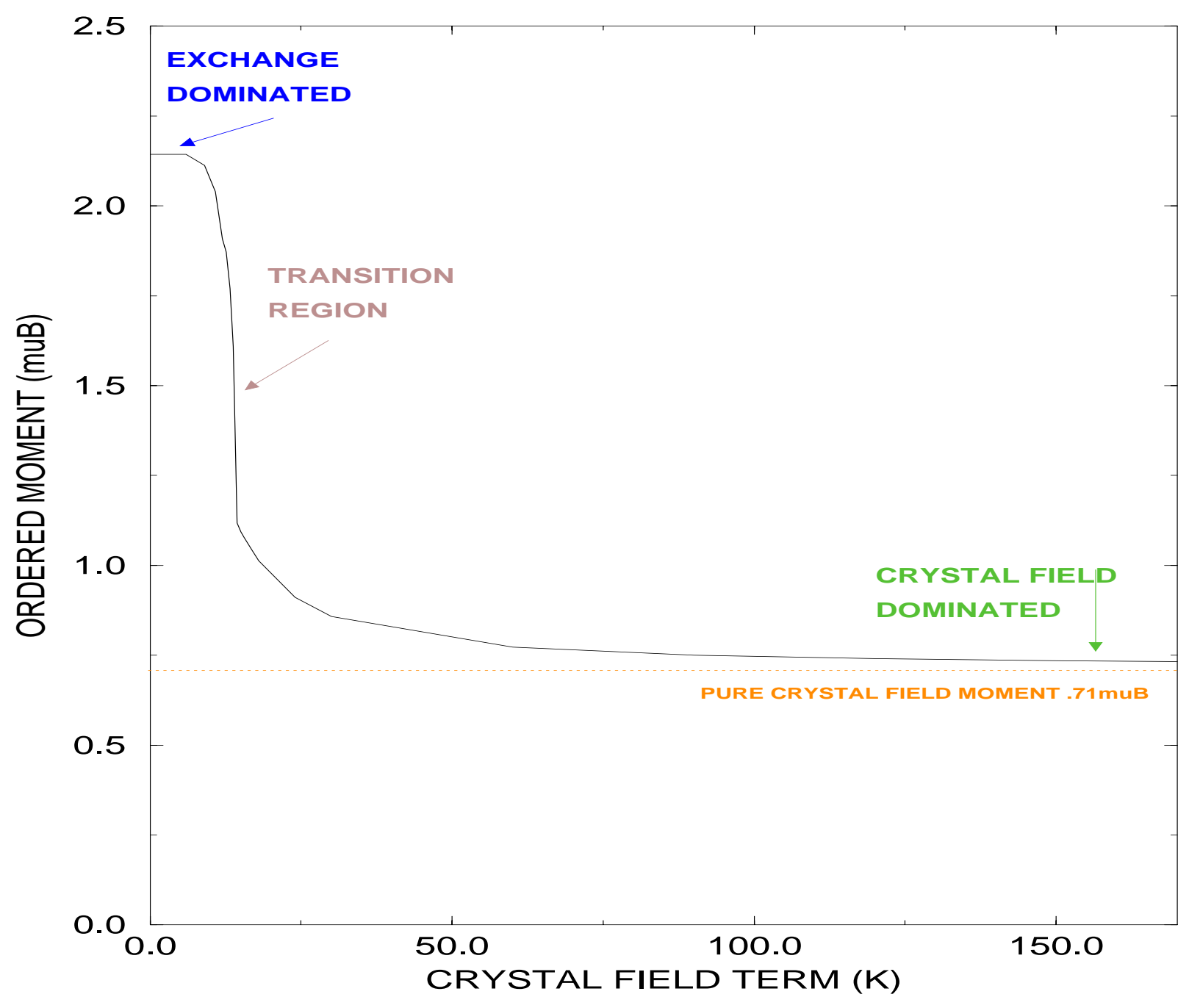


of the CeSb system for various antiferromagnetic configurations in which only the exchange interactions are used (Figure 3.13) and those where the crystal field correction is applied (Figure 3.14). Shown are the energies of the paramagnetic state, the type-I antiferromagnetic state $(\uparrow \downarrow)$, the type-IA antiferromagnetic state $(\uparrow \uparrow \downarrow \downarrow)$, and the AFP state $(\uparrow \downarrow 0)$ in which every third plane is nonmagnetic, at each temperature until the system no longer converges in a particular state; at which point the system converges in a paramagnetic state. From these energies we can obtain a calculated Néel temperature by examining where the energy of the ordered configuration becomes larger than the energy of the paramagnetic state. Both sets of calculations, with and without crystal field effects produce a ground state configuration of type-IA antiferromagnetic for $\mathrm{CeSb}$ for all temperatures up to the Néel temperature with a calculated ordering temperature of $19 \mathrm{~K}$ with no crystal field and $17 \mathrm{~K}$ when the crystal field is added to the hamiltonian. The experimental zero-temperature configuration of type-IA is predicted by both sets of results. However, the high-temperature ordered configuration of $\mathrm{CeSb}$ is an AFP configuration which according to both sets of calculations is not the ground state, however, note that when the crystal field is included in the hamiltonian, the energy of the AFP configuration is lowered with respect to the other configurations, indicating that the crystal field promotes the existence of a paramagnetic plane at higher temperatures. If we assume the experimental high temperature configuration and determine the ordering temperature we find that the Néel temperature is $17.5 \mathrm{~K}$ in excellent agreement with the experimental value of $17 \mathrm{~K}$.

Next, we examine the zero-temperature configurations for all cerium pnictide systems. The heavier pnictide systems ( $\mathrm{CeSb}$ and $\mathrm{CeBi}$ ) were found to have an antiferro-

Table 3.11: Estimated uncertainty in the calculated zero-temperature ordered moments for the cerium compounds based on an uncertainty in the experimental values of the crystal field splitting used in this calculation in units of $\mu_{B}$.

\begin{tabular}{|c|c|c|c|c|c|c|c|}
\hline & CeP & CeAs & CeSb & CeBi & CeS & CeSe & CeTe \\
$\sigma_{\mu}\left(\mu_{B}\right)$ & .03 & .05 & .10 & .02 & .02 & .02 & .10 \\
\hline
\end{tabular}


Figure 3.13: Energy (in Kelvin units) versus temperature (in Kelvin units) for the paramagnetic (PARA), AFP $(\uparrow \downarrow 0)$, type-I $(\uparrow \downarrow)$ and type-IA $(\uparrow \uparrow \downarrow \downarrow)$ configurations for $\mathrm{CeSb}$ calculated from the mean field hamiltonian with only the exchange interactions.

\section{CeSb (Energy of Vorious Configurotions) \\ NO CRYST AL VIKLD}

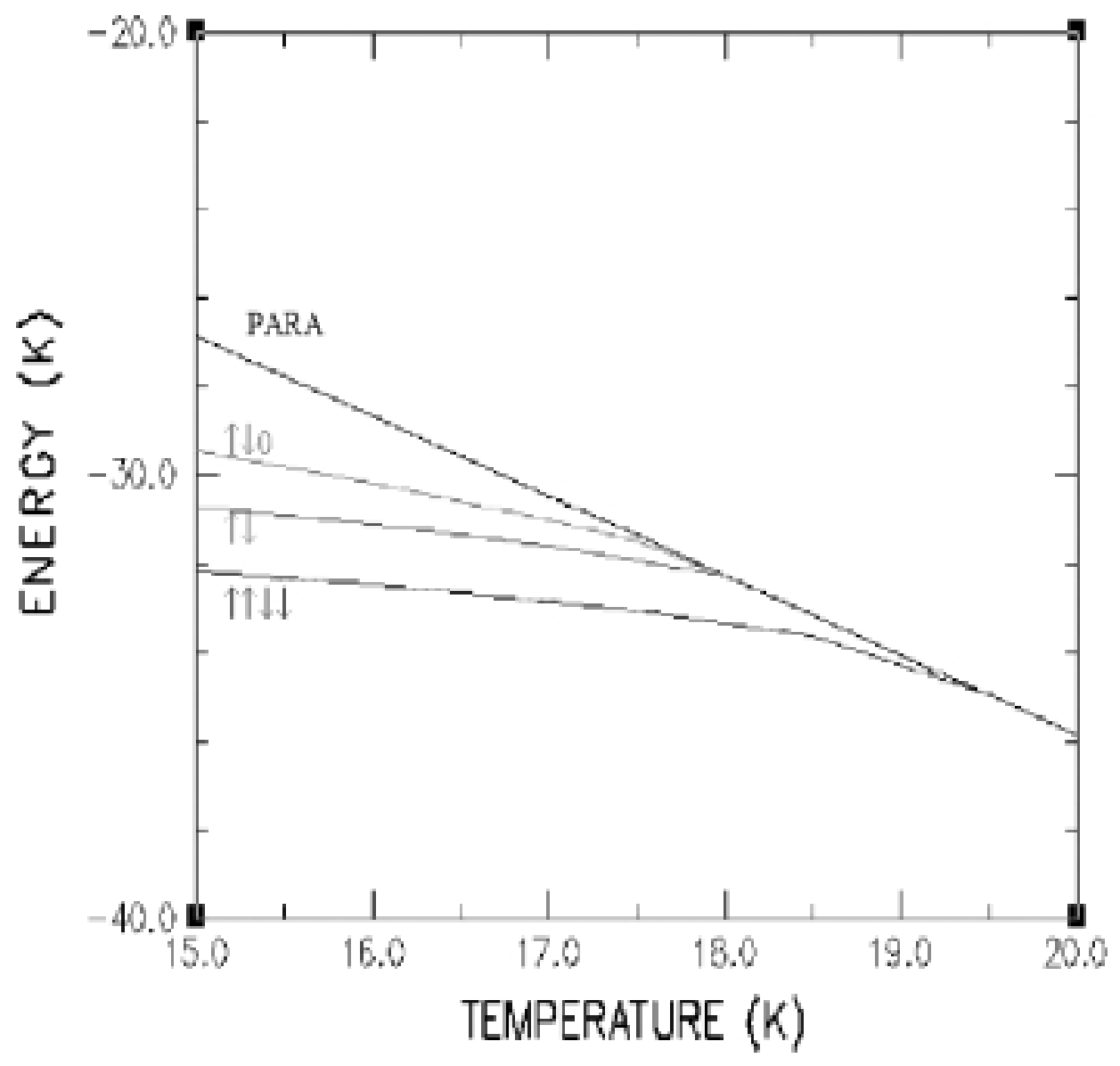


Figure 3.14: Energy (in Kelvin units) versus temperature (in Kelvin units) for the paramagnetic (PARA), AFP $(\uparrow \downarrow 0)$, type-I $(\uparrow \downarrow)$ and type-IA ( $\uparrow \uparrow \downarrow)$ configurations for $\mathrm{CeSb}$ calculated from the total mean field hamiltonian including all three pertinent interactions, (the hybridization, the Coulomb exchange and the crystal field).

\section{CeSb (Energy of Vorious Configurations)}

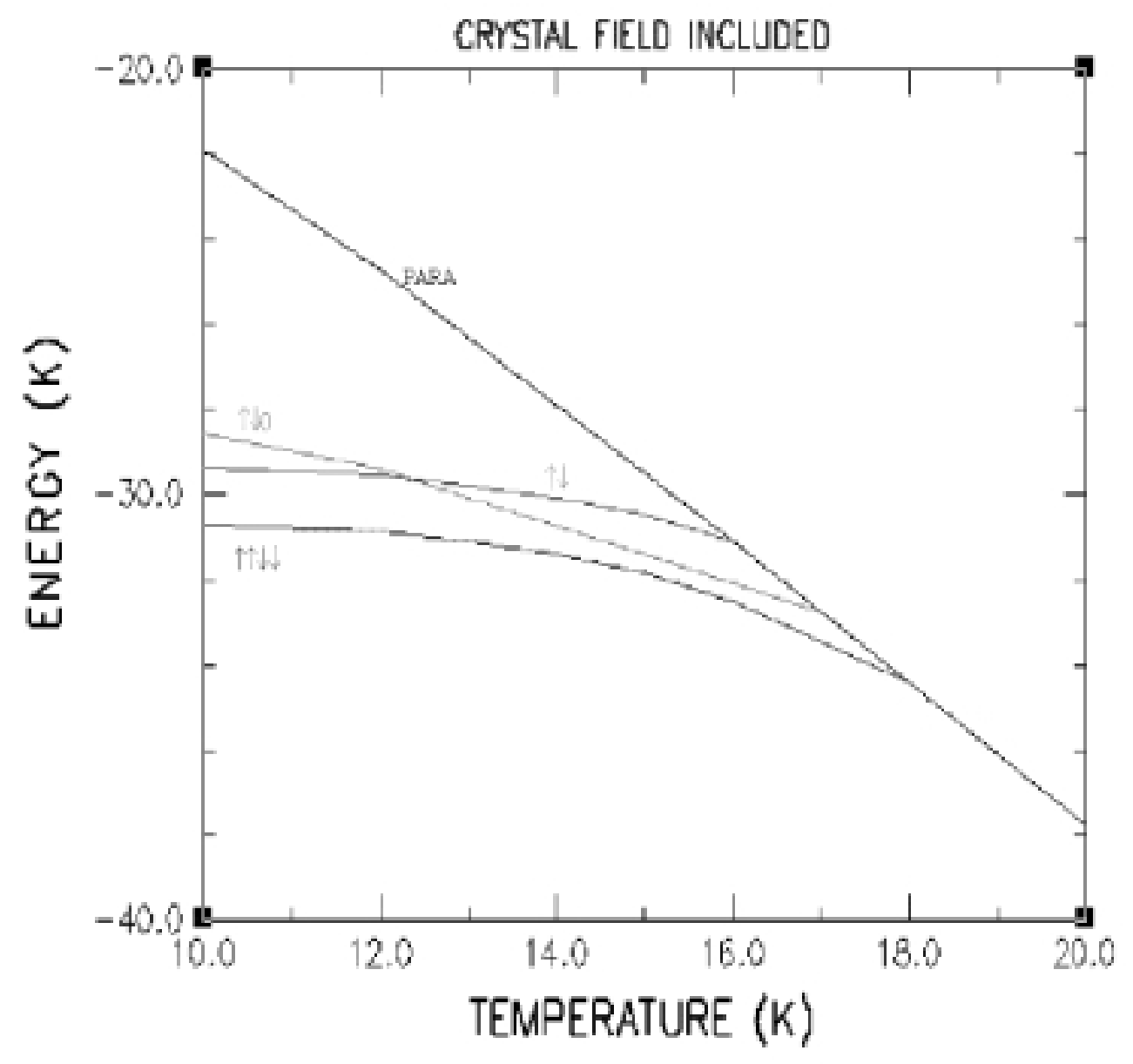


magnetic type-IA ground state configuration $(\uparrow \uparrow \downarrow \downarrow)$. The lighter cerium pnictides (CeP and CeAs) were found to favor a type-I antiferromagnetic $(\uparrow \downarrow)$ configuration over the type-IA configurations of the heavier cerium pnictides by $2 K$. These results both agree with experiment. ${ }^{1}$ To try and understand the reason for these results we examine the individual exchange interaction matrices for the hybridization and Coulomb exchange interactions for these compounds. Recall that for the inner plane ferromagnetism the

pertinent matrix element was the $m=m^{\prime}=\frac{1}{2}$ term. For coupling between the different planes, the larger $m$ values become important because the moments point more along the interatomic axis. Shown in Table 3.12 are the hybridization exchange and Coulomb exchange matrices for the first nearest neighbors of CeAs and CeSb. Notice the signs of the $m=m^{\prime}=5 / 2$ matrix elements (those found in the upper left corner) of each matrix. We see that for the pure hybridization exchange interactions that this first nearest neighbor plane interaction is antiferromagnetic and would tend to favor $(\uparrow \downarrow)$ configurations. The Coulomb exchange interaction matrix, on the other hand, has a ferromagnetic nearest neighbor plane interaction of the $m=m^{\prime}=5 / 2$ element and thus favors the $(\uparrow \uparrow \downarrow \downarrow)$. These results are independent of the crystal field interactions at least up to the experimental values used in this calculation. Table 3.13 gives the zero-temperature energies of the type-I and type-IA configurations for the cerium pnictides.

We find that even though we are limited somewhat in the study of the energetics at higher temperatures, we still obtain the correct zero-temperature configurations of the cerium pnictide systems which is a huge success of this model for understanding the mechanisms responsible for the unusual magnetic properties in this series of compounds.

\subsection{SUMMARY}

We have applied two different $a b$ initio based methods to study the dramatic change of magnetic properties across the cerium compounds which are strongly correlated and offer the opportunity to chemically tailor the different types of interactions (hybridization induced, Coulomb exchange, and crystal field interactions), important to the magnetic behavior, upon fine tuning the lattice constant. While the fully ab initio FPLMTO spin 
Table 3.12: First nearest neighbor exchange interaction matrices for CeAs and $\mathrm{CeSb}$ in units of K, shown separately for the hybridization (HYB) and pure Coulomb exchange (COUL) interactions.

CeAs HYB

$$
\left(\begin{array}{rrrrrr}
-.617 & -.353 & -.186 & -.186 & -.353 & -.617 \\
-.353 & -.017 & .611 & .611 & -.017 & -.353 \\
-.186 & .611 & 2.138 & 2.138 & .611 & -.186 \\
-.186 & .611 & 2.138 & 2.138 & .611 & -.186 \\
-.353 & -.017 & .611 & .611 & -.017 & -.353 \\
-.617 & -.353 & -.186 & -.186 & -.353 & -.617
\end{array}\right)
$$

CeSb HYB
CeAs COUL

$$
\left(\begin{array}{rrrrrr}
.283 & .095 & .024 & -.023 & -.046 & -.099 \\
.095 & .687 & .757 & .682 & .490 & -.046 \\
.024 & .757 & 2.876 & 2.676 & .682 & -.023 \\
-.023 & .682 & 2.676 & 2.876 & .757 & .024 \\
-.046 & .490 & .682 & .757 & .687 & .095 \\
-.099 & -.046 & -.023 & .024 & .095 & .283
\end{array}\right)
$$

\section{$\mathrm{CeSb}$ COUL}

$$
\left(\begin{array}{rrrrrr}
.518 & .181 & .079 & -.024 & -.030 & -.121 \\
.181 & 1.227 & 1.712 & 1.570 & 1.008 & -.030 \\
.079 & 1.712 & 6.550 & 6.230 & 1.570 & -.024 \\
-.024 & 1.570 & 6.230 & 6.550 & 1.712 & .079 \\
-.030 & 1.008 & 1.570 & 1.712 & 1.227 & .181 \\
-.121 & -.030 & -.024 & .079 & .181 & .518
\end{array}\right)
$$

Table 3.13: Ground state energies of the type-I and type-IA configurations of the cerium pnictides at $\mathrm{T}=0$. All units are Kelvin.

\begin{tabular}{|c|c|c|c|} 
& $\uparrow \downarrow$ & $\uparrow \uparrow \downarrow \downarrow$ & EXPT \\
\hline $\mathrm{CeP}$ & -21.9 & -20.4 & $\uparrow \downarrow$ \\
$\mathrm{CeAs}$ & -25.1 & -23.8 & $\uparrow \downarrow$ \\
$\mathrm{CeSb}$ & -26.5 & -28.4 & $\uparrow \uparrow \downarrow \downarrow$ \\
$\mathrm{CeBi}$ & -44.4 & -46.9 & $\uparrow \uparrow \downarrow \downarrow$ \\
\hline
\end{tabular}


polarized electronic structure calculations give good results for the lighter chalcogenide systems it entirely fails to give, even qualitatively, the trend of the unusual magnetic behavior. On the other hand, the model hamiltonian approach which explicitly takes into account the interplay of the three pertinent interactions, gives results in excellent agreement with experiment for the zero temperature ordered moments and the ordering temperature for all compounds in this series, and also correctly obtains the moment collapse from $\mathrm{CeSb}$ to $\mathrm{CeTe}$. The remaining problem of determining a full $a b$ initio calculation of the suppressed crystal-field interactions in this class of systems poses a theoretical challenge for future work. 


\section{Chapter 3 References}

1. J. Rossat-Mignod, P. Burlet, S. Quezel, J. M. Effantin, D. Delacôte, H. Bartholin, O. Vogt, and D. Ravot, J. Magn. Magn. Mater. 31-34, 398 (1983).

2. B. R. Cooper, R. Siemann, D. Yang, P. Thayamballi, and A. Banerjea, in The Handbook of the Physics and Chemistry of the Actinides, edited by A. J. Freeman and G. H. Lander (North-Holland, Amsterdam, 1985), Vol. 2, Chap. 6, pp. 435500.

3. A. Dönni, A. Furrer, P. Fischer, and F. Hulliger, Physica B 186-188, 541 (1993).

4. F. Hulliger, B. Natterer, and H. R. Ott, J. Magn. Magn. Mater. 8, 87 (1978).

5. H. R. Ott, J. K. Kjems, and F. Hulliger, Phys. Rev Lett. 42, 1378 (1979).

6. J. Rossat-Mignod, J. M. Effantin, P. Burlet, T. Chattopadhyay, L. P. Regnault, H. Bartholin, C. Vettier, O. Vogt, D. Ravot, and J. C. Achart, J. Magn. Magn. Mater. 52, 111 (1985).

7. N. Môri, Y. Okayama, H. Takahashi, Y. Haga, and T. Suzuki, Physica B 186-188, 444 (1993).

8. R. J. Birgeneau, E. Bucher, J. P. Maita, L. Passell, and K. C. Turberfield, Phys. Rev. B 8, 5345 (1973).

9. H. L. Skriver, The LMTO Method (Springer, Berlin, 1984).

10. D. L. Price and B. R. Cooper, Phys. Rev. B 39, 4945 (1989).

11. O. Eriksson, M. S. S. Brooks, and B. Johansson, Phys. Rev. B 41, 7311 (1990).

12. Q. G. Sheng and B. R. Cooper, J. Appl. Phys. 69, 5472 (1991); Phys. Rev. B 50,965 (1994).

13. N. Kioussis, B. R. Cooper, and J. M. Wills, Phys. Rev. B 44, 10,003 (1991). 
14. K. R. Lea, M. J. M. Leask, and W. P. Wolf, J. Phys. Chem. Solids (Pergamon Press) 23, 1381 (1962).

15. S. P. Lim and B. R. Cooper, J. Appl. Phys. 73, 5433 (1993). 


\section{Chapter 4}

\section{RESULTS ON URANIUM COMPOUNDS}

In this chapter we present the results for the zero temperature ordered moment and ordering temperatures for the uranium compounds using the two ab initio based methods. We find that while the pure electronic structure calculations give better agreement with experiment for the smaller, more itinerant, uranium compounds the model hamiltonian method results are in better agreement for the larger, more localized compounds. 


\subsection{LDA RESULTS}

Much like the cerium compounds, we find that the spin polarized calculations of the uranium compounds reveal only a very small contribution to the overall moment from the anion atom sites. Figures 4.1 and 4.2 show the shift in spin- $f$ projected energy levels of the density of states due to the addition of orbital polarization. We do find that the spin moment in the uranium compounds are slightly more affected by orbital polarization than those of the cerium compounds because there are three $f$ electrons per uranium atom site. However, the change in the orbital moment is once again the dominant difference between the calculations done with only spin polarization (orbital polarization through spin-orbit coupling) and those which contain the explicit orbital polarization correction. Table 4.1 gives the final $f$ state Racah parameters for the orbital polarization correction.

In Table 4.2 we present the calculated values of the zero-temperature uranium magnetic moment in the ferromagnetic state. For the LDA calculations we list the values with and without orbital polarization correction taken into account.

The values of the moments with no orbital polarization correction are consistently lower than the experimental results by a factor of 2 for the heavier pnictides and chalcogenides, and by a factor of 3 for the lighter ones. With orbital polarization correction taken into account the values of the total moment increase and are in better agreement with experiment. These results indicate the importance of the effect of orbital polarization in the uranium compounds. Note, that in all cases the orbital moment is antiparallel (in accordance to Hund's third rule) to and larger than the spin moment.

Looking now only at those calculations which include orbital polarization we find

Table 4.1: Final self-consistent values of the $f$ state Racah parameters for the uranium compounds studied in units of mRy.

\begin{tabular}{|c|c|c|c|c|c|c|c|}
\hline US & USe & UTe & & UP & UAs & USb & UBi \\
3.74 & 4.01 & 3.88 & & 4.09 & 4.02 & 3.88 & 3.81 \\
\hline
\end{tabular}


Figure 4.1: Uranium Sulfide $f$-projected density of states for spin up (shaded) and spin down (clear) electrons for the converged FPLMTO spin polarized potential without orbital polarization. The Fermi energy is marked on the graph and is given in Rydbergs.

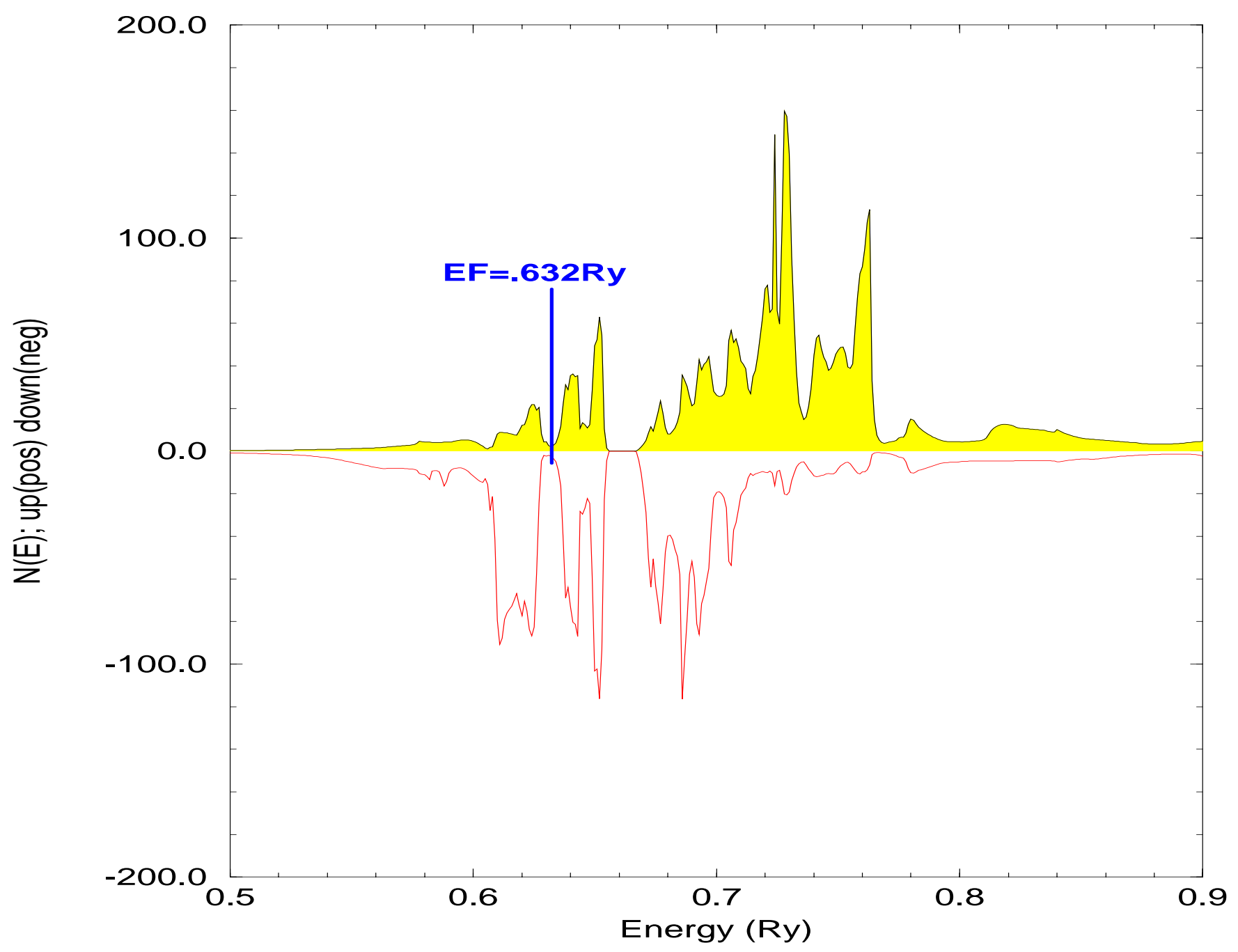


Figure 4.2: Uranium Sulfide f-projected density of states for spin up (shaded) and spin down (clear) electrons for the converged FPLMTO spin polarized potential with orbital polarization correction. The Fermi energy is marked on the graph and is given in Rydbergs.

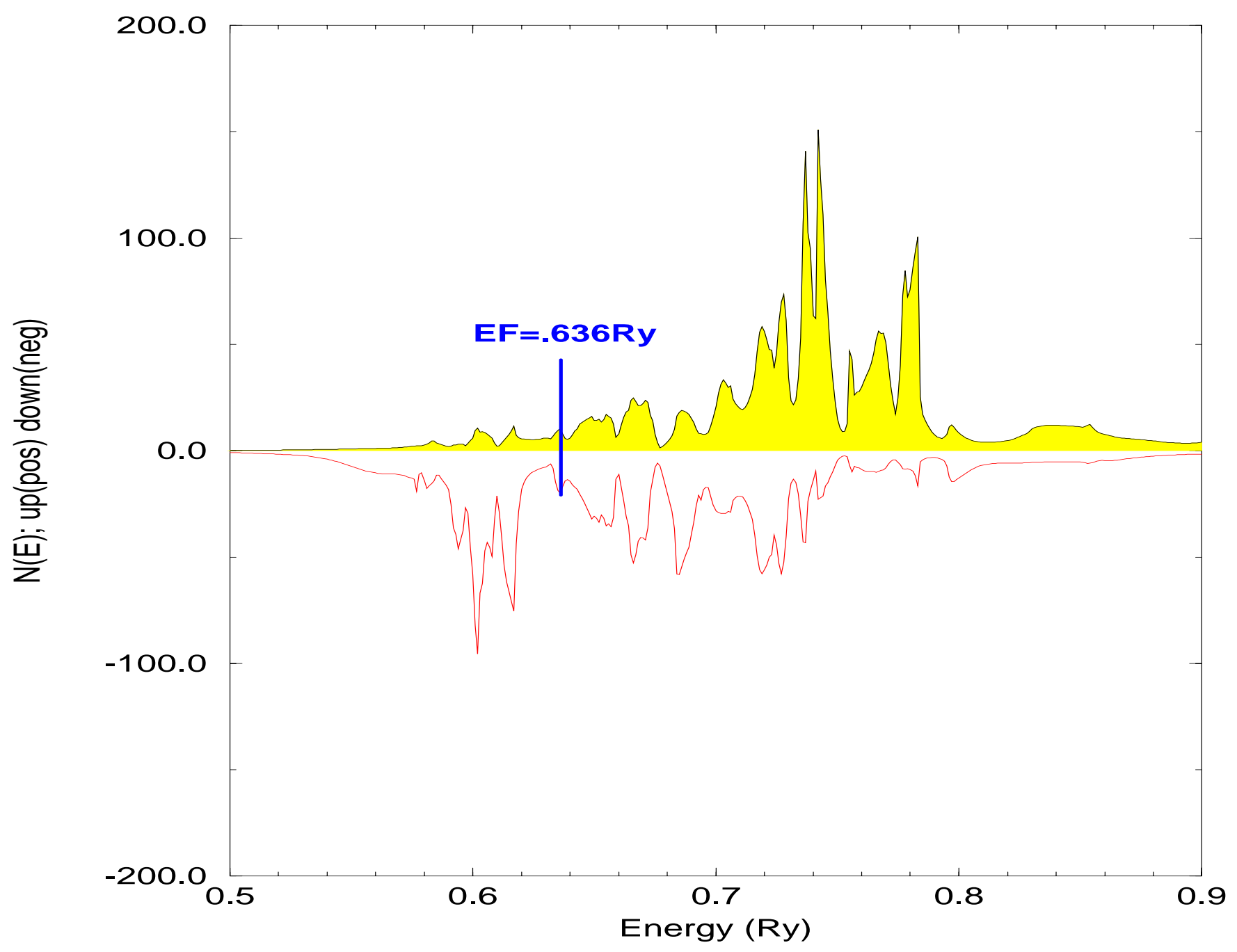


Table 4.2: Values of the calculated and experimental ${ }^{3,4,5}$ magnetic moments for the uranium chalcogenides and pnictides in units of $\mu_{B}$. Listed are the FPLMTO values for the spin moment $\mu_{S}$, the orbital moment $\mu_{L}$, and total moment $\mu$, for the spin polarized only calculation $(\mathrm{FP}+\mathrm{SP})$ and for the calculation with spin polarization and orbital polarization correction $(\mathrm{FP}+\mathrm{SP}+\mathrm{OP})$.

\begin{tabular}{|c|c|c|c|c|c|c|c|c|}
\hline & \multicolumn{3}{|c|}{$\mathrm{FP}+\mathrm{SP}$} & \multicolumn{2}{c|}{$\mathrm{FP}+\mathrm{SP}+\mathrm{OP}$} & EXPT \\
& $\mu_{S}$ & $\mu_{L}$ & $\mu$ & $\mu_{S}$ & $\mu_{L}$ & $\mu$ & $\mu$ \\
\hline $\mathrm{US}$ & -1.87 & 2.39 & 0.52 & -2.06 & 3.58 & 1.51 & 1.6 \\
$\mathrm{USe}$ & -2.01 & 2.68 & 0.67 & -2.27 & 3.89 & 1.62 & 2.0 \\
$\mathrm{UTe}$ & -2.35 & 3.23 & 0.88 & -2.60 & 4.26 & 1.66 & 2.2 \\
\hline $\mathrm{UP}$ & -2.18 & 2.66 & 0.48 & -2.43 & 3.85 & 1.43 & 1.8 \\
$\mathrm{UAs}$ & -2.32 & 3.00 & 0.68 & -2.44 & 4.12 & 1.68 & 2.2 \\
$\mathrm{USb}$ & -2.37 & 3.50 & 1.13 & -2.60 & 4.57 & 1.97 & 2.8 \\
$\mathrm{UBi}$ & -2.55 & 3.82 & 1.28 & -2.69 & 4.79 & 2.10 & 3.0 \\
\hline
\end{tabular}


that the results successfully predict the trend of increasing zero temperature magnetic moments with increasing lattice constants for both the chalcogenide and pnictide series. These calculations give better agreement with experiment for the lighter more itinerant chalcogenides (US) and pnictides (UP). On the other hand, they fail to give the nearly saturated moments for the heavier uranium compounds which exhibit a more localized nature of the $5 f$ states. Thus, for the more itinerant (lighter) systems where band theory is more appropriate, the refinement of including the orbital polarization corrections is relatively successful in providing agreement with experiment.

To determine the direction of magnetic anisotropy we have carried out total energy electronic structure calculations with the moment aligned in different directions. Table 4.3 shows the difference in total energy for the self consistent convergence of uranium compounds with the spin polarized in two different directions. For all uranium compounds the direction of anisotropy which gives the lowest total energy is the $\langle 111\rangle$ direction. This result agrees with experiment for the uranium chalcogenides, however fails to obtain the correct direction of anisotropy $(<001>)$ for the uranium pnictide compounds.

Table 4.3: Difference in energy of uranium systems converged with different directions of anisotropy. $E_{001}$ and $E_{111}$ are the total energies of the system spin and orbitally polarized along the $<001>$ and $<111>$ axes respectively. The units of the energy difference is given in $\mathrm{mRy}$

\begin{tabular}{|c|c|c|c|c|c|c|c|}
\hline & US & USe & UTe & UP & UAs & USb & UBi \\
$E_{001}-E_{111}(\mathrm{mRy})$ & 6.5 & 3.9 & 2.3 & 5.7 & 4.5 & 2.1 & 1.1 \\
\hline
\end{tabular}

We find that the purely $a b$ initio electronic structure calculations in which we have included orbital polarization correction are successful in calculating the magnetic properties of the lightest uranium compounds. However, this theory is very limited in its use and cannot account for the saturated moments of the larger more localized uranium compounds and also fails to predict the correct direction of anisotropy for the uranium pnictide systems. 


\subsection{MODEL HAMILTONIAN RESULTS}

When calculating the terms entering into the model hamiltonian for uranium compounds, we use an FPLMTO electronic structure calculation with the $f$ electrons placed in the valence but suppressing the hybridization. This allows us to examine directly the effects of the band- $f$ hybridization on the density of states for these compounds. The top two panels in Figure 4.3 shows the $f$ and non- $f$ density of states for uranium sulfide with the hybridization between the $f$ and non- $f$ band states suppressed, while the bottom panel is a plot of the total density of states for fully hybridized bands. One immediately can notice that fully hybridized density of states is not a simple addition of the unhybridized $f$ and non- $f$ density of states. This indicates the importance of the band- $f$ hybridization and the uranium $f$ state self banding which would be neglected if we were to adopt a "core" point of view for these compounds.

We now recount the choice of tail parameter $\kappa$ for the calculation of the hybridization from the band point of view. Table 4.4 gives the self-consistent results for various energy values calculated in the FPLMTO electronic structure of the uranium compounds. Due to the broadening of the extended uranium $f$ states, the band resonance $f$ state energies are all well above the Fermi energy for these compounds, and thus this energy is not a true representation of the filled $f$ states which hybridize. Therefore, we calculate the center of the occupied $f$ band after the potential has been converged, then set $\kappa^{2}$ equal to this energy to determine the hybridization. For all cases, we see that the energy of the occupied $f$ band is slightly below the Fermi energy, as one would expect. Also shown in the table is the self consistent interstitial kinetic energy. Notice that it is well below the level of the $f$ states and the Fermi energy. Primarily, the interstitial contains $p$ character electrons from the anions and we see very little contribution to this energy from the $f$ states. This indicates that though the uranium $f$ states are more extended than those of the cerium compounds, they still reside mainly inside of the muffin-tin sphere.

The results of the self-consistent supercell calculations of the difference in energy between the $f$ states and the Fermi energy $E_{f}-E_{F}$ and the on-site Coulomb repulsion $U$ are given in Table 4.5. Note that the values for the on-site Coulomb repulsion are 
Figure 4.3: Uranium Sulfide density of states. The top panel shows the density of unhybridized $f$ states and the density of the unhybridized non- $f$ states is shown in the middle panel. The bottom panel shows the fully hybridized total density of states.

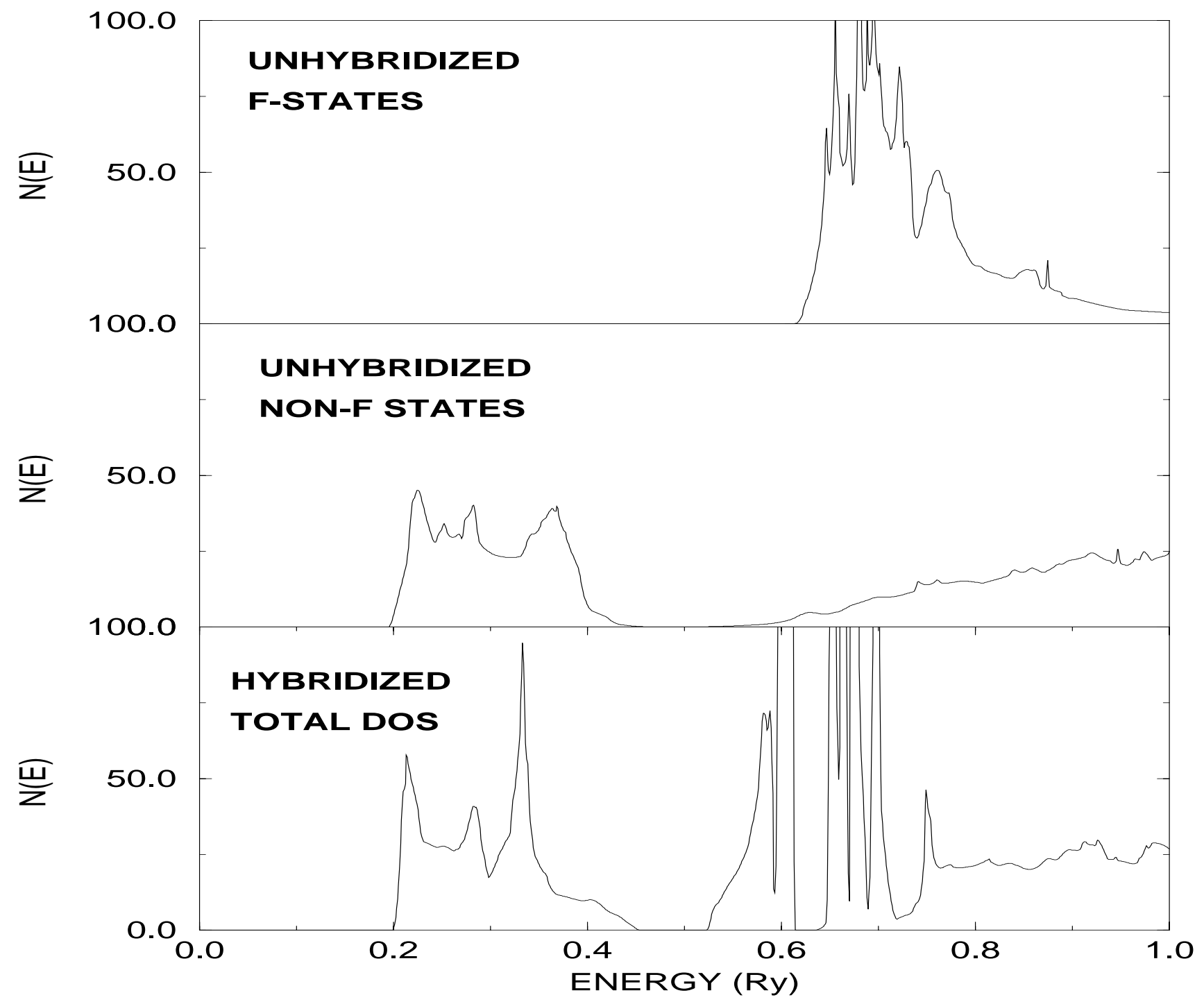


consistently larger for the pnictide systems than in the chalcogenide systems. Also, the addition of the $p$ electron in the chalcogenide systems raises the Fermi level so that there is more separation from the $f$-state energy level.

We now examine the interactions between uranium sites. By looking at the difference between the separate atomic charge densities and the final converged charge density of the crystalline structure, we can see where the charge has moved due to the crystal potential. Figures 4.4 4.5, and 4.6 show the bonding charge densities for US, UTe and UP respectively.

$$
\rho_{\text {bond }}=\delta \rho(\mathbf{r})=\rho_{\text {crystal }}(\mathbf{r})-\sum_{\alpha} \rho_{\alpha}\left(\mathbf{r}-\mathbf{R}_{\alpha}\right)
$$

In these plots the solid contours are charge accumulation and the dashed contours indicate regions of charge depletion in moving from the atomic to the crystal potential. In all the compounds shown, we can see a strong anion-anion bonding, most prevalent in UTe, where the atomic size of the tellurium is larger. The uranium atoms primarily bond to the nearby ligand atoms, once again more pronounced in UTe where there is less banding of the $f$ electrons. These results indicate a strong $f-p$ bond between the uranium atom and the respective anion. This suggests that the interactions between uranium sites, are mediated through the ligand atoms in the system.

We have calculated the $10 \times 10$ exchange interaction matrix pertinent to the change of magnetic behavior in this class of compounds. Characteristic values of the exchange interaction matrix elements $\left(m=m^{\prime}=\frac{5}{2}\right)$ of the $f^{3}$ multiplets for the first three nearestneighbor shells of US are listed in Table 4.6. The exchange interactions resulting from the hybridization of $f$ states with non- $f$ states are dominant due to the more extended spatial extent of the $5 f$ wavefunctions. Note, that the second nearest-neighbor exchange interaction is twice as large as the first nearest-neighbor exchange interaction. This is consistent with strong $f$ - $p$ hybridization seen in the bonding.

Listed in Table 4.7 are the calculated ordering temperatures $\left(T_{\text {ord }}\right)$ and ordered moments $\left(\mu_{0}\right)$ from the model hamiltonian method as well as the experimental values. The synthesis of phenomenology and electronic structure method correctly gives the trend of decreasing Curie temperatures with increasing lattice constant for the chalcogenides 
Table 4.4: Listed are the self-consistent energies from the FPLMTO electronic structure calculations for the uranium compounds. Shown are the interstitial kinetic energy $K E_{\text {int }}$, the center of the occupied $f$ valence band $E_{c o o f b}$, the Fermi energy $E_{F}$ and the resonance $f$ state energy $E_{f r e s}$. All energies are given in Rydbergs.

\begin{tabular}{|c|c|c|c|c|c|c|c|c|}
\hline & US & USe & UTe & & UP & UAs & USb & UBi \\
\hline$K E_{\text {int }}$ & .384 & .341 & .290 & & .387 & .359 & .302 & .282 \\
$E_{\text {coofb }}$ & .598 & .547 & .470 & .529 & .500 & .428 & .409 \\
$E_{F}$ & .604 & .552 & .474 & .538 & .507 & .433 & .412 \\
$E_{\text {fres }}$ & .651 & .596 & .518 & .583 & .549 & .473 & .451 \\
\hline
\end{tabular}

Table 4.5: Results of the self-consistent supercell calculations of the parameters entering into the two-ion interaction. Listed are the energy difference $E_{f}-E_{F}$, and the on-site Coulomb repulsion energy $U$. All units given are in $\mathrm{eV}$.

\begin{tabular}{|c|c|c|c|c|c|c|c|}
\hline & US & USe & UTe & UP & UAs & USb & UBi \\
$E_{f}-E_{F}(\mathrm{eV})$ & -1.58 & -2.20 & -2.07 & -0.55 & -0.99 & -1.25 & -1.58 \\
$U(\mathrm{eV})$ & 3.36 & 3.72 & 3.52 & 4.76 & 4.64 & 4.35 & 4.05 \\
\hline
\end{tabular}

Table 4.6: Characteristic matrix elements of the $10 \mathrm{X} 10$ two-ion interaction matrix $\left(E_{m_{a} m_{a}^{\prime}}^{m_{b} m_{b}^{\prime}}\left(\mathbf{R}_{\mathbf{b}}-\mathbf{R}_{\mathbf{a}}\right)\right)$ of the $f^{3}$ multiplet. Values of the hybridization induced $\left(E_{V^{4}}\right)$, cross terms $\left(E_{V^{2} J}\right)$, and pure Coulomb exchange $\left(E_{J^{2}}\right)$ interactions are listed for the $m=m^{\prime}=\frac{5}{2}$ elements for each of the first, second, and third nearest uranium neighbors in units of $\mathrm{K}$.

\begin{tabular}{|l|c|c|c|}
\hline & $E_{V^{4}}$ & $E_{V^{2} J}$ & $E_{J^{2}}$ \\
\hline $\mathrm{R}=\left(\begin{array}{lll}\frac{1}{2} & \frac{1}{2} & 0\end{array}\right)$ & 253 & 14.4 & 2.0 \\
\hline $\mathrm{R}=\left(\begin{array}{lll}1 & 0 & 0\end{array}\right)$ & 490 & 12.9 & 1.0 \\
\hline $\mathrm{R}=\left(\begin{array}{lll}1 & \frac{1}{2} & \frac{1}{2}\end{array}\right)$ & 29 & 4.2 & 0.1 \\
\hline
\end{tabular}


Figure 4.4: Uranium Sulfide bonding charge density, $\rho_{\text {bond }}$. The uranium atom sites are in the center and on the corners, the sulfur sites are on the sides.

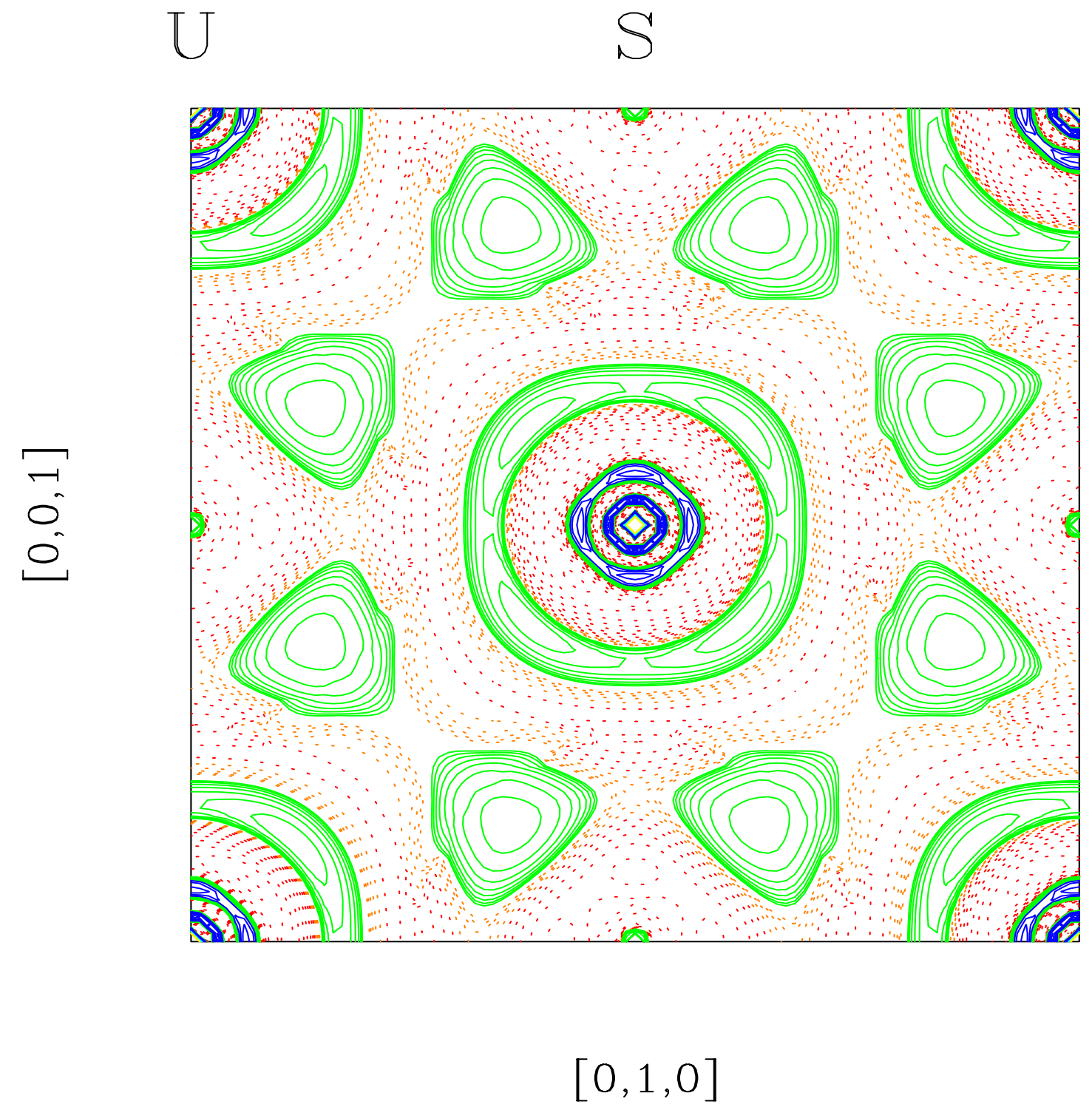


Figure 4.5: Uranium Telluride bonding charge density, $\rho_{\text {bond }}$. The uranium atom sites are in the center and on the corners, the tellurium sites are on the sides.

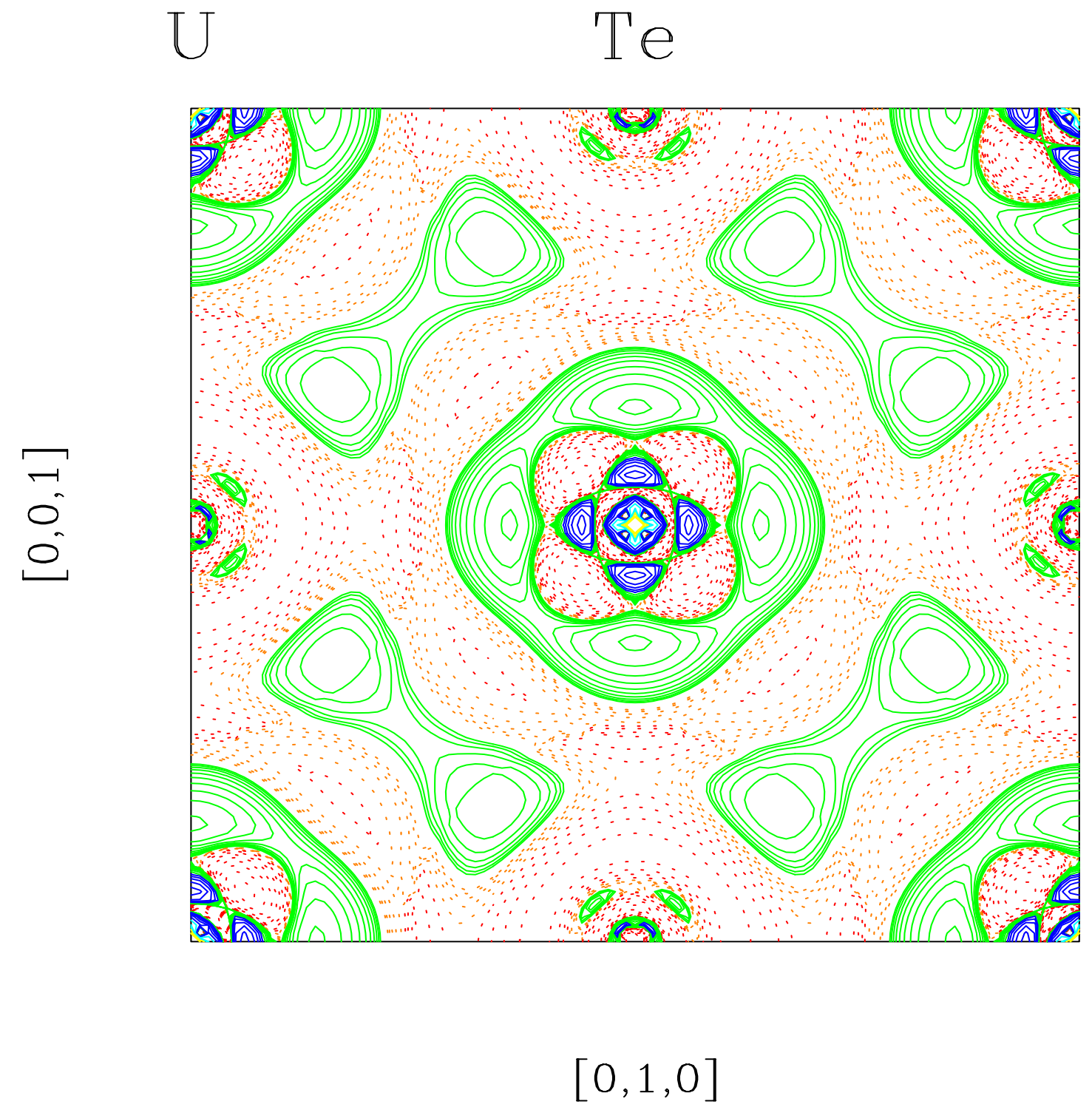


Figure 4.6: Uranium Phosphide bonding charge density, $\rho_{b o n d}$. The uranium atom sites are in the center and on the corners, the phosphorus sites are on the sides.

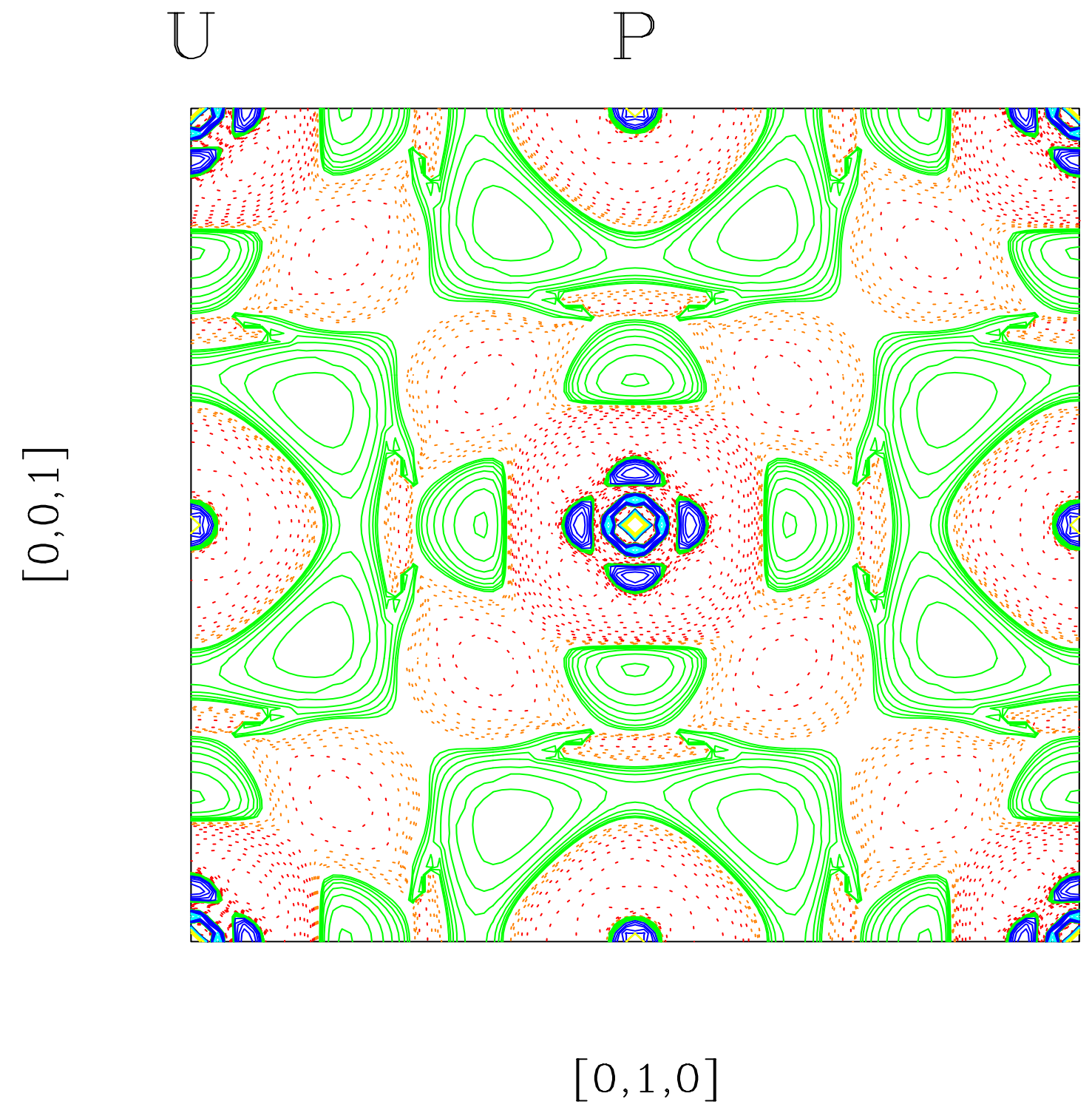


Table 4.7: Calculated (from the model hamiltonian method) and experimental values of the ordering temperatures and ordered moments of the uranium compounds. The ordering temperature listed is the Curie temperature for the chalcogenide compounds and the Néel temperature in the case of the pnictide compounds. Temperatures are listed in degrees Kelvin, ordered moments are given in $\mu_{B}$.

\begin{tabular}{|c|c|c|c|c|c|}
\hline & $T_{\text {ord }}$ calc & $T_{\text {ord }} \exp$ & & $\mu_{0}$ calc & $\mu_{0} \exp$ \\
\hline US & 950 & 178 & 3.1 & 1.6 \\
USe & 580 & 160 & 3.2 & 2.0 \\
UTe & 170 & 104 & 3.3 & 2.2 \\
\hline UP & 900 & 125 & 3.3 & 1.8 \\
UAs & 450 & 126 & 3.3 & 2.2 \\
USb & 195 & 241 & 3.3 & 2.8 \\
UBi & 120 & 285 & 3.2 & 3.0 \\
\hline
\end{tabular}

in qualitative agreement with experiment. We find that the calculated ordering temperatures for the more localized compounds (UBi, USb) are in better agreement with experiment, while overestimating $T_{\text {ord }}$ for the more itinerant compounds.

Notice that the moments obtained from the model hamiltonian method are all saturated, or nearly saturated. To explain this, we present the full 10x10 two ion interaction matrix for uranium sulfide, which, has the most delocalized $f$ states and behaves the most band like. This is demonstrated by the agreement between the pure electronic structure calculated results for US. The matrices for the first and second nearest neighbors are given in Table 4.8. At first glance, these tables seem a bit confusing, however, using insight gained for examination of cerium compound two-ion interaction matrices we can see why the moments obtained for the uranium compounds are saturated. First, notice that there is no sign change from first to second nearest neighbor terms for uranium sulfide. In the cerium chalcogenides, we had noted that this competition between ferromagnetic and antiferromagnetic inner plane couplings led to a reduced moment. Second, notice the dominance of the diagonal matrix elements of the two-ion interaction. In all 
cerium compounds, the addition of the crystal field term lowered the calculated ordered moments by introducing a mixing of $\mid \pm 5 / 2>$ with $\mp 3 / 2>$ states. This would show up in the final hamiltonian as off-diagonal elements. Here, we do see some appreciable off-diagonal elements likely due to the treatment of the $f$ electrons in the valence being affected by the crystal field. However, in order for the moment lowering effects to be noticed these terms would need to be on the order of the diagonal elements. In this particular case, we notice that these off-diagonal terms are about a factor of two too small to produce a lower moment. Therefore we can see why the final moments of these compounds would be saturated. This is a failure of this model to properly obtain the lowered moments which exist for the lighter uranium compounds.

Recall that the treatment of the $f$ state electrons placed into the valence to calculate the hybridization was designed to give a truer picture of how these $f$ electrons behave. We note that if these calculations were done from a "core" point of view, as the cerium compounds are, the ordering temperatures obtained would be an order of magnitude too small. ${ }^{10}$ Because of the failure of this calculation to obtain the correct moments for these compounds, it is very difficult to ascertain whether the treatment of the $f$ electrons gives the proper size of the hybridization or not. Using the Heisenberg model ${ }^{11}$, we find that the energy associated with the magnetic ordering temperature of the system is proportional to $J M^{2}$ where $J$ is the exchange integral and $M$ is the magnetic moment. To look at the size of the exchange parameters themselves, we now calculate an average scaled exchange integral for these systems by taking the ordering temperature and dividing it by the calculated ordered moment squared $\left(\mathcal{J}=T_{\text {ord }} / \mu_{0}^{2}\right)$. Next we compare these results to those calculated from the experimental values. These numbers are listed in Table 4.9. For this scaled parameter, good agreement between the model and experiment is obtained for the chalcogenide systems. This suggests that the primary reason for the poor agreement between the model and experiment in the calculation of the Curie temperatures stems from the overestimation of the ordered moment for these systems when using this model. For the pnictides, however, marginal agreement is only obtained for the larger systems and therefore we conclude that there must be an additional unknown factor in the failure of this model to calculate the ordering temperatures for the lighter 
Table 4.8: Total two-ion interaction matrices for the first and second nearest neighbors of US given in units of K.

\section{FIRST NEAREST NEIGHBOR}

$\left(\begin{array}{rrrrrrrrrr}338.1 & 5.9 & 2.0 & 20.7 & 40.7 & 37.6 & .0 & .0 & .0 & .0 \\ 5.9 & 305.4 & 6.0 & .7 & 7.5 & 41.4 & 100.3 & .0 & .0 & .0 \\ 2.0 & 6.0 & 266.2 & 18.4 & 1.3 & .7 & 8.5 & 131.6 & .0 & .0 \\ 20.7 & .7 & 18.4 & 222.5 & 47.5 & .3 & 3.1 & 8.5 & 100.3 & .0 \\ 40.7 & 7.5 & 1.3 & 47.5 & 175.0 & 66.7 & .3 & .7 & 41.4 & 37.6 \\ 37.6 & 41.4 & .7 & .3 & 66.7 & 175.0 & 47.5 & 1.3 & 7.5 & 40.7 \\ .0 & 100.3 & 8.5 & 3.1 & .3 & 47.5 & 222.5 & 18.4 & .7 & 20.7 \\ .0 & .0 & 131.6 & 8.5 & .7 & 1.3 & 18.4 & 266.2 & 6.0 & 2.0 \\ .0 & .0 & .0 & 100.3 & 41.4 & 7.5 & .7 & 6.0 & 305.4 & 5.9 \\ .0 & .0 & .0 & .0 & 37.6 & 40.7 & 20.7 & 2.0 & 5.9 & 338.1\end{array}\right)$

\section{SECOND NEAREST NEIGHBOR}

$\left(\begin{array}{rrrrrrrrrr}638.6 & 20.5 & 20.6 & 27.0 & 35.8 & 79.2 & .0 & .0 & .0 & .0 \\ 20.5 & 551.5 & 26.7 & 18.3 & 4.5 & 36.5 & 211.8 & .0 & .0 & .0 \\ 20.6 & 26.7 & 504.5 & 34.3 & 32.6 & 27.4 & 7.6 & 277.9 & .0 & .0 \\ 27.0 & 18.3 & 34.3 & 421.3 & 50.9 & 7.6 & 54.9 & 7.6 & 211.8 & .0 \\ 35.8 & 4.5 & 32.6 & 50.9 & 331.3 & 69.1 & 7.6 & 27.4 & 36.5 & 79.2 \\ 79.2 & 36.5 & 27.4 & 7.6 & 69.1 & 331.3 & 50.9 & 32.6 & 4.5 & 35.8 \\ .0 & 211.8 & 7.6 & 54.9 & 7.6 & 50.9 & 421.3 & 34.3 & 18.3 & 27.0 \\ .0 & .0 & 277.9 & 7.6 & 27.4 & 32.6 & 34.3 & 504.5 & 26.7 & 20.6 \\ .0 & .0 & .0 & 211.8 & 36.5 & 4.5 & 18.3 & 26.7 & 551.5 & 20.5 \\ .0 & .0 & .0 & .0 & 79.2 & 35.8 & 27.0 & 20.6 & 20.5 & 638.6\end{array}\right)$


pnictide systems.

We find that the model hamiltonian method provides good agreement with experimental values for the larger uranium systems. We also find the success of the "band point of view" treatment of the $f$ electrons when calculating the exchange parameters. However, this model fails to obtain good agreement for the lighter uranium systems obtaining saturated moments for these compounds as opposed to the lower moments due to the more itinerant nature of the $f$ electrons in these lighter systems.

Table 4.9: Average Scaled Exchange Parameters $\mathcal{J}$ calculated for both the results from the model hamiltonian method (MODL) and experiment (EXPT). These are listed in units of $K / \mu_{B}^{2}$.

\begin{tabular}{|c|c|c|c|c|c|c|c|c|}
\hline & US & USe & UTe & UP & UAs & USb & UBi \\
MODL & 99 & 57 & 16 & 82 & 41 & 18 & 12 \\
EXPT & 70 & 40 & 21 & 38 & 26 & 31 & 30 \\
\hline
\end{tabular}




\subsection{SUMMARY}

We have applied two different computational methods to study the trend of the lowtemperature ordered magnetic moment across the uranium compounds. The method involving pure electronic structure calculations predict better the moments for the more itinerant systems. On the other hand, the synthesis of phenomenology and electronic structure method provides a better description of the ordered moment for the more localized uranium compounds, and can be used to gain insight into some of the specific contributions of the interactions responsible for the unusual magnetic properties of these compounds. With a quite limited degree of success, the model hamiltonian calculations also give values for the magnetic ordering temperatures. We find that neither the FPLMTO spin polarized electronic structure calculations, nor the model hamiltonian calculations give a full picture across the spectrum of uranium compounds, and that each are limited to the range of the compounds most suited to the treatment of the $f$ electrons employed. The full band calculation involving spin and orbital polarization is most appropriate for the lighter more itinerant uranium systems where the $f$ electrons more freely form bands. The model hamiltonian, which in its derivation assumes distinct

felectron states, favors the heavier uranium compounds where such a distinction is more pronounced in an f-localized system. 


\subsection{FURTHER STUDIES}

Unlike the cerium compounds, where one of the methods employed found excellent agreement with experimental results for the whole range of those compounds, here we have used two methods which are limited to either end of the spectrum of uranium compounds. One for small uranium compounds, the other for the larger uranium systems. This has led to several studies and attempts to improve the methods to possibly capture the essence of the full scope of uranium compounds. The discussion of the methods explored are listed below. The symbol (MLDA) indicates those methods which are modifications to the pure LDA calculations whereas (MHMT) indicates those proposed modifications to the model hamiltonian method.

\subsubsection{LDA+U CALCULATIONS (MLDA)}

We noted earlier that the pure electronic structure FPLMTO calculations including orbital polarization were successful in calculating the ordered moments for the lighter uranium compounds, however, these results failed to show the nearly saturated moments for the heavier uranium systems. Therefore, we performed spin-polarized LDA+U FPLMTO calculations. ${ }^{12}$ This technique modifies the LDA energy functional by subtracting the LDA " $f-f$ " interactions, replacing them with on-site atomic-like interactions. The $U$ values used were those obtained $a b$ initio from the supercell calculations. However, the results of these calculations, even with no explicit orbital polarization, gave saturated moments for all compounds. Conversely, if we examine the ordered moment as a function of $U$ we find that for very small values (about $0.5 \mathrm{eV}$ ) the moment increases past the experimentally found value and for values of $U$ which are about $1.0 \mathrm{eV}$ begins to asymptotically approach saturation. These values are well below the calculated results

for the on-site Coulomb repulsion (3.4 to $4.8 \mathrm{ev}$ ) and thus this calculation gives virtually saturated moments for all uranium compounds. This calculation then is consistent with the model hamiltonian calculations. However, it fails to obtain the more band-like features of the lighter uranium compounds and is inappropriate for those systems. 


\subsubsection{CRYSTAL FIELD TERM (MHMT)}

Because of the success of the inclusion of the crystal field splitting term in the cerium compound hamiltonian, we examine a possible inclusion of such a term in the hamiltonian for the uranium compounds. However, because of several technical problems and questions which enter into the formalism of this method, we have not, to date, carried out such computations. Here, we discuss the difficulties.

First, the crystal field splitting of the uranium systems is much more complex than that of the cerium compounds. Recall that the magnetic ordering of the uranium systems is dictated by $f^{3}$ multiplets. We would then have to incorporate the crystal field effects on the $j=9 / 2$ manifold of these systems. In terms of the Stevens equivalence operators used earlier we now have

$$
H=B_{4}\left(O_{4}^{0}+5 * O_{4}^{4}\right)+B_{6}\left(O_{6}^{0}-21 * O_{6}^{4}\right)
$$

Notice for the $j=9 / 2$ manifold we must include $\left\langle r^{6}>\right.$ terms which are given in reference 13. The $j=9 / 2$ manifold is split into a two fold degenerate $\Gamma_{6}$ and two four fold degenerate $\Gamma_{\mathbf{8}}^{(1)}$ and $\Gamma_{\mathbf{8}}^{(2)}$ states. Depending upon the ratio of $B_{4} / B_{6}$ different ground states would be found for the crystal field. In this crystal field, mixing occurs between $| \pm 9 / 2>,| \pm 1 / 2>$, and $\mid \mp 7 / 2>$ states; as well as between $\mid \pm 5 / 2>$ and $\mid \mp 3 / 2>$. Thus, it is conceivable that the addition of such a term could lower the calculated moments in the model hamiltonian method. However, the problems with such an approach begin to become visible when we attempt to chose the parameters $B_{4}$ and $B_{6}$. First, we have no $a b$ initio calculations of the crystal field splitting for these compounds. Even with such a calculation of the total crystal field splitting there would not necessarily be any indication as to the size of the individual parameters involved. In order to perform a calculation in the two parameter space in question $\left(B_{4}\right.$ and $\left.B_{6}\right)$ one needs to calculate each parameter from first principles or at least obtain the ratio of the parameters from first principles, then use the total crystal field splitting. Next, we are absent experimental results for the crystal field splittings for most of the uranium compounds. Only those for UTe and USb are currently available.

Another difficulty occurs in the treatment of the $5 f$ electrons in the band calculation. 
Recall that unlike the cerium compounds, when using the model hamiltonian method we place the uranium $5 f$ electrons into the valence band when performing the FPLMTO calculation of the model parameters. Therefore, these two-ion interactions already have some crystal field effects included. To then include a separate crystal field term would be double counting such effects. If one wishes to include the crystal field in the model hamiltonian, as we do with the cerium compounds, one must not allow the crystal field effects to enter into the other parameters. This could be accomplished via the "core" point of view, (ie. placing the $5 f$ electrons in the core as we do for the cerium compounds). However, as we discussed earlier it is inappropriate to treat the $5 f$ state electrons of uranium in this way. A better solution would be to use a warped muffin-tin calculation of the parameters entering into the model hamiltonian. The $f$ states would still be allowed the self-banding property but will only be affected by a spherically averaged potential, since most of the $f$ state resides inside the muffin-tin sphere. Even this approach, though, has its drawbacks. In the warped muffin-tin calculation not only are the crystal field effects not included for the $f$ electrons, but it also neglects the crystal field effects on the non- $f$ electrons.

Further pursuit of this type of calculation is therefore left for future work.

\subsubsection{CHANGING THE MODEL}

The two methods employed to calculate the magnetic properties of the uranium compounds are limited in application. One approach is to make modifications to these models, whereas the other is in the discarding of the model itself. The problem with the uranium compounds is the wide range of $f$ electron localization which varies from being nearly localized to almost band like. Neither of the methods used in this calculation allow for such a dynamic in the $f$ electron behavior. Either we have tailored our picture to work well with the more localized compounds, or we have used a view which favors

the more band like $f$ states. In order to obtain good agreement with experiment for the whole range of compounds, we need to adopt a model which would allow for both types of $f$ electrons to enter into the picture. In such a model the degree of $f$ localization could be accounted for by two different types of $f$ states, one more band like, the other atomic 
like. Employing an $a b$ initio based phenomenological approach utilizing the Hubbard model and a disordered Ising lattice with randomly-located holes (two types of uranium sites, embodying the localized and delocalized components, respectively) Cooper and $\operatorname{Lin}^{11}$ obtain the correct values of ordering temperatures and variation with pressure and dilution alloying for the uranium chalcogenides. These results capture the whole range of the uranium chalcogenide systems as opposed to just one specific type of uranium compound. 


\section{References}

1. H. Rudiger, H. R. Ott, and O. Vogt, Physica B 130, 538 (1985).

2. B. Reihl, N. Martensson, and O. Vogt, J. Appl. Phys. 53, 2008 (1982).

3. S. K. Sinha, G. H. Lander, S. M. Shapiro, and O. Vogt, Phys. Rev. 23, 4556 (1981).

4. T. M. Holden, W. J. L. Buyers, E. C. Svensson, J. A. Jackman, A. F. Murray, O. Vogt, and P. de. V. DuPlessis, J. Appl. Phys. 53, 1967 (1982).

5. T. M. Holden, W. J. L. Buyers, P. de. V. DuPlessis, K. M. Hughes, and M. F. Collins, J. Magn. Magn. Mater. 54-57, 1175 (1986).

6. O. Eriksson, M. S. S. Brooks, and B. Johansson, Phys. Rev. B 41, 7311 (1990).

7. D. L. Price and B. R. Cooper, Phys. Rev. B 39, 4945 (1989).

8. Q. G. Sheng and B. R. Cooper, J. Appl. Phys. 69, 5472 (1991); Phys. Rev. B 50,965 (1994).

9. N. Kioussis, B. R. Cooper, and J. M. Wills, Phys. Rev. B 44, 10,003 (1991).

10. Q. G. Sheng, B. R. Cooper, and S. P. Lim, Phys. Rev. B 50, 9215 (1994).

11. C. Kittel, Introduction to Solid State Physics 7th edition (John Wiley \& Sons, Inc. 1996).

12. D. L. Price, B. R. Cooper, S. P. Lim, and I. Avgin, Submitted to Phys. Rev. B.

13. K. R. Lea, M. J. M. Leask, and W. P. Wolf, J. Phys. Chem. Solids 23, 1381 (1962).

14. B. R. Cooper, O. Vogt, Q. G. Sheng, and Y. L. Lin, Phil. Mag. B 79, 683 (1999). 


\section{Chapter 5}

\section{CONCLUSIONS}

We have successfully carried out two $a b$ initio based calculations of the magnetic properties of both cerium and uranium compounds.

We find that the pure band calculations, even with the full-potential and orbital polarization correction, fail to even qualitatively obtain agreement with experiment for the cerium compound systems. For the uranium compounds, this method involving a purely band treatment of the $f$ state electrons is confined in its success to the lightest uranium systems (US and UP), and fails to predict the more saturated moments of the larger uranium compounds. We conclude that these LDA calculations are inappropriate for the correlated $f$ electrons in these classes of compounds. We find that the larger the degree of localization of the $f$ electrons in these systems, the larger the discrepancy between the calculated moments using this band approach and experimental results.

The model hamiltonian approach, when including both hybridization and Coulomb exchange parameters on an equal footing, in the case of the uranium systems, provides better agreement for the heavier uranium systems. However, this method fails to predict anything aside from saturation for the ordered moments of these compounds, and therefore does not show the decreasing moment as the uranium-uranium spacing decreases for the lighter uranium compounds found in experiment. Primarily due to this overestimation of the ordered moments, the ordering temperatures for the lighter compounds are likewise much larger than experiment. Since we have seen that the purely band structure calculations succeed only at the other extreme of the uranium $f$ electron 
localization-delocalization, we conclude that what is needed is a model which can accommodate both extremes of the electron localization found in the uranium monopnictide and monochalcogenide systems. Such a model would take into account the self-banding of the uranium compound $5 f$ states pronounced in the lighter uranium compounds, while at the same time incorporate the important correlation effects prevalent in the heavier uranium systems.

For the cerium compound studies, we have found the model hamiltonian calculation with the inclusion of hybridization exchange, Coulomb exchange, and crystal field splitting all on an equal basis, give results for the ordered moment and ordering temperature which are in excellent agreement with the experimental values across the whole series of these compounds. The success includes predicting the large moment suppression from $\mathrm{CeSb}$ to CeTe whereas the only difference in these two compounds chemically is the addition of a $p$ electron. These results demonstrate the importance of the crystal field effects as well as the interplay between the three pertinent interactions (hybridization, Coulomb exchange, and crystal field) in determining the magnetic properties of the cerium monopnictides and monochalcogenides. The large degree of the success of this calculation even allowed for the examination of some of the individual configurations for the cerium pnictide systems, and provided valuable insight into which kinds of interactions favor different configurations. The problem of understanding the cerium compound systems on a fully $a b$ initio basis has been reduced to the remaining calculation of the crystal field splitting term strictly from first principles. 